University of New Hampshire

University of New Hampshire Scholars' Repository

Fall 2011

\title{
Experimental investigation of helical cross-flow axis hydrokinetic turbines, including effects of waves and turbulence
}

Peter Bachant

University of New Hampshire, Durham

Follow this and additional works at: https://scholars.unh.edu/thesis

\section{Recommended Citation}

Bachant, Peter, "Experimental investigation of helical cross-flow axis hydrokinetic turbines, including effects of waves and turbulence" (2011). Master's Theses and Capstones. 649.

https://scholars.unh.edu/thesis/649

This Thesis is brought to you for free and open access by the Student Scholarship at University of New Hampshire Scholars' Repository. It has been accepted for inclusion in Master's Theses and Capstones by an authorized administrator of University of New Hampshire Scholars' Repository. For more information, please contact Scholarly.Communication@unh.edu. 


\section{EXPERIMENTAL INVESTIGATION OF HELICAL CROSS-FLOW AXIS HYDROKINETIC TURBINES, INCLUDING EFFECTS OF WAVES AND TURBULENCE}

BY

PETER BACHANT

BSME, University of Massachusetts Dartmouth, 2008

THESIS

Submitted to the University of New Hampshire

in Partial Fulfillment of

the Requirements for the Degree of

Master of Science

in

Mechanical Engineering

September, 2011 
UMI Number: 1504939

All rights reserved

INFORMATION TO ALL USERS

The quality of this reproduction is dependent upon the quality of the copy submitted.

In the unlikely event that the author did not send a complete manuscript and there are missing pages, these will be noted. Also, if material had to be removed, a note will indicate the deletion.

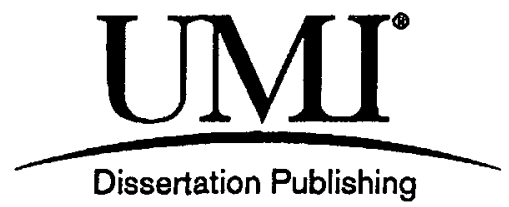

UMI 1504939

Copyright 2011 by ProQuest LLC.

All rights reserved. This edition of the work is protected against unauthorized copying under Title 17, United States Code.

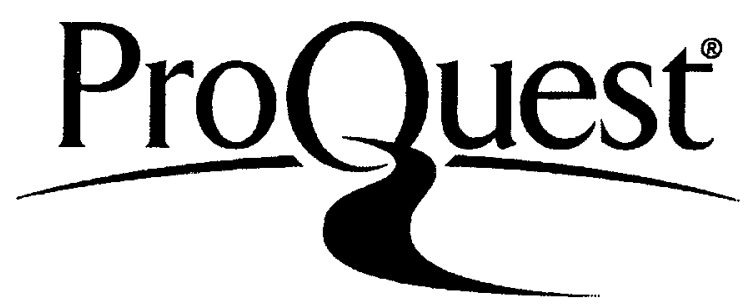

ProQuest LLC

789 East Eisenhower Parkway

P.O. Box 1346

Ann Arbor, MI 48106-1346 
This thesis has been examined and approved.

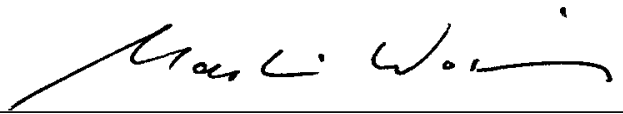

Thesis Director, Martin M. Wosnik, Assistant Professor Mechanical Engineering

Kenteth C. Baldwin, Professor of Mechanical Engineering and Ocean Engineering and Marine Sciences<smiles>C1CCCCCC1</smiles><smiles>CC(C)CC(C)CC(C)CC(C)C</smiles>
Josepb C/Klewicki, Professor of Mechanical Engineering

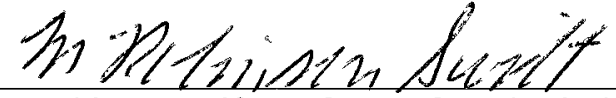

M. Robinson Swift, Professor of Mechanical Engineering and Ocean Engineering

Date




\section{Acknowledgments}

The author would like to acknowledge the contributions of all who supported this research, financially or otherwise.

Thanks to Lucid Energy Technologies, LLP. Thanks to the UNH Vice Provost for Research and the Dean of the College of Engineering and Physical Sciences for RA support. Thanks to the UNH ME department for TA support. Thanks to the NE-MREC for their Summer Fellowship.

Thanks to Alex Johnston, Ivaylo Nedyalkov, and Paul Lavoie for help with fabrication and assembly of the test bed. Thanks to Ivaylo Nedyalkov and Matt Rowell for their riding onboard the carriage acting as the turbine brake actuator. 


\section{Table of Contents}

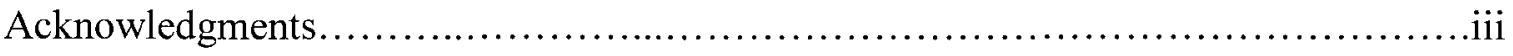

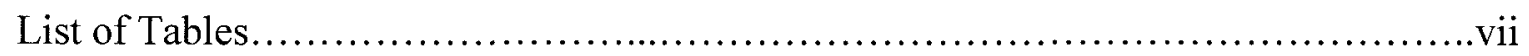

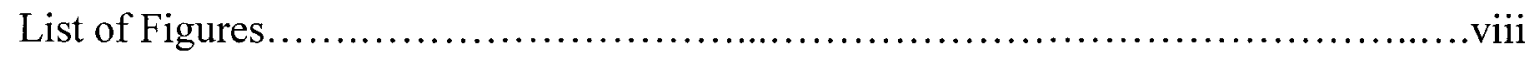

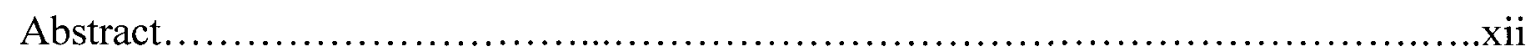

$\begin{array}{ll}\text { Chapter } & \text { Page }\end{array}$

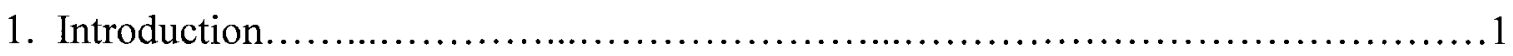

1.1 Objectives...........................................................

2. Cross-Flow Axis Hydrokinetic Turbines: A Theoretical Overview....................10

2.1 Capturing Fluid Kinetic Energy - Single Actuator Disk Theory...............10

2.2 Blockage Effects and Correction..................................... 15

2.3 Flow and Forces on a Turbine Blade.................................... 18

2.4 Foil Performance in Turbulence............................................24

2.5 Rotating Rigid Body Dynamics.......................................... 25

2.6 Cross-Flow Axis Turbine Design Parameters..............................26

2.7 Linear Progressive Surface Wave Theory ..................................26

3. Development of a Hydrokinetic Turbine Test Bed for the UNH Tow \& Wave Tank..28

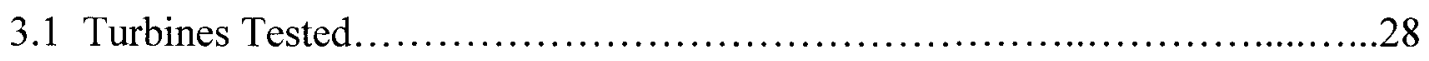

3.2 The UNH Tow \& Wave Tank.......................................... 31

3.3 Test Bed Design: Desired Characteristics and Concept Selection..............32

3.4 Main Frame Assembly: Turbine Mounting, Loading, and RPM

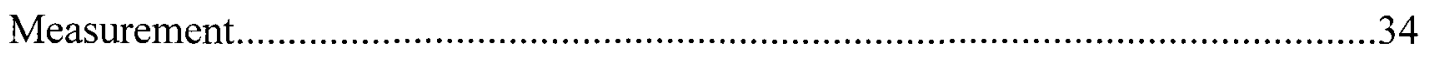


3.5 Drag Measurement - Rail Subassemblies...................................40

3.6 Signal Conditioning and Data Acquisition.........................................41

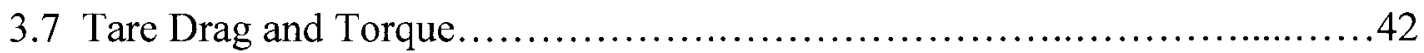

3.8 Final Test Bed Design Summary .........................................44

3.9 Add-ons: Turbulence Generators..........................................47

3.10 Add-ons: Flow Measurement Devices........................................49

4. Turbine Performance in Steady Uniform Flow.....................................51

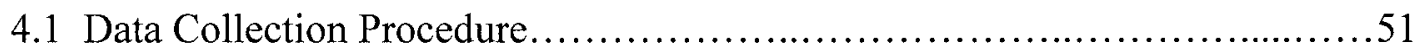

4.2 Data Reduction and Uncertainty Estimates..............................52

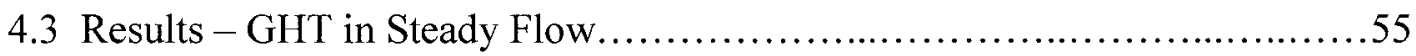

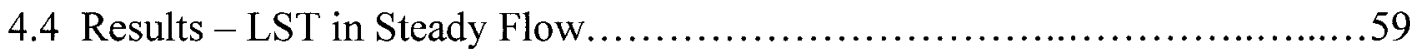

4.5 Tip Speed Ratio Limitations in Passive Loading Configuration..................64

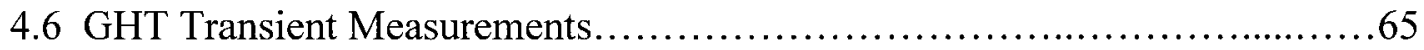

4.7 Conclusions - Steady Performance........................................6

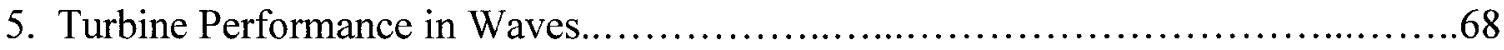

5.1 Results - GHT Performance in Waves.................................. 70

5.2 Developing a 2D Model to Explain Results.................................73

5.3 Conclusions - Performance in Waves...................................... 81

6. Turbine Performance in Turbulence.............................................. 82

6.1 Delay of Static Stall - PIV Measurements..................................8

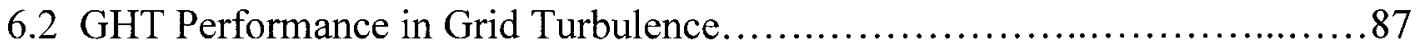

6.3 GHT Performance in Turbulent Cylinder Wake...............................99

6.4 Conclusions - Performance in Turbulence.................................96 


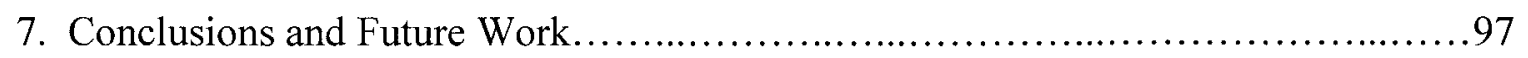

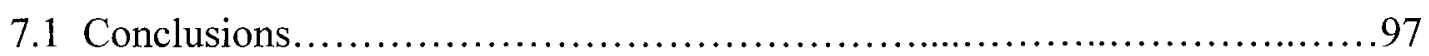

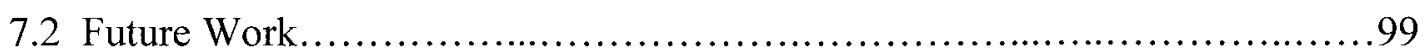

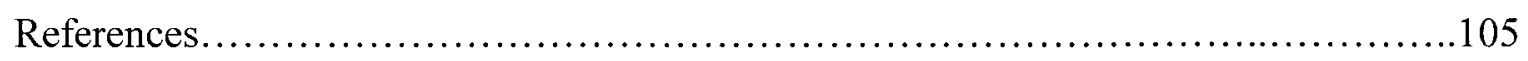

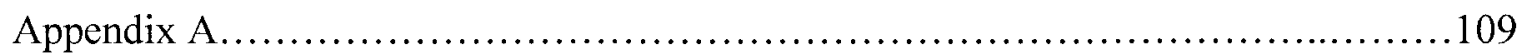

A.1 Tips for Test Bed Operation and Lessons Learned............................109

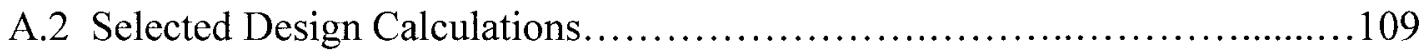

A.3 Additional Instrumentation and Component Details........................111

Appendix B.................................................................. 118

B.1 Suggestions for Tow Tank Improvement..................................118

B.2 Suggestions for Hydrokinetic Turbine Test Bed Improvement.................123 


\section{List of Tables}

Table

Page

3.1 Turbine specifications...................................................

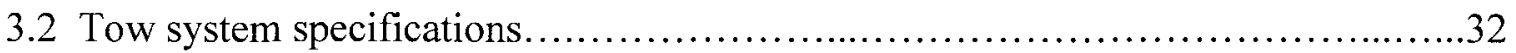

3.3 Turbine test bed characteristics as built....................................46

4.1 Summary of experimental error estimates.......................................54

5.1 Theoretical wave parameters averaged over turbine height...................... 70

6.1 Cylinder flow parameters....................................................

A.1 Individual component error considered................................... 111

A.2 Part numbers for selected purchased items.................................... 112 


\section{List of Figures}

Figure

Page

1.1 Drawings from Darrieus' patent...............................................

1.2 Drawing from Gorlov' helical turbine patent.................................... 6

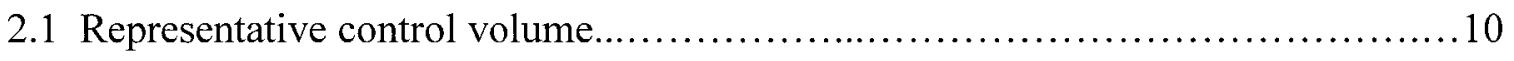

2.2 Turbine control volume in a blocked flow................................... 16

2.3 Blade geometry and force diagrams for unstalled and stalled conditions.............19

2.4 Theoretical turbine blade angle of attack at various tip speed ratios.................22

2.5 Theoretical relative velocity seen by a turbine blade throughout one rotation........22

2.6 Lift to drag ratio versus angle of attack for various airfoils $\ldots \ldots \ldots \ldots \ldots \ldots \ldots \ldots . . .23$

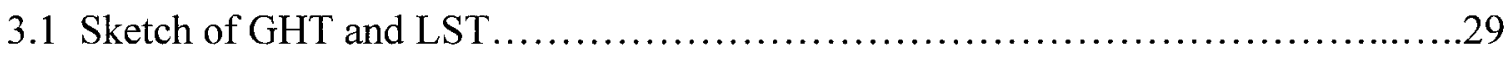

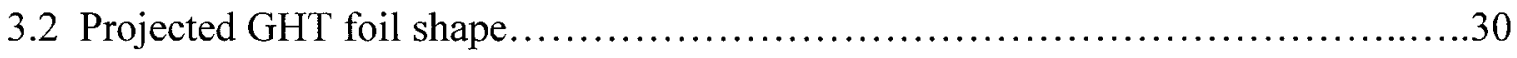

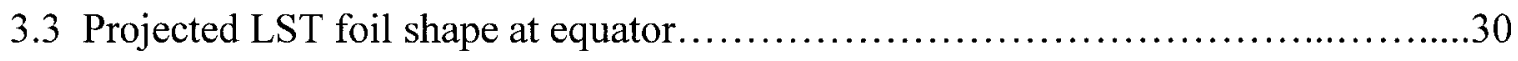

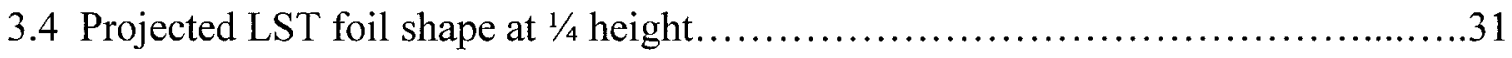

3.5 Projected LST foil shape near poles........................................ 31

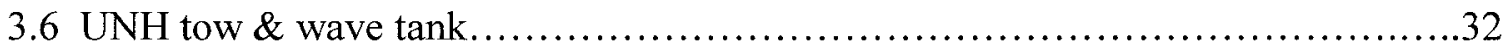

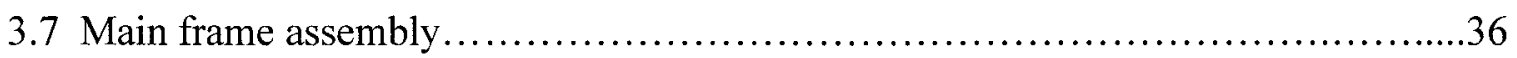

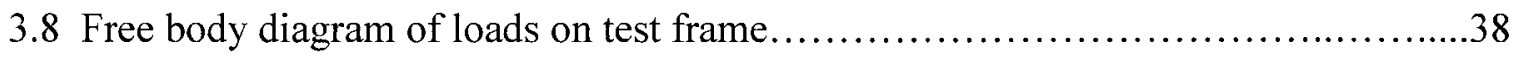

3.9 Exploded view of brake lever bracket assembly............................. 39

3.10 Rail subassembly ............................................................ 40

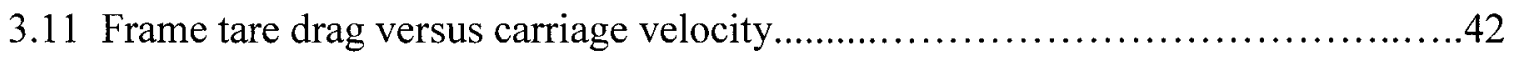

3.12 Tare torque versus RPM for forward and reverse directions.....................43

3.13 UNH-CORE hydrokinetic turbine test bed outline.........................................45 
3.14 GHT and LST installed in UNH-CORE hydrokinetic turbine test bed. .46

3.15 Grid turbulence generator. 48

3.16 Cylinder wake turbulence generator.

4.1 Sample data collection time series for one tow. 52

4.2 GHT power coefficient versus tip speed ratio without blockage correction. .56

4.3 Blockage-corrected GHT power coefficient versus blockage-corrected tip speed ratio. .56

4.4 GHT drag coefficient versus tip speed ratio without blockage correction.............58

4.5 Blockage-corrected GHT drag coefficient versus blockage-corrected tip speed ratio .58

4.6 GHT kinetic exergy efficiency versus tip speed ratio.

4.7 LST power coefficient versus tip speed ratio without blockage correction

4.8 Blockage-corrected LST power coefficient versus blockage-corrected tip speed ratio. .61

4.9 LST drag coefficient versus tip speed ratio without blockage correction .62

4.10 Blockage-corrected LST drag coefficient versus blockage-corrected tip speed ratio

4.11 LST kinetic exergy efficiency versus tip speed ratio

4.12 GHT torque coefficient versus tip speed ratio .64

4.13 GHT steady and transient power coefficients versus tip speed ratio. .66

5.1 Sketch of GHT operating under a wave in the tow tank. .69

5.2 Theoretical wave velocity and displacement amplitudes versus depth .69

5.3 Sample data collection run in $2.5 \mathrm{~s}$ period waves. .71 
5.4 GHT power coefficient versus tip speed ratio with and without waves present........72

5.5 GHT drag coefficient versus tip speed ratio with and without waves present...........72

5.6 Model power coefficient for various constant induction factors and experimental results plotted versus tip speed ratio

5.7 Power coefficient versus tip speed ratio for the steady model, experiments, and results from $1 \mathrm{D}$ momentum theory

5.8 Drag coefficient versus tip speed ratio for the steady model, experiments, and results from $1 \mathrm{D}$ momentum theory .76

5.9 Lift to drag ratio for one half rotation for two tip speed ratios. .77

5.10 Spectrum of model output torque for various tip speed ratios without waves present .78

5.11 Power coefficient versus tip speed ratio calculated for six rotations with and without $1.5 \mathrm{~s}$ period waves present .79

5.12 Torque spectral density predicted by the model under a $2.5 \mathrm{~s}$ period wave. .80

6.1 Lift and drag coefficients for NACA 0021 in various levels of grid turbulence. .84

6.2 Lift to drag ratio for NACA 0012 foil at various Reynolds numbers. .86

6.3 Delay of stall experimental setup. .86

6.4 Instantaneous velocity vectors and vorticity contours for NACA 0012 hydrofoil with and without grid turbulence .86

6.5 Mean velocity contours normalized by free stream velocity with and without grid turbulence .87

6.6 UNH-CORE hydrokinetic turbine test bed with grid turbulence generator installed. .88 
6.7 GHT power coefficient versus tip speed ratio in grid turbulence.

6.8 GHT drag coefficient versus tip speed ratio in grid turbulence.

6.9 UNH-CORE hydrokinetic turbine test bed with cylinder wake turbulence generator

installed.

6.10 Schematic showing cylinder wake experimental setup

6.11 GHT power coefficient in 4.5 inch diameter cylinder wake at various cross-stream

locations

6.11 GHT drag coefficient in 4.5 inch diameter cylinder wake at various cross-stream locations

A.1 Cross-section of lower horizontal foil member.

A.2 Factory calibration sheets for load cells

A.3 Factory calibration sheet for load cell signal conditioners.

A.4 Factory calibration sheet for RPM frequency signal conditioner

A.5 Factory calibration sheet for carriage speed signal conditioner.

A.6 Factory calibration sheet for torque transducer

B.1 Simplified model of tow carriage system

B.2 Tow carriage velocity during acceleration for the model and measurements. 


\begin{abstract}
EXPERIMENTAL INVESTIGATION OF HELICAL CROSS-FLOW AXIS

HYDROKINETIC TURBINES, INCLUDING EFFECTS OF WAVES AND

TURBULENCE

by

Peter Bachant

University of New Hampshire, September, 2011
\end{abstract}

A test bed for cross-flow axis (CFA) hydrokinetic turbines for the UNH tow and wave tank was designed and built, and two different turbines were evaluated. The effects of waves and turbulence on performance were also observed. Drag (thrust) and mechanical power output were measured at tow speeds of $0.6-1.5 \mathrm{~m} / \mathrm{s}$ for a Gorlov Helical Turbine (GHT) and a Lucid Spherical (helical) Turbine (LST), both provided by Lucid Energy Technologies, LLP. Overall, the GHT performed with a higher power and drag coefficient than the LST. GHT performance was enhanced by progressive waves, but waves caused stalling at higher tip speed ratios compared to the steady case. Grid turbulence enabled the GHT to operate at lower tip speed ratios and did not alter maximum power coefficient, but increased drag on the turbine. Performance in a cylinder wake was highly dependent on the cylinder's cross-stream location, ranging from benign to detrimental. 


\section{Introduction}

Industrial and human development, often measured in terms of life expectancy, literacy or per-capita income - are closely coupled to the availability and use of energy in a society. Presently, useful energy is derived mainly from fossil fuels such as coal and oil, which are essentially solar energy that has been stored in the Earth's crust over very long timescales (McKay, 2009). Unfortunately, the rate of fossil fuel use far outpaces their rate of natural creation (McKay, 2009). Therefore, to sustain human development and the technical complexity of our society beyond the depletion of fossil fuels, we must develop ways to capture more of the solar power incident on the Earth, i.e. renewable energy. Improved renewable energy conversion technology will improve quality of life for humans on longer timescales than continuing to predominantly rely on non-renewable energy sources.

Hydrokinetic turbines are devices that can remove energy from moving water, which is found in river flows, tidal and ocean currents, as well as in waves. These sources of energy are considered renewable, and can mostly be attributed to the Earth's moon and Sun. Solar energy is converted into kinetic energy in river flows through the water cycle, whereas the kinetic energy in tidal flows is the result of gravitational forcing from celestial bodies, mainly the Earth's moon, and to a lesser degree the Sun. This forcing does work on the oceans of the Earth, creating flows of water, whose energy is dissipated through viscous dissipation at the seabed (bottom friction) and turbulence as a result of moving water through natural restrictions and around coast lines. This in turn dissipates energy from the astronomical system. However, the Earth's loss of rotational energy is 
only noticeable over millions of years (Williams, 2000), meaning the kinetic energy within these flows can be extracted with negligible effects on the astronomical dynamics. It is estimated that hydrokinetic power sources, namely waves, tides, ocean currents, and river flows have the potential to provide $10 \%$ of the total power consumption of the world (Electric Power Research Institute, 2006) (World Energy Council, 2007).

The use of hydrokinetic power began with early waterwheels, which were invented by the Greeks in the Hellenistic period and extensively used by the Greeks and Romans for milling (Donners, Waelkens, \& Deckers, 2002). Increasingly, dams and impoundments were used to increase the available net head, and hydropower developed into the conventional hydroelectric stations we know today. Conventional hydropower is presently the largest renewable energy source in the world with approximately a $16 \%$ share of global electric energy production (International Energy Agency, 2010), and about $6 \%$ in the United States (United States Department of Energy, 2010). While a conventional hydropower installation can extract the available energy more efficiently than a hydrokinetic system, the environmental effects due to the massive dams, flooded areas and changes in sediment and nutrient transport processes are much more pronounced, and can be ecologically detrimental. Large hydro turbines (w/ Francis, Kaplan or Pelton runners) can achieve energy conversion efficiencies of greater than $90 \%$ (Arndt, 1991), whereas efficiencies of sparsely deployed hydrokinetic turbines are limited to $59.3 \%$ by Betz' law, and in practice are approaching $50 \%$ for the largest hydrokinetic installations, similar to large wind turbines.

Commercial development of tidal energy is not new. Small barrage scheme tidal "mills" with conventional water wheels were used in Southern England and Northern 
France in the Middle Ages, as well as in New England (e.g., Boston Mill Pond) through the $1800 \mathrm{~s}$. In the latter half of the $20^{\text {th }}$ century (in particular after 1973 oil crisis), tides and waves have been seriously re-examined as potential sources of (electric) energy. A 240 MW tidal-barrage power plant has been in operation at La Rance in Brittany, France since 1966 (Banal \& Bichon, 1981). However, similar to conventional hydroelectric installations, large-scale barrages have the downside that they significantly alter ecosystems and are very costly to build.

Cross-flow axis (CFA) turbines are defined as having their axis of rotation perpendicular to the incoming flow direction. They differ from in-stream axis turbines in that they rotate the same direction regardless of inflow direction, negating the need for yaw control to turn the rotors into the flow. This decrease in system complexity comes with more complicated, and arguably more interesting fluid dynamics. The first fully submerged cross-flow axis turbine was likely a Savonius rotor, a design that was originally intended for wind power, patented in 1930 (Savonius, 1930). It is a simple drag driven device that resembles two halves of a barrel reattached with an offset of one diameter (sometimes slightly less). Savonius rotors are still being used today despite their relatively lower efficiencies compared with other designs. They are considered very robust and will start to rotate as soon as fluid dynamic drag force (moment) exerted on the rotor exceeds the bearing friction force (moment), resulting in lower "cut in speeds." The cut in speed is defined as the wind or water velocity at which the rotor begins turning, allowing energy extraction. In the 1930s, Darrieus invented a CFA turbine that replaced the mostly drag driven "buckets" of the Savonius rotor with mostly lift driven foils to increase conversion efficiency by allowing the blade tangential velocity to exceed 
the free stream velocity (Darrieus, 1931). Excerpts of Darrieus' patent are shown in Figure 1.1.

Darrieus devices were explored extensively for wind energy applications during the 1980 s into the $1990 \mathrm{~s}$, leading to the deployment of a $34 \mathrm{~m}$ wind turbine by Sandia National Laboratories in collaboration with the US Department of Energy (DOE), and culminating with the installation of the world's largest vertical axis wind turbine, Eole, rated at $4 \mathrm{MW}$, at Cap Chat in Quebec, Canada in 1985 (Paraschivoiu, 2002). Despite their respectable power outputs, development of these straight-bladed (with respect to azimuth location) cross-flow axis turbines was essentially abandoned, since the large variations in torque, due to large variations in lift as the blade angle of attack varies throughout the rotation, caused structural fatigue failures of the turbines. 


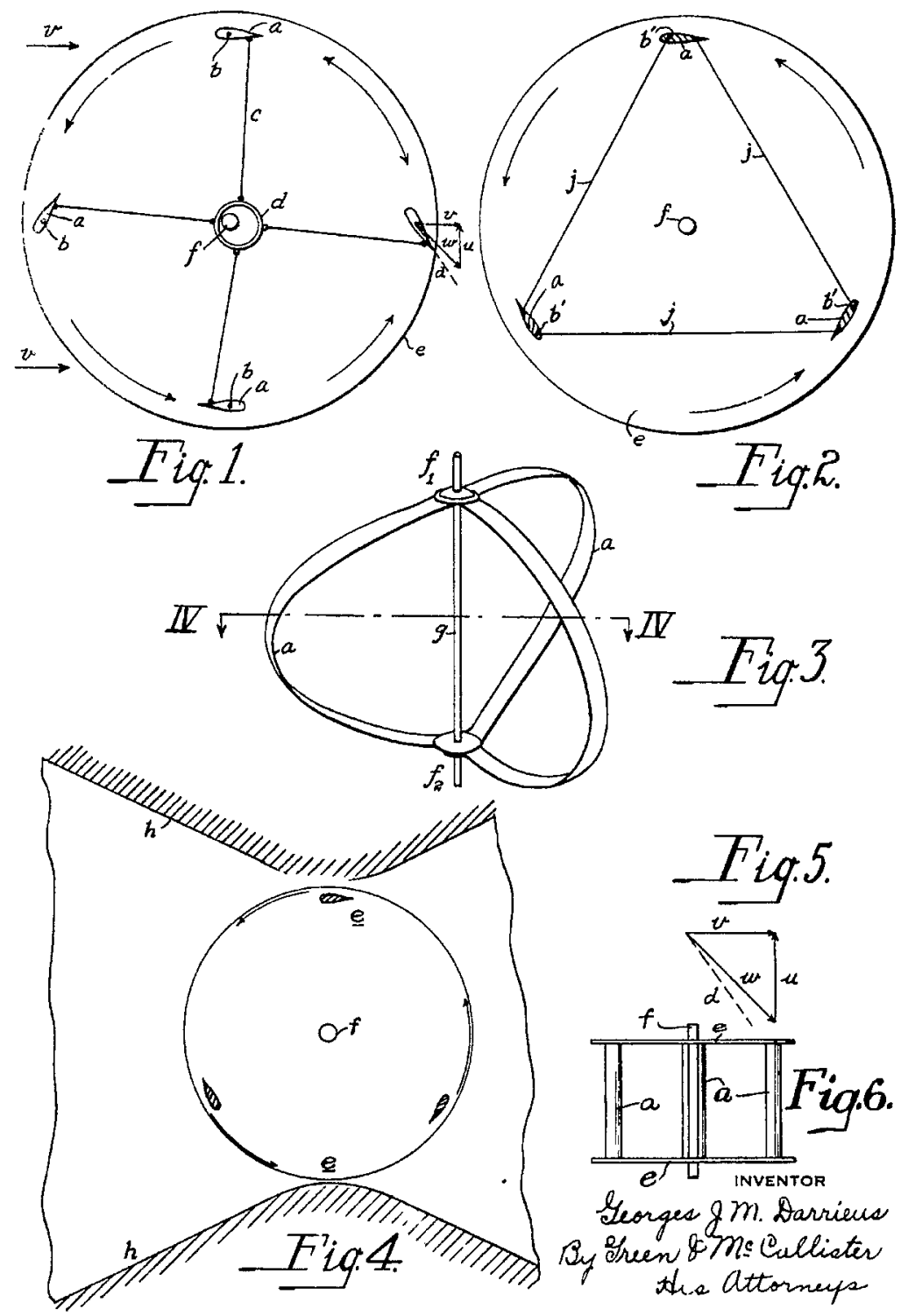

Figure 1.1 Drawings from Darrieus’ patent (Darrieus, 1931).

In 1995 Alexander Gorlov designed a cross-flow axis turbine with its blades swept helically to help average the periodically unsteady torques inherent in the straight-bladed concept (Gorlov, 1995). The swept blades also made the device more prone to selfstarting, which for a Darrieus is highly dependent on the number of blades, initial rotor position, etc. Gorlov Helical Turbines (GHTs) are practically identical in two dimensions 
compared to their straight-bladed counterparts. Consequently, Darrieus turbine performance models and experimental observations are relevant.

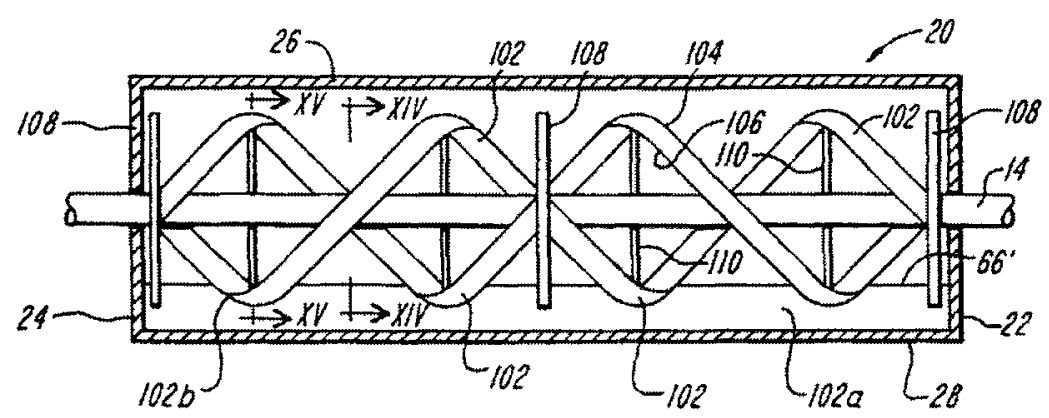

Figure 1.2 Drawing from Gorlov's helical turbine patent (Gorlov, 1995).

To date, there is a scarcity of performance data for helical devices in the literature. The limited information available shows Gorlov Helical Turbines to reach efficiencies as high as 35\% (Gorlov, Development of the Helical Reaction Hydraulic Turbine, 1998). Peak efficiencies for smaller straight-bladed Darrieus turbines in water have been reported around 23\% (Shiono, Suzuki, \& Kiho, 2000), whereas the peak efficiencies of a $17 \mathrm{~m}$ diameter Darrieus wind turbine at Sandia National Laboratory were reported to exceed 40\% (Paraschivoiu, 2002).

Lucid Energy Technologies, LLP (Lucid), formerly GCK Technologies (where "G" stood for Gorlov), carried on the development of the GHT, and succeeded with several small-scale installations over the years. Lucid/GCK also provided two prototypes to the Korean Ocean Research and Development Institute (KORDI) for testing in the Uldolmok Tidal Strait (GCK Technologies, 2002-2006). Based on the experience with the Lucid/GCK GHTs, in 2009, KORDI completed their Uldolmok Tidal Current Power Plant, a full scale test project rated at $1 \mathrm{MW}$. The $6.5 \mathrm{~m} / \mathrm{s}$ maximum currents are 
harnessed via two vertical axis helical devices (Lee, Yum, Park, \& Park, 2009). In the United States, the Ocean Renewable Power Company (ORPC) is using Gorlov Helical Turbines arranged in pairs on either side of a common generator with horizontal axis in a demonstration installation in Eastport, ME (Ocean Renewable Power Company, 2008) (Testing the Waters with Tidal Energy, 2010).

Recently, Lucid developed vertical axis spherical-helical turbines to be installed in pipe sections, with the goal to harvest excess energy available in large gravity-fed water pipes, for example in irrigation or wastewater systems. The drop-in installation has been given the name Northwest PowerPipe ${ }^{\mathrm{TM}}$ (Schlabach, 2010). Since these turbines are installed in a high blockage closed conduit, they are similar to traditional hydropower installations and the efficiency limit for turbines installed in a free stream, also known as the "Betz limit," does not apply.

The UNH Center for Ocean Renewable Energy (UNH-CORE) was founded in 2008 as a collaborative environment for academic research and commercial development of marine renewable energy devices. Starting in late 2008 through 2010, TECH 797 senior design project groups working with UNH-CORE faculty deployed two sizes of Gorlov Helical Turbines from a $35 \mathrm{ft} \times 10 \mathrm{ft}$ test platform at the UNH-CORE Tidal Energy Test Site in the Great Bay Estuary in New Hampshire.

This thesis describes the first steps of a more thorough experimental investigation of helical cross-flow axis hydrokinetic turbines. Regarding practical value, if turbine performance is optimized, more energy is captured. If peak performance operating parameters are known (namely tip speed ratio, defined in Chapter 2), turbine control is a matter of keeping the turbine rotating at a tip speed ratio where efficiency is maximized. 
This could be approached iteratively with closed loop feedback control, or it could be prescribed based on measurements of the environment the device is placed in; namely mean flow speed and direction, turbulence characteristics, and wave action. It's possible that all of these could be lumped into one velocity measurement where an inlet velocity spectrum and mean velocity could completely describe the flow conditions, thereby providing input to the controller for a target tip speed ratio. Currently, this would not be possible, as sites are generally evaluated for their mean velocities only, mainly due to the low sampling rates (on the order of $1 \mathrm{~Hz}$ or less) of commonly used Acoustic Doppler Current Meters or Profilers (ACMs or ADCPs). For example, in the Piscataqua River/Great Bay Estuary the last (long-term) tidal current survey was conducted with one ADCP measurement every 6 minutes (Kammere, 2007).

A stakeholder meeting at UNH-CORE with ocean renewable energy technology developers revealed that there seems to be a significant demand to get these devices out into the field for testing. However, doing so before getting plenty of data from controlled scaled laboratory experiments increases risk of failure. Deploying a device in open water is a larger investment and should occur after its performance has been optimized in a laboratory setting.

\subsection{Objectives}

First, it was of interest to measure and compare steady state, steady flow performance of a Lucid GHT and Lucid Spherical Turbine (LST), of similar size and construction. To do this, a test bed for these types of turbines was developed using the UNH Tow \& Wave Tank. Next, the effects of progressive waves on GHT performance were observed and an 
attempt was made to explain the results. Next, it was of interest to observe the effects of isotropic homogeneous turbulence on the GHT. Lastly, the effects on GHT performance from a cylinder wake inflow with diameter on the order of turbine blade chord were observed. 


\section{Cross-Flow Axis Hydrokinetic Turbines: A Theoretical Overview}

\subsection{Capturing Fluid Kinetic Energy - Single Actuator Disk Theory}

To begin analyzing a turbine in a free flow, the "highest level" analytical tool is onedimensional momentum, or "actuator disk" theory, originally developed for analyzing propeller performance by Rankine in 1865 and Froude in 1887 (Carlton, 2007). Like a propeller or in-stream axis wind turbine, a cross-flow axis hydrokinetic turbine can be approximated as an infinitesimally thin actuator disk removing a fluid's momentum kinetic energy inside a control volume enclosed within a "streamtube," shown in Figure 2.1 (Manwell, McGowan, \& Rogers, 2002). The streamtube is, in a way, a generalization of the concept of a streamline; Velocities and fluid properties are assumed to be constant over the entire cross-section at any given streamwise location, and no fluid crosses the boundary of the streamtube.

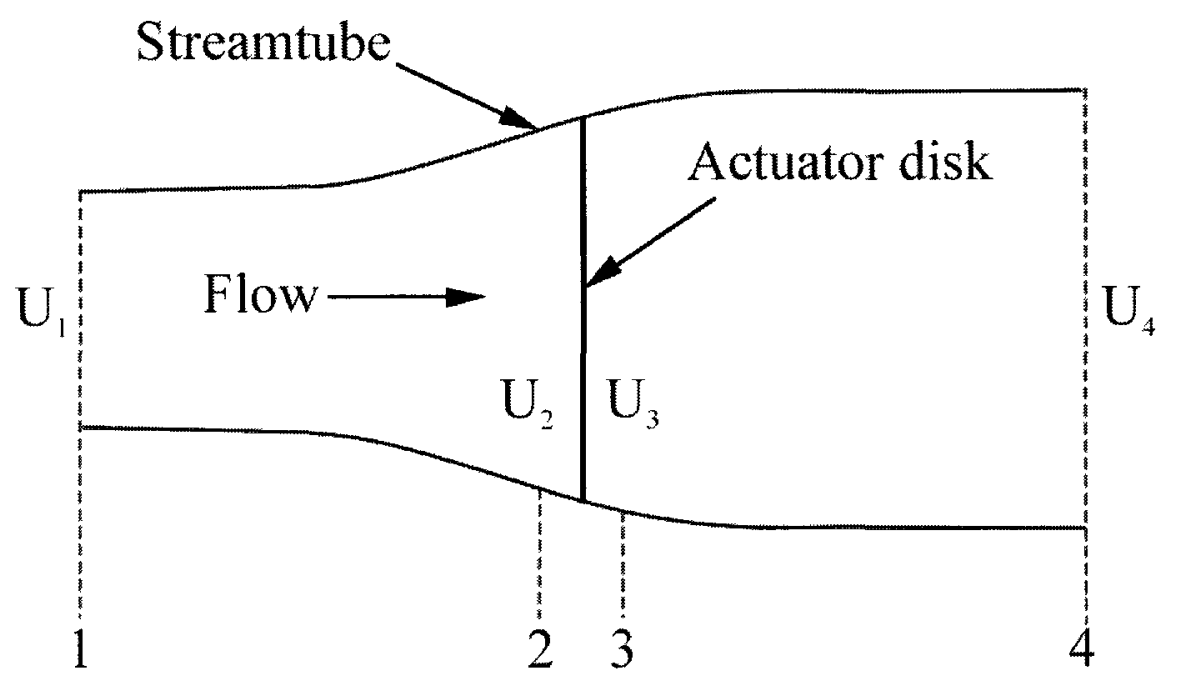

Figure 2.1 Representative control volume. Adapted from Manwell, McGowan, \& Rogers (2002). 
Control volume analysis in general can be performed using the Reynolds transport theorem:

$$
\frac{D N}{D t}=\frac{d}{d t} \int_{V} \rho \eta d V+\int_{S} \rho \eta(\vec{U} \cdot \vec{n}) d A,
$$

where $\eta$ is any quantity $N$ per unit mass and $\vec{n}$ is a unit vector normal to a control surface (Fox, Pritchard, \& McDonald, 2009). If the quantity $N$ is chosen to be the mass inside the control volume, conservation of mass for the incompressible, steady, uniform, onedimensional flow becomes

$$
A_{1} U_{1}=A_{2} U_{2}=A_{4} U_{4} \text {, }
$$

where $U_{1}$ is the free stream velocity and $A$ is the cross sectional area of the streamtube, $A_{2}$ being the turbine frontal area. The velocities just before and just after the disk, $U_{2}$ and $U_{3}$, must be equal to satisfy continuity since the disk is assumed to be infinitesimally thin. To simplify analysis, the concept of a streamwise induction factor is introduced. The streamwise induction factor, $a$, is defined as the fractional decrease in streamwise velocity from the free stream to the rotor:

$$
a=\frac{U_{1}-U_{2}}{U_{1}} .
$$

Thus fluid velocity at the disk is

$$
U_{2}=(1-a) U_{1} \text {. }
$$

Using the Reynolds transport theorem for the rate of change of fluid momentum (in other words, $N=m U$ ), the drag force on the control volume, equal to the thrust force on the disk, is simply 


$$
F_{\text {drag }}=\rho A_{1} U_{1}^{2}-\rho A_{4} U_{4}^{2} .
$$

Equation (2.2) allows this to be rearranged as

$$
F_{\text {drag }}=\rho A_{2} U_{2}\left(U_{1}-U_{4}\right)
$$

The drag force is also equal to the disk area times the pressure difference from location 2 to 3. These pressures can be solved for using Bernoulli's equation. Bernoulli's equation is derived by integrating the Euler equations (Navier-Stokes equations without viscous terms) along a streamline. It is an integration of a conservation equation for the rate of change of momentum (i.e. a force), and therefore it becomes an equation governing the conservation of mechanical energy for an inviscid flow. Since a streamtube is a spatial generalization of a streamline, Bernoulli's equation can be written between any two cross-stream planes of the streamtube, excluding the rotor. Using the Bernoulli equation to equate mechanical energy at locations on either side of the disk produces

$$
p_{1}+\frac{1}{2} \rho U_{1}^{2}=p_{2}+\frac{1}{2} \rho U_{2}^{2}
$$

and

$$
p_{3}+\frac{1}{2} \rho U_{3}^{2}=p_{4}+\frac{1}{2} \rho U_{4}^{2} .
$$

Assuming that the static pressure in the wake $\left(p_{4}\right)$ has returned to that in the free stream, the drag force becomes

$$
F_{\text {drag }}=\frac{1}{2} \rho A_{2}\left(U_{1}^{2}-U_{4}^{2}\right)
$$

The drag force can also be defined using a non-dimensional drag coefficient as:

$$
F_{\text {drag }}=\frac{1}{2} \rho C_{d} A_{2} U_{1}^{2}
$$


Solving for the drag coefficient using Equation (2.9) and Equation (2.10) yields

$$
C_{d}=1-\frac{U_{4}^{2}}{U_{1}^{2}} .
$$

The wake velocity $U_{4}$ is solved as

$$
U_{4}=(1-2 a) U_{1}
$$

which when substituted back into Equation (2.11) allows the drag coefficient to be expressed in terms of the streamwise induction factor as

$$
C_{d}=4 a(1-a)
$$

The power removed by the actuator disk is equal to the difference in kinetic energy flux between $A_{1}$ and $A_{4}$, or the drag force times the fluid velocity at the disk, $U_{2}$, written as

$$
P_{d ı k}=\frac{1}{2} \rho A_{2} U_{2}\left(U_{1}^{2}-U_{4}^{2}\right)
$$

Similar to the definition for the drag coefficient, shaft power is also defined using a nondimensional power coefficient as

$$
P_{\text {,haft }}=\frac{1}{2} \rho C_{p} A_{2} U_{1}^{3},
$$

which in this model is equal to the disk power. Power coefficient can then be expressed in terms of the streamwise induction factor only, as

$$
C_{p}=\frac{U_{2}\left(U_{1}^{2}-U_{4}^{2}\right)}{U_{1}^{3}}=4 a(1-a)^{2}
$$

Differentiating the power coefficient relation with respect to $a$ and finding where $d C_{p} / d a=0$ is how the traditional maximum power coefficient, or Betz limit, 16/27 $(59 \%)$ is calculated (Manwell, McGowan, \& Rogers, 2002). 
While the power coefficient is the fraction of total kinetic power in the flow converted to shaft power, in this simple model there are no losses. Therefore the kinetic power flux at the outlet is that at the inlet minus the power out at the actuator disk. Of course, real world operation will not be ideal, and for the simple one-dimensional momentum theory any induction factor above 0.5 will theoretically produce a negative wake velocity. Despite its shortcomings, one-dimensional momentum theory can be used to define and estimate a "second law efficiency," or the fraction of the (linear) kinetic energy removed from the flow (the available energy, or "exergy") that is actually converted to shaft work.

First, the second law efficiency or kinetic exergy efficiency is defined as

$$
\eta_{I I}=\frac{P_{\text {shaft }}}{P_{d s k k}},
$$

where the rate of energy extracted by the rotor disk is calculated as drag (thrust) force time velocity at the rotor. Using Equation (2.14) and (2.15), this can be written as

$$
\eta_{I I}=\frac{C_{p} U_{1}^{3}}{U_{2}\left(U_{1}^{2}-U_{4}^{2}\right)} .
$$

In terms of drag coefficient, power coefficient, and induction factor this becomes

$$
\eta_{I I}=\frac{C_{p}}{(1-a) C_{d}} .
$$

By definition, the ideal actuator disk described by Equations (2.13) and (2.16) operates at $100 \%$ kinetic exergy efficiency. For a real device, knowing the power coefficient and kinetic exergy efficiency allows prediction of power output and kinetic energy flux out of the turbine, which is important for environmental modeling and for predicting performance when turbines are used in arrays. It is assumed that any kinetic 
power removed from the fluid that is not converted to shaft work is redistributed into heat "or turbulent kinetic energy in the wake. This energy may or may not be available for subsequent turbines to convert into shaft work.

\subsection{Blockage Effects and Correction}

Putting a turbine in a constrained flow such as a channel or pipe changes the assumption used to derive Betz theory, not allowing streamlines to diverge as freely, therefore increasing velocity at the turbine. A correction can be derived from the blocked actuator disc model shown in Figure 2.2 by using the principles of continuity and conservation of momentum. The following derivation is taken from Appendix A of Bahaj, Molland, Chaplin, \& Batten (2007). Note that this correction does not take into account the deformation of the free surface at the top of the channel or tank. 


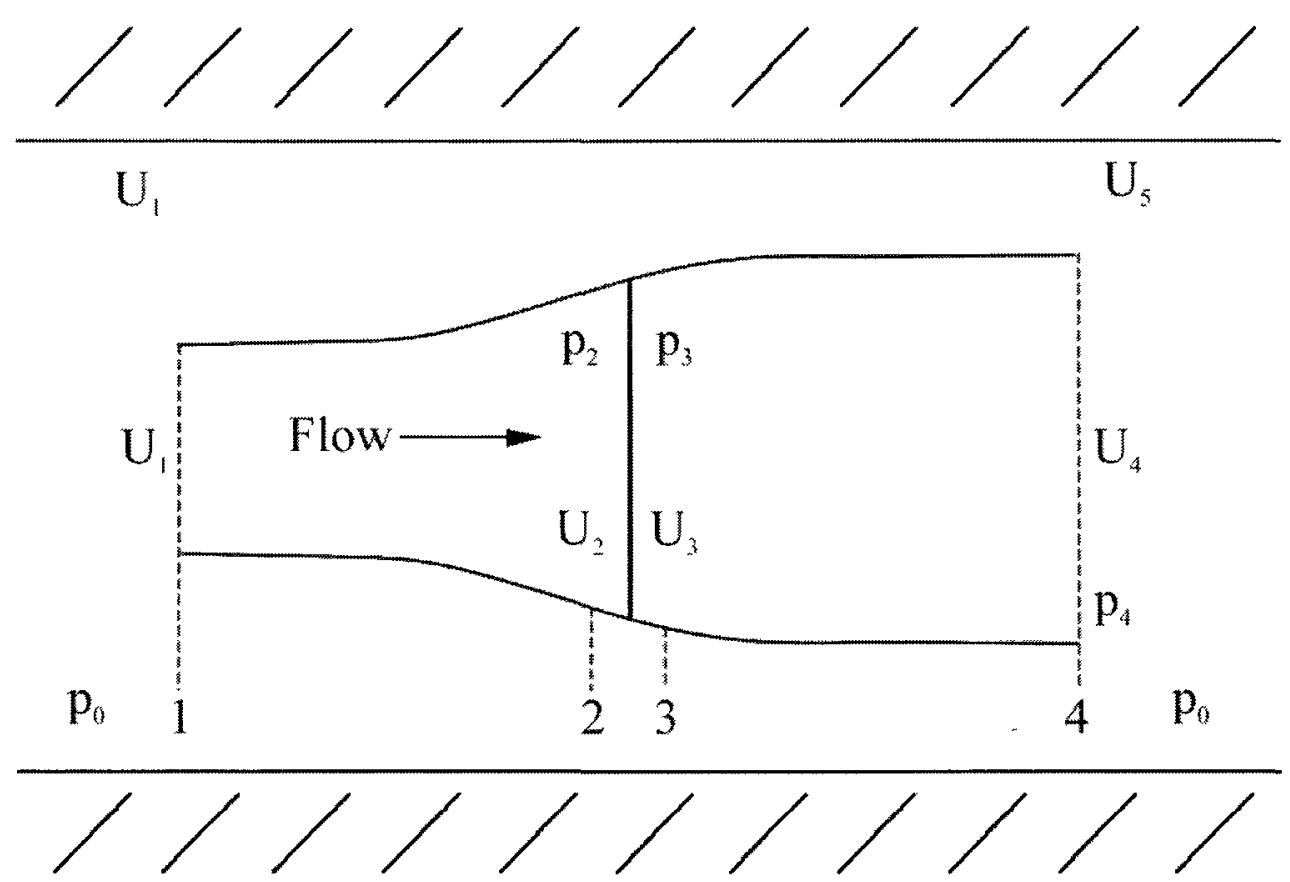

Figure 2.2 Turbine control volume in a blocked flow. Adapted from Bahaj, Molland, Chaplin, \& Batten, (2007).

First, from continuity the ratio of disk velocity to wake velocity is written as

$$
\frac{U_{2}}{U_{4}}=\frac{-1+\sqrt{1+\left(A_{2} / A_{c}\right)\left(\left(U_{5} / U_{4}\right)^{2}-1\right)}}{\left(A_{2} / A_{c}\right)\left(\left(U_{5} / U_{4}\right)-1\right)}
$$

where $A_{c}$ is the tank cross-sectional area. Next, the ratio of free stream velocity to wake velocity can be written as

$$
\frac{U_{1}}{U_{4}}=\frac{U_{5}}{U_{4}}-\frac{A_{2}}{A_{c}} \frac{U_{2}}{U_{4}}\left(\frac{U_{5}}{U_{4}}-1\right) .
$$

Using conservation of momentum, the drag coefficient in the tank or channel becomes

$$
C_{d}=\left(\frac{U_{4}}{U_{1}}\right)^{2}\left(\left(\frac{U_{5}}{U_{4}}\right)^{2}-1\right)
$$

Therefore, the ratio of free stream to wake velocity is 


$$
\frac{U_{1}}{U_{4}}=1 / \sqrt{\frac{C_{d}}{\left(\left(U_{5} / U_{4}\right)^{2}-1\right)}} .
$$

The main principle of correction is that blocked turbine performance will be equivalent when $U_{2}$, turbine RPM, and drag force are the same as they would be in free flow. By iteratively solving Equation (2.21) and Equation (2.23) to find the ratio $U_{5} / U_{4}$, then substituting this ratio back into Equation (2.20), the ratio $U_{2} / U_{1}$ can be found. This ratio is then used to calculate the ratio of tank velocity to an equivalent free flow velocity

$$
\frac{U_{1}}{U_{f}}=\frac{U_{2} / U_{1}}{\left(U_{2} / U_{1}\right)^{2}+C_{d} / 4}
$$

Using this velocity ratio, turbine tip speed ratio, drag coefficient, and power coefficient can be corrected as

$$
\begin{gathered}
\left.\lambda\right|_{\text {free }}=\left.\left(\frac{U_{1}}{U_{f}}\right) \lambda\right|_{\text {blocked }}, \\
\left.C_{d}\right|_{\text {free }}=\left.\left(\frac{U_{1}}{U_{f}}\right)^{2} C_{d}\right|_{\text {blocked }},
\end{gathered}
$$

and

$$
\left.C_{p}\right|_{\text {free }}=\left.\left(\frac{U_{1}}{U_{f}}\right)^{3} C_{p}\right|_{\text {blocked }} .
$$

Note that this type of correction, namely one with a constant induction across the actuator disk, may overcorrect for CFA turbines, due to their inherently non-uniform induction characteristics (Ferreira, van Kuik, van Bussel, \& Scarano, 2009). 


\subsection{Flow and Forces on a Turbine Blade}

The fluid dynamics of the lift driven CFA turbines of interest to this study are approximated by a number of $2 \mathrm{D}$ foils moving in a circular path with a superimposed uniform flow. Since foil lift and drag coefficient data are available for many common shapes, this is a useful first estimate at predicting blade forces, and from those torque and power. By vector addition of the negative of the blade tangential velocity to the induced (or slowed) free stream, the turbine blade angle of attack can be calculated. Since the blade tangential velocity is constantly changing direction throughout its path, the turbine blade angle of attack and incident fluid velocity, or relative velocity, are likewise oscillating throughout the turbine's rotation.

Turbine operation can be prescribed by one nondimensional operating parameter, the tip speed ratio, $\lambda$. It is defined as the ratio of blade tangential velocity to free stream velocity. It can be thought of as non-dimensional rate of rotation, written as

$$
\lambda=\frac{\omega r}{U_{\infty}},
$$

where $\omega$ is the turbine shaft angular velocity, $r$ is the radius of the turbine, and $U_{\infty}$ is the free stream velocity. Two examples of velocity vector and force diagrams are shown in Figure 2.3, one (top) for an unstalled foil at an azimuthal angle $\theta=45$ degrees, and one (bottom) for a stalled foil at $\theta=100$ degrees. The diagrams were drawn for an approximate tip speed ratio of 2 , with a streamwise induction factor of $1 / 3$. 

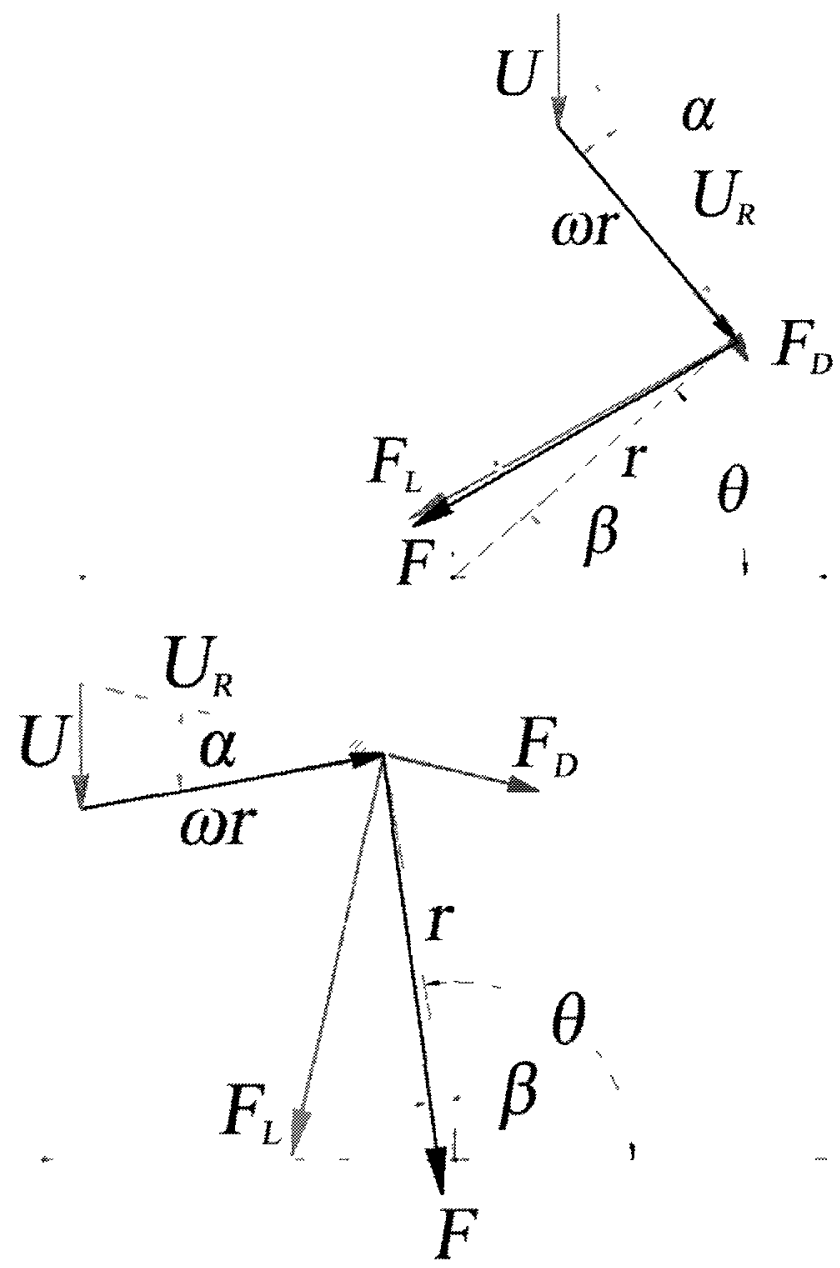

Figure 2.3 Blade geometry and force diagrams for unstalled (top) and stalled (bottom) conditions. $\theta, \alpha$, and $\beta$ are turbine blade azimuthal angle, angle of attack, and angle between resultant force $F$ (the vector addition of lift force $F_{L}$ and drag force $F_{D}$ ) and the radius $r$, respectively. $U$ is the free stream velocity multiplied by $(1-a)$, where $a$ is the streamwise induction factor. $\omega r$ is the negative of the blade tangential velocity. $U_{R}$ is the relative velocity, calculated by the vector addition of $\omega r$ and $U$.

Using the geometry shown in Figure 2.3, turbine blade angle of attack can be calculated as

$$
\alpha=\tan ^{-1}\left(\frac{U \sin \theta}{\omega r+U \cos \theta}\right)
$$


where $U$ is the free stream velocity multiplied by $(1-a)$, where $a$ is the previously defined streamwise induction factor. Relative fluid velocity seen by the blade is the vector subtraction of the blade velocity from the induced free stream,

$$
\vec{U}_{R}=\vec{U}-\omega \vec{r},
$$

which has the magnitude

$$
\left|\vec{U}_{R}\right|=\sqrt{U^{2}+2 U \omega r \cos \left(\theta+(\omega r)^{2}\right)}
$$

Fluid dynamic lift and drag are defined as

$$
F_{L} \equiv \frac{1}{2} \rho A_{p} C_{l} U_{R}^{2}
$$

and

$$
F_{D} \equiv \frac{1}{2} \rho A_{p} C_{d} U_{R}^{2}
$$

respectively, where $A_{p}$ is the planform area of the blade, equal to the chord length times the blade span. The angle between the radius and resultant fluid force can be expressed as

$$
\beta=\alpha-\tan ^{-1}\left(\frac{F_{D}}{F_{L}}\right)
$$

making the torque about the turbine axis

$$
T=\operatorname{Fr} \sin (\beta)
$$

When the angle $\beta$ is larger, a larger component of the hydrodynamic force acts to produce torque about the axis of rotation. This means that to produce maximum torque, angle of attack and lift to drag ratio should be as high as possible. However, these two criteria conflict with each other to the right of the peaks of the curves shown in Figure 2.6, especially after the stall point, or angle at with lift to drag ratio decreases sharply. 
Looking again at the diagram in Figure 2.3, the larger drag force associated with a stalled blade reduces the angle, $\beta$, between the radius and resultant force, and can even turn it negative, if drag is high enough. Following this logic, foils with high stall angles are ideal for CFA turbines, such that maximum angle of attack throughout a rotation can remain high without stall, keeping $\beta$ as high as possible.

Figure 2.4 and Figure 2.5 show turbine blade angle of attack and relative velocity, respectively, plotted versus turbine azimuthal angle for one blade throughout one rotation using the linear induction relationship estimate $a=0.1838 \lambda+0.1623$, the justification for which is discussed in Chapter 5. Using this induction estimate, the amplitude of the angle of attack and relative velocity oscillation decreases with tip speed ratio more quickly than it would with a constant induction factor. Nevertheless, the unsteady flow behavior around a blade of a CFA turbine is apparent. Note that this calculation doesn't take into account the slower velocity seen by the downstream half of the blade's rotation, due to the fluid interaction with the first half of the rotation. This will effectively decrease the angle of attack and relative velocity on the downstream half of the rotor. 


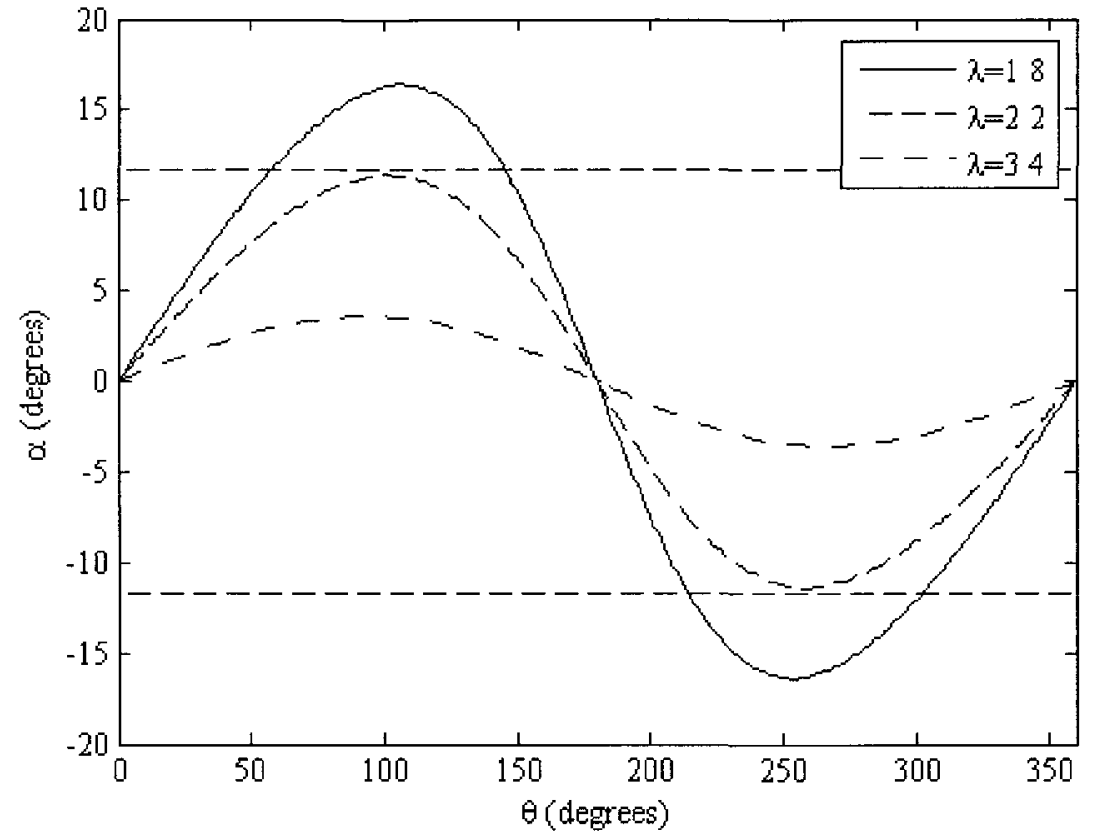

Figure 2.4 Theoretical turbine blade angle of attack at various tip speed ratios, including estimated induction. Dashed horizontal lines show typical static stall angle.

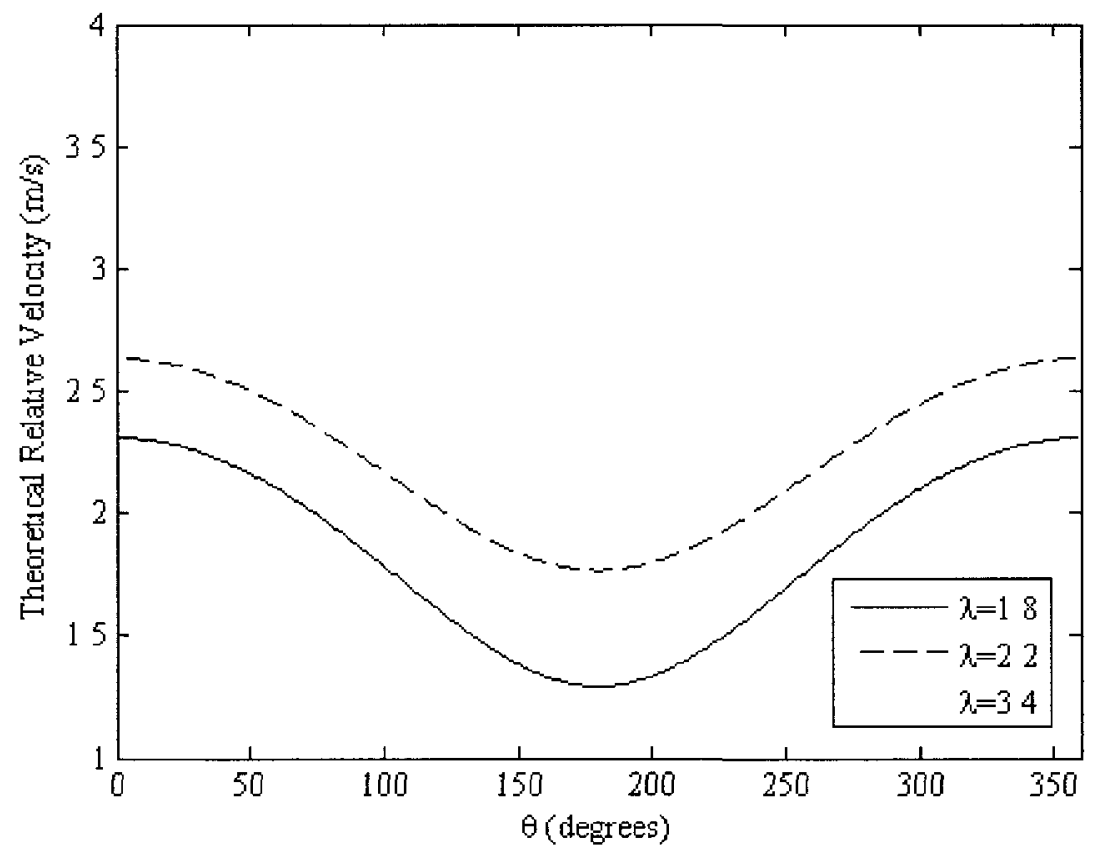

Figure 2.5 Plot of theoretical relative velocity seen by a turbine blade throughout one rotation in a free stream velocity of $1 \mathrm{~m} / \mathrm{s}$. 
Figure 2.6 shows various NACA symmetrical foils' lift to drag ratio versus angle of attack for a chord Reynolds number of 700,000 , taken from a report by Sandia National Laboratories (Sheldahl \& Klimas, 1981). The last two digits of the foil number are the thickness of the foil as a percentage of the chord length. For each foil, static stall angles of attack can be identified as the angle at which the lift to drag ratio sharply decreases with increasing angle of attack. In general, for these foils a thinner profile maximizes lift to drag ratio at the expense of a lower static stall angle.

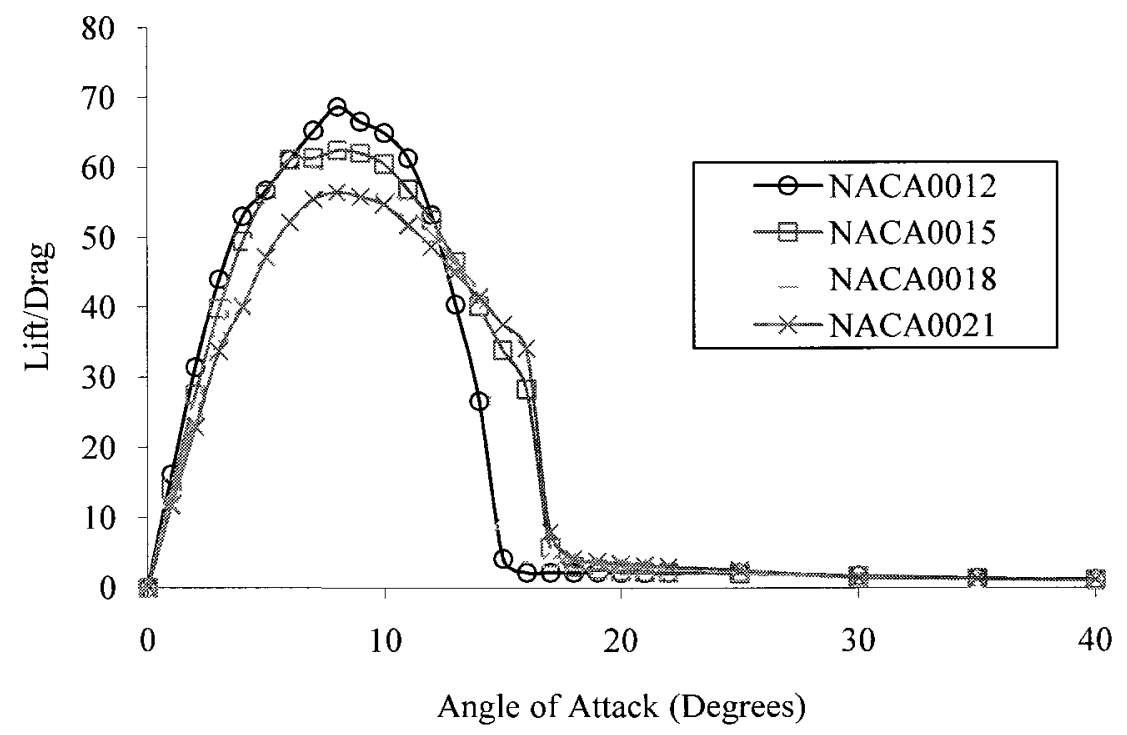

Figure 2.6 Lift to drag ratio versus angle of attack for various airfoils at $\mathrm{Re}_{\mathrm{c}}=$ 700,000 (Sheldahl \& Klimas, 1981).

So far, consideration has only been given to static stall criteria. Though this is a good place to start, an oscillating foil undergoes dynamic stall in certain conditions. Dynamic stall is an unsteady phenomenon occurring when a foil operates with an angle of attack oscillation whose amplitude exceeds the foil's static stall angle. Examining its effects on a turbine blade is further complicated by the periodic unsteadiness of the relative velocity seen by the blade. However, in general the phenomenon is characterized 
by a separated flow causing an initial increase or "overshoot" in lift and drag beyond static values, followed by an "undershoot" where lift and drag fall below static values before flow reattaches to the foil. Despite this "undershoot," dynamic stall generally improves performance in models (Paraschivoiu, 2002). In general, dynamic stall occurs at angles of attack beyond those of static stall, depending on mean angle, rate of oscillation, foil shape, and Reynolds number (Sheng, Galbraith, \& Coton, 2008). Datasets for foils in dynamic stall experiments are difficult to apply to CFA turbines since they are usually performed with a foil in a sinusoidal angle of attack oscillation in a constant free stream, whereas a CFA turbine blade sees an oscillation that is not quite sinusoidal, along with an oscillation in relative velocity.

There have been studies to visualize and measure the velocity field around a foil in Darrieus motion during dynamic stall (Ferreira, van Kuik, van Bussel, \& Scarano, 2009) (Fujisawa \& Shibuya, 2001). In general, dynamic stall is characterized by the shedding of two counter-rotating vortices that are convected downstream, which may or may not interact with blades on the downstream half of the rotor, depending on tip speed ratio.

\subsection{Foil Performance in Turbulence}

Since any real world application of these turbines will no doubt be in turbulent flow, it is important for turbine performance prediction to understand how individual foils perform in similar turbulent flows. In general, turbulence can delay separation on the foil's suction side, and hence stall, but will also increase drag on the foil due to increased momentum transport by the turbulence. 
Experiments were performed measuring lift and drag on a NACA0021 foil in various levels of grid turbulence. A delay of static stall to higher angles of attack was observed with increasing turbulence intensity (Swalwell, Sheridan, \& Melbourne, 2001).

As for the effects of grid turbulence on dynamic stall, the lift coefficient "undershoot" is reduced (Amandolese \& Szechenyi, 2004). It follows that grid turbulence would have a positive effect on CFA performance.

Larger scale turbulence or vortices on the same scale as the vorticity created by a blade in dynamic stall would have a drastic effect, which could only be predicted if the convection velocity and phase of the vortices were known. Oscillating foils have been shown to be able to achieve high propulsive efficiency (Anderson, Streitlien, Barrett, \& Triantafyllou, 1998). Also, foils have been shown to produce thrust without any energy input when placed in the wake of a bluff body (Beal, 2006). However, this situation is somewhat different from what will happen when a CFA turbine is downstream of a bluff body, where an individual blade will not be directly downstream of the object's wake at all times.

\subsection{Rotating Rigid Body Dynamics}

The dynamics of the entire turbine assembly are approximated by a single hydrodynamic torque resisted by a load torque (including bearing friction or tare torque), the difference between which being equal to the rate of change of angular momentum of the device, written as

$$
J \dot{\omega}=T_{\text {flud }}-T_{\text {load }},
$$


where $J$ is the rotational inertia of the device about its axis. This shows that in order to measure hydrodynamic performance, it is necessary to measure steady state performance to avoid the additional uncertainty of estimating the rotational inertia of the system. In other words, measurements should be taken when the angular velocity of the rotor is approximately constant.

\subsection{Cross-Flow Axis Turbine Design Parameters}

One important design parameter for a cross-flow axis hydrokinetic turbine is its solidity, defined as the ratio of total blade planform area to swept area, which can be written as

$$
\sigma=\frac{N c L_{s}}{A_{s}},
$$

where $N$ is the number of blades, $c$ is the chord length perpendicular to the blade span, $L_{s}$ is the blade span length, and $A_{s}$ is the turbine swept area. Note that other definitions for solidity for Darrieus devices occur in the literature. For example, Paraschivoiu defines solidity as the ratio of total chord length to radius (Paraschivoiu, 2002). This definition is less useful for non-cylindrical devices since their radii vary with height. For this reason, the area ratio definition of Equation (2.37) was used in this study.

Another parameter for helical devices is blade overlap. This is the ratio of how much total blade span is projected onto the circumference of the device's rotation, which is related to helical sweep angle and turbine height.

\subsection{Linear Progressive Surface Wave Theory}

A progressive surface wave induces an elliptical motion in the fluid particles below whose amplitude decreases with depth from the equilibrium surface. When a turbine is 
operated beneath a wave, this wave induced velocity is superimposed upon the free stream. The elliptical fluid trajectories beneath a progressive wave can be derived from linear (Airy) wave theory using a velocity potential formulation. The horizontal component of this velocity is then found to be

$$
u=\frac{g H k}{2 \sigma} \frac{\cosh k(h+z)}{\cosh k h} \cos (k x-\sigma t),
$$

where $g$ is the gravitational acceleration, $H$ is the wave height from crest to trough, $\sigma$ is the radian frequency of the wave, $k$ is the wavenumber, $h$ is depth, $z$ is depth from equilibrium surface, $x$ is the spatial coordinate in the direction of propagation, and $t$ is time (Dean \& Dalrymple, 1991).

For this study, the turbine will be mounted such that its axis of rotation is oriented vertically in the plane of wave-induced fluid particle motion. Other wave energy conversion research including working scale models has been performed using a Darrieus turbine with its axis oriented horizontally while perpendicular to the plane of wave induced fluid particle motion, for example (Siegel, Jeans, \& McLaughlin, 2010). Whales have also been shown to use their flukes to capture some of the energy in waves for their own propulsion (Bose \& Lien, 1990). 


\section{Development of a Hydrokinetic Turbine Test Bed for the UNH Tow \& Wave Tank}

Laboratory testing in a large cross-section tow tank or flume presents an opportunity to test turbines in a more controlled, less expensive way compared to open-water deployment, for example at the UNH-CORE Tidal Energy Test Site. In Fall 2009, the idea for using the UNH Tow and Wave Tank for testing hydrokinetic turbines was conceived.

\subsection{Turbines Tested}

Lucid Energy Technologies, LLP sent their Gorlov Helical Turbine (GHT) and Lucid Spherical Turbine (LST) to the UNH Center for Ocean Renewable Energy for performance evaluation under an industry research project, and the test bed was designed around their dimensions. The two turbines are shown in Figure 3.1 and turbine specifications are presented in Table 3.1. Both turbines are made from extruded aluminum NACA 0020 foil sections with $14 \mathrm{~cm}$ chord length. However, the blades of the GHT are technically not a NACA 0020 swept along a helix but the foil tilted at the helix angle and wrapped around cylindrically. Therefore, the $2 \mathrm{D}$ blade section in the plane of rotation is not a NACA 0020 . The actual projected foil shape of the GHT is shown in Figure 3.2. The GHT has an effective chord along its blade path that is higher due to the method of construction. Taking the tilt into consideration, the thickness is roughly $19 \%$ of the projected $15 \mathrm{~cm}$ chord, making the foil more like a NACA 0019 in its plane of motion. Similarly, the LST is not technically a helical sweep, but a foil bent in a circular 
arc then tilted at the helix angle. The LST foil projections are more complicated, varying with height, depicted at the equator, quarter-height, and top/bottom locations in Figure 3.3, Figure 3.4, and Figure 3.5, respectively. Technically, along its path, the projected blade sections closer to the top and bottom of the LST look like cambered sections, with progressively larger camber and chord length further from the equator. Since the LST's radius is not constant, local tip speed ratio and local (two-dimensional) solidity vary with height, with the highest tip speed ratio and lowest solidity located at the equator. This also illustrates the difficulty of estimating performance simply from the NACA foil data given in (Sheldahl \& Klimas, 1981). Note that the solidity of the LST was calculated using the CAD models provided by Lucid to measure blade span and swept area, since the LST is not perfectly spherical.
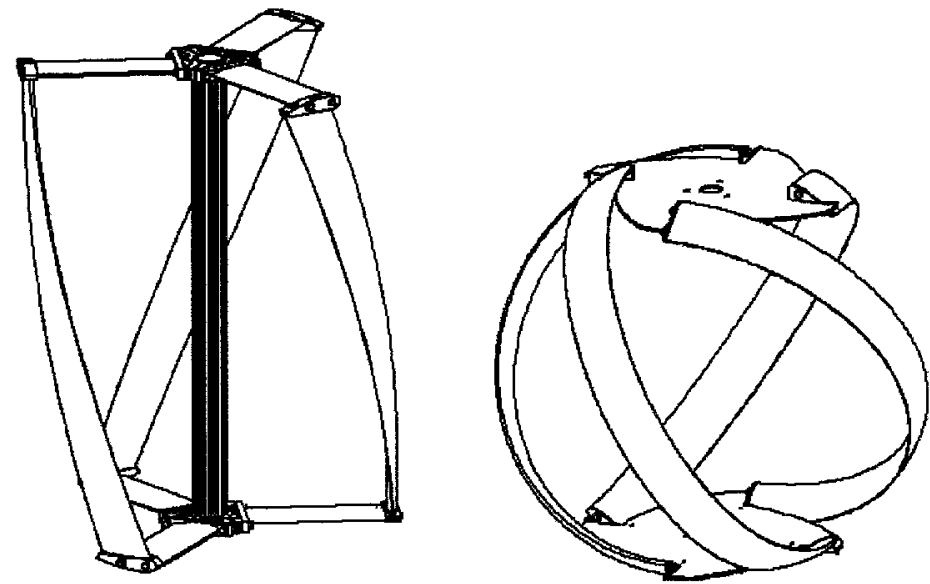

Figure 3.1 Sketch of the GHT (left) and LST (right). 
Table 3.1 Turbine specifications.

\begin{tabular}{lll}
\hline & GHT & LST \\
\hline Diameter $(\mathrm{m})$ & 1.00 & 1.14 \\
Height $(\mathrm{m})$ & 1.32 & 0.97 \\
Blade profile & NACA 0020 & NACA 0020 \\
Chord $(\mathrm{cm})$ & 14 & 14 \\
Solidity & 0.14 & 0.22 \\
$A_{f}\left(\mathrm{~m}^{2}\right)$ & 1.32 & 0.96 \\
Blade overlap & $1 / 2$ & 2 \\
\hline
\end{tabular}

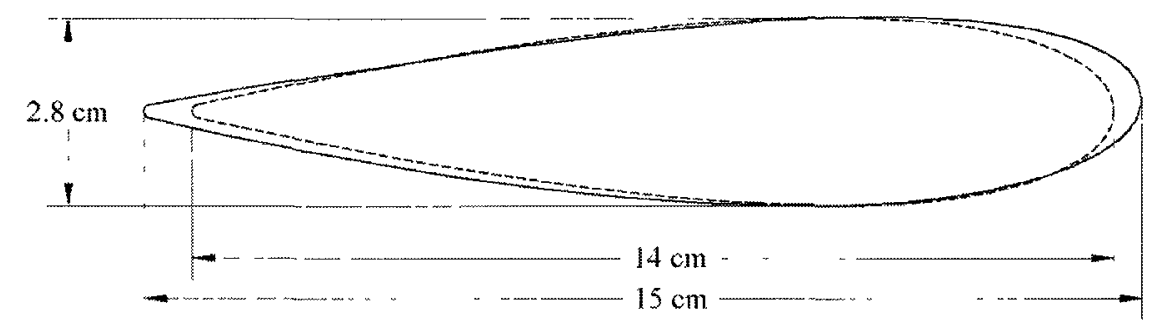

Figure 3.2 Projected GHT foil shape along counter-clockwise path. Radius points upwards. Dashed lines are non-projected NACA 0020.

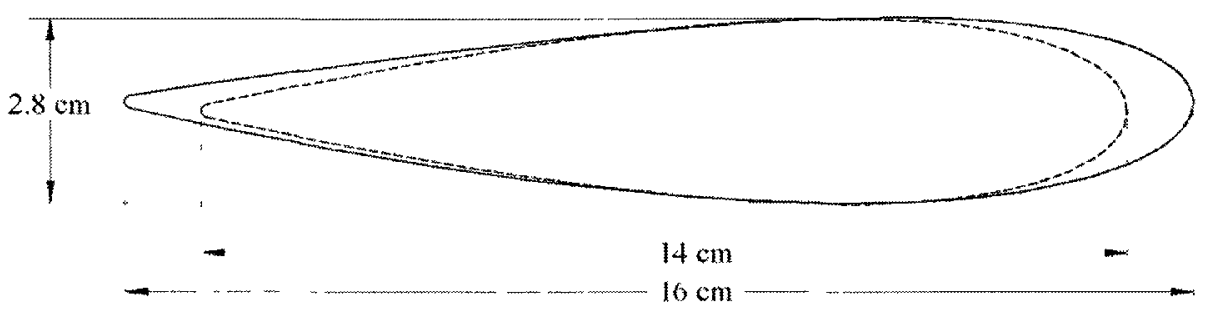

Figure 3.3 Projected LST foil shape along counter-clockwise path at equator. Radius points upwards. Dashed lines are non-projected NACA 0020. 


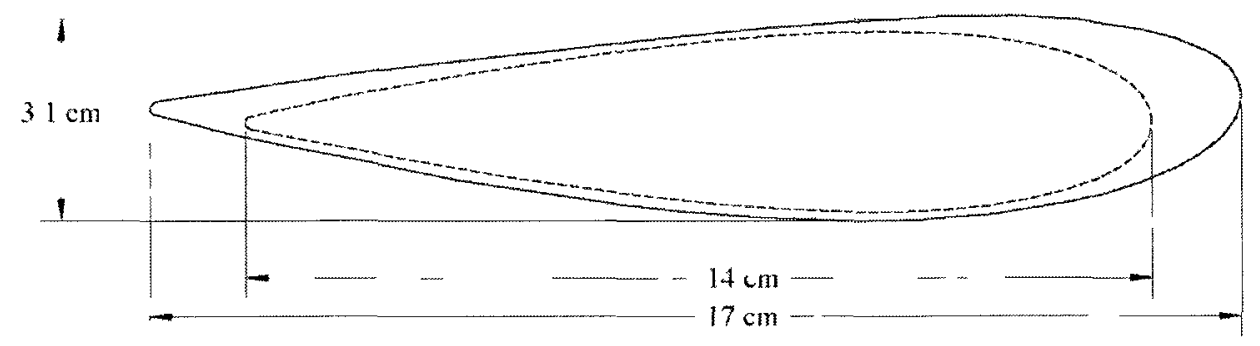

Figure 3.4 Projected LST foil shape along counter-clockwise path at $1 / 4$ height. Radius points upwards. Dashed lines are non-projected NACA 0020.

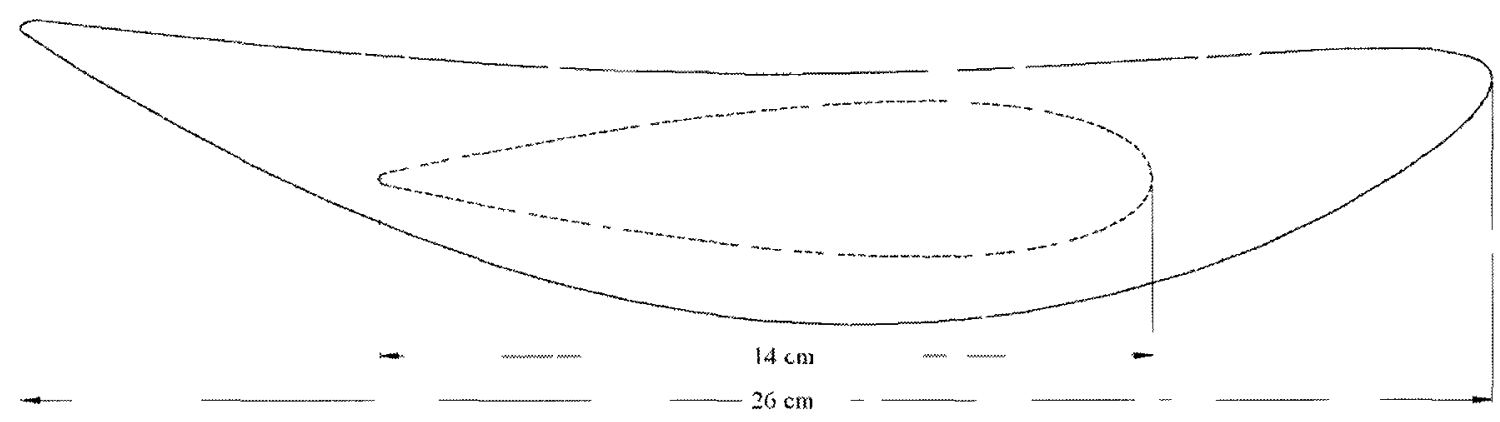

Figure 3.5 Projected LST foil shape along counter-clockwise path near the poles. Radius points upwards. Dashed lines are non-projected NACA 0020.

\subsection{The UNH Tow \& Wave Tank}

The UNH Tow \& Wave Tank, shown in Figure 3.6, located at the Chase Ocean Engineering Laboratory, was constructed in 1994. The carriage and tow mechanism were designed and built as part of a master's thesis, completed in 1996 (Darnell, 1996). It is a

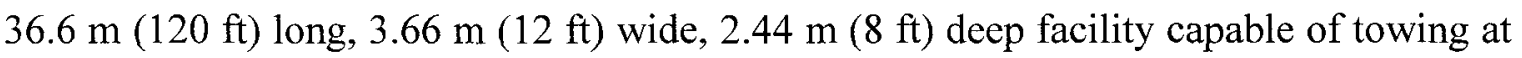
speeds up to $7 \mathrm{~m} / \mathrm{s}$ and a flap style wave maker at one end is capable of producing waves with $1-5 \mathrm{~s}$ periods up to $0.4 \mathrm{~m}$ wave height.

The tow mechanism consists of a Yaskawa V7 drive powering a Baldor $10 \mathrm{hp} \mathrm{AC}$ motor run through a Dodge Maxum gearbox, driving a 1/4" diameter steel cable (wire 
rope). The specifications of the system are listed in Table 3.2. The system is an open loop design, consisting of a LabVIEW VI that outputs a DC voltage to the motor drive proportional to desired speed. The drive then sends power to the motor at the correct frequency. There is no compensation for slip in the system, but velocities are fairly accurate at low to moderate loads (Darnell, 1996).

Table 3.2 Tow system specifications (Darnell, 1996).

\begin{tabular}{ll} 
Maximum speeds & $1.54,3.70$, and $7.12 \mathrm{~m} / \mathrm{s}$ (measured) \\
Max. tow force & $700 \mathrm{lbf}($ design), $507 \mathrm{lbf}$ (measured) \\
Cable preload & $1000 \mathrm{lbf}$ \\
Power limit $(\mathrm{kW})$ & $4 \mathrm{~kW}$ if $U<1.8 \mathrm{~m} / \mathrm{s}, 7.5 \mathrm{~kW}$ if $U>1.8 \mathrm{~m} / \mathrm{s}$ \\
Acceleration & $90 \%$ of max speed reached in 1,2, and $5 \mathrm{~m}$, for low, \\
& mid, and high speed ranges, respectively (measured). \\
Max. pitch torque & $3300 \mathrm{Nm}$ \\
\hline
\end{tabular}

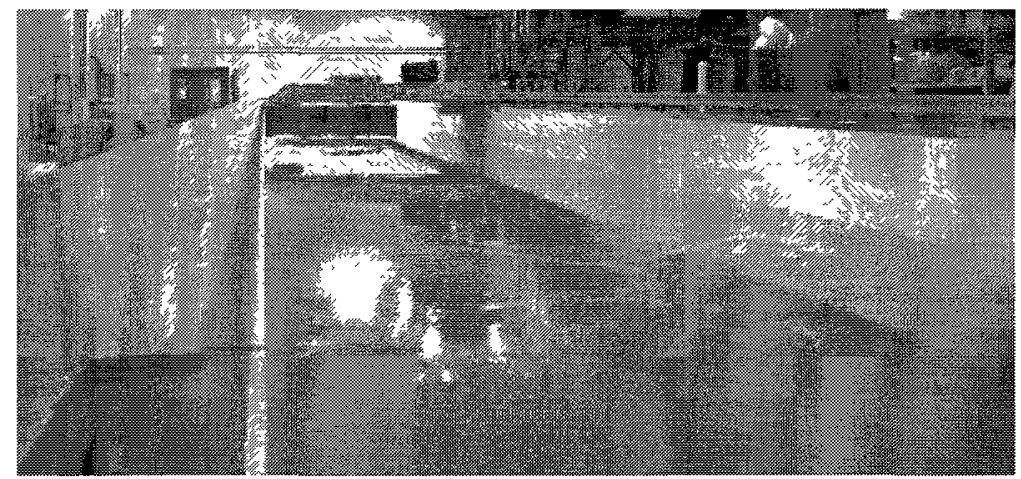

Figure 3.6 The UNH tow \& wave tank.

\subsection{Test Bed Design: Desired Characteristics and Concept Selection}

At the beginning of the project, the following desired characteristics and target metrics were established:

- Ability to tow turbines up to $1.3 \mathrm{~m}^{2}$ frontal area up to $1.5 \mathrm{~m} / \mathrm{s}, 3 \mathrm{~m} / \mathrm{s}$ for smaller turbines. 
- Measurement of shaft torque, RPM, and streamwise drag force.

- Corrosion resistance for weeks in the tank at a time.

- Ability to easily mount turbulence generators.

- No drilling or welding to carriage.

The first logical step in assessing the ability of the existing facility to accommodate the turbine test bed was to determine if the tow mechanism would be able to produce enough force and power to tow the turbines at the desired speeds. Overall turbine drag coefficient based on turbine frontal area was estimated from Equation (2.13) to be a maximum of unity. Accordingly, it was predicted that when towed at $1.5 \mathrm{~m} / \mathrm{s}$ in the tank, the GHT (worst case scenario due to its larger frontal area) would create approximately $1500 \mathrm{~N}(340 \mathrm{lbf})$ of drag force, which is below the $2250 \mathrm{~N}$ force limit measured by Darnell. At $1.5 \mathrm{~m} / \mathrm{s}$, a $1500 \mathrm{~N}$ drag force will require $2250 \mathrm{~W}$ to operate, which is also below the $4 \mathrm{~kW}$ maximum power output of the tow mechanism in this speed range.

Based on the maximum tow force, a maximum speed of $1.8 \mathrm{~m} / \mathrm{s}$ was estimated. Since this speed would require gear changing with questionable benefits (also with no factor of safety included), it was decided that a $1.5 \mathrm{~m} / \mathrm{s}$ would be the limit for tow speed until the tow mechanism was upgraded or a smaller turbine model was available.

In order to remove mechanical power from the rotating turbine, a resistive torque must be applied. A passive dynamometer style system was chosen for simplicity and avoiding the need for any sort of auxiliary energy storage method on board the carriage. Other options include a DC motor and closed loop controller, which would allow prescription of tip speed ratio and could be used to start the turbine. This concept was not chosen due to the limited electrical power onboard the tow carriage. 
For turbine drag measurements, two load cells were chosen, one for each side, as opposed to connecting both rails to one cross member and using one load cell. This was done to allow for uneven loading of either side without binding.

Lucid provided additional lengths of extruded aluminum NACA 0020 struts (the same extrusions that the turbine blades are made from), which were chosen to be the main submerged structural members. This facilitated the design of a low-drag tow frame.

To minimize the effects of blockage, the test bed was designed so that the turbine would be roughly centered in the tank cross section. The turbine frame was designed to tow in one direction, away from the wave maker. While the installed test bed could be towed in both directions, symmetrical foil support struts would be better in this case.

Since the turbine frame could possibly be submerged in the tank for a period of a week or so, corrosion was a small concern. All submerged parts are 6061-T6 aluminum, 304, or 316 stainless steel. Galvanic corrosion from contact of dissimilar metals was a slight concern, but it was not considered crucial to isolate these components due to the short submergence periods.

\subsection{Main Frame Assembly: Turbine Mounting, Loading, and RPM Measurement}

The hydrokinetic turbines mount inside the main frame assembly between two mounting flanges, whose bolt patterns were designed to match those on the Lucid turbines. The main frame assembly, shown in Figure 3.7, is also where the drive shaft, brake, torque transducer, and shaft speed sensor are located.

Since the design involved supporting the turbine, shaft, torque transducer, and couplers with the horizontal foil members, it was first confirmed that these NACA 0020 
members could withstand the bending loads imposed on them by the weight of these components. These calculations can be found in Appendix A.

An appropriate shaft was chosen to transmit power from the turbine to the loading mechanism. Based on a report by Gorlov stating maximum power coefficients of 0.35 at a tip speed ratio of 2 (Gorlov, Development of the Helical Reaction Hydraulic Turbine, 1998), maximum torque at $1.5 \mathrm{~m} / \mathrm{s}$ was estimated to be $130 \mathrm{Nm}$ at approximately 60 RPM. A 1.25 inch diameter 304 stainless steel shaft was chosen based on the criteria of moderate corrosion resistance, shear stress, and critical angular frequency. With a 130 $\mathrm{Nm}$ torque load along its axis of rotation, the shaft would incur a shear stress off approximately 3,000 psi, which is a safe shear stress for 304 stainless steel, having a yield stress of 35,000 psi in an annealed state (Deutschman, Michels, \& Wilson, 1975). The critical (resonant) speed of the shaft is calculated to be 11,000 RPM using the equation

$$
R P M_{\text {critcal }}=4,760,000 \frac{d}{l^{2}}
$$

where $d$ is the shaft diameter in inches and $l$ is the distance between bearings in inches (Oberg \& Jones, 1959). Since this is roughly two orders of magnitude higher than the expected maximum RPM, the shaft is well within safe limitations.

Flexible shaft couplers were chosen to prevent binding from any misalignment in the mechanism. The shaft has keyways for ease of coupling at each end. The turbine is supported by two flanged corrosion resistant ball bearing assemblies. The thrust loads on these main shaft bearings are low, on the order of $130 \mathrm{lbf}$, negating the need for an actual thrust bearing since the entire load would produce an estimated shear stress of 2500 psi if entirely loaded on one of the 0.27 inch diameter balls. The shaft is held from moving 
radially below the torque transducer by a low force capacity plain bearing made from oilimpregnated sintered bronze with a built-in rubber radial damper.

The GHT and LST both have similar bolt patterns at their upper and lower mating surfaces. However, the LST is shorter in height than the GHT. To allow the test frame to easily fit both turbines, a pair of identical shaft extensions were designed and fabricated to be attached to the LST to match the GHT height.

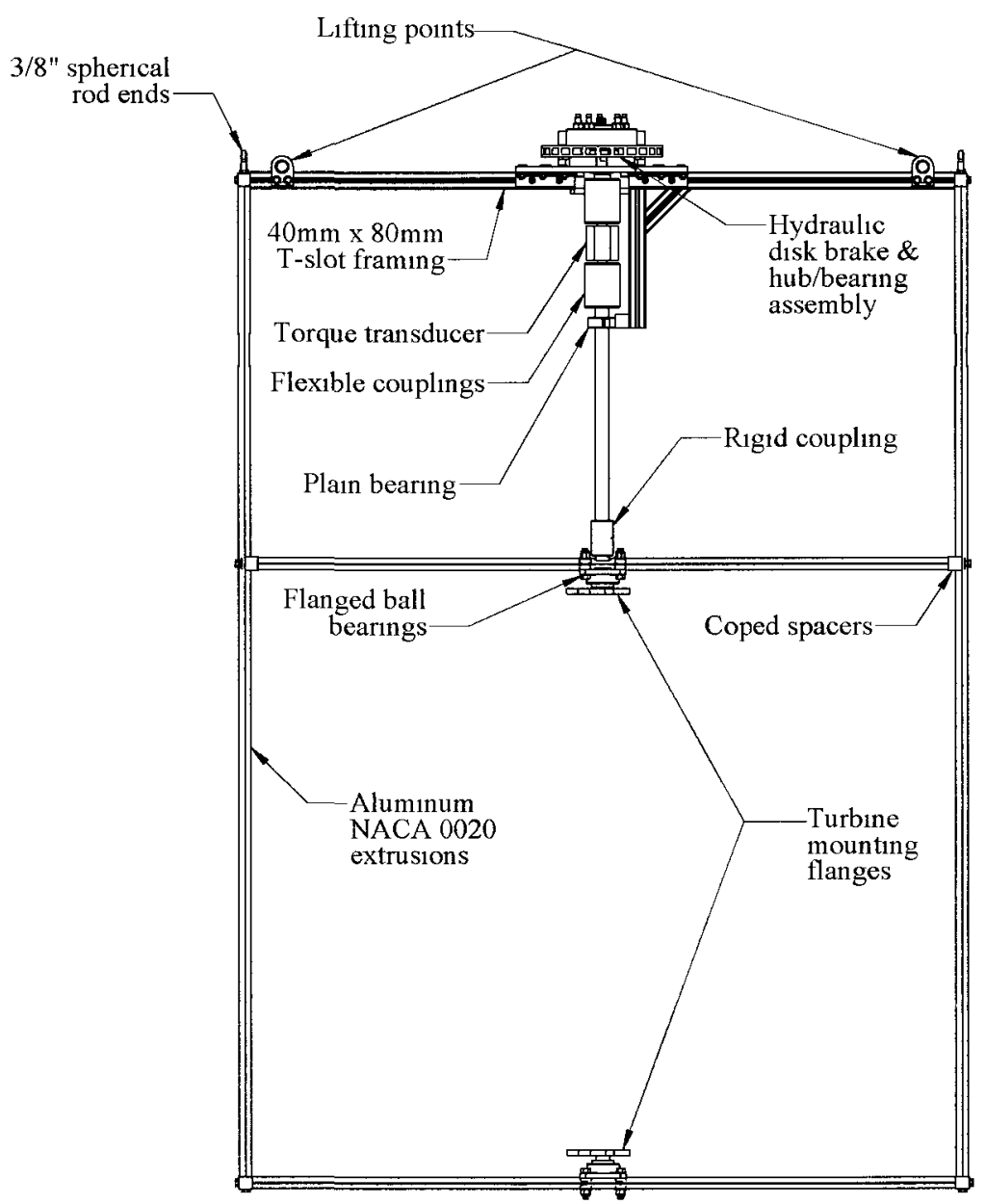

Figure 3.7 Main frame assembly.

Resistive torque is provided by a hydraulic disc brake above the torque transducer. Conveniently, the automotive industry manufactures integrated hub/bearings that include 
a toothed ring and magnetic pickup for sensing wheel speed, on which a brake rotor can be easily mounted. One such hub assembly was chosen for the uppermost bearing of the main frame assembly. The magnetic pickup (a Hall-effect device) provides a 54 pulseper-revolution signal that does not require excitation voltage, the frequency of which can be measured to calculate turbine shaft RPM. This bearing normally mates to an axle shaft via internal splines, so the male end of an axle shaft was modified to fit the hub on one side and a flexible coupler on the other. The flexible coupler halves that attach to the torque transducer were modified to make them clamp the shaft, since the torque transducer shaft has no keyway.

The brake master cylinder is a Wilwood 260-6766 with a 1 inch diameter bore and is connected to the brake caliper with a flexible braided stainless steel hose with -3 AN flared fittings on each end. A lever and bracket were fabricated to allow manual actuation of the brake. With a lever ratio of approximately 7 and an area ratio (between slave and master cylinders) of approximately 8 , the brake system can produce $200 \mathrm{Nm}$ of resistive torque with roughly $80 \mathrm{~N}$ of force applied at the lever, assuming a kinetic friction coefficient of 0.35 . A block, screw, and jam nut are attached to the master cylinder to allow the limiting of maximum brake force. A mechanical pressure gauge was installed to monitor brake fluid pressure, helping with load repeatability. The brake lever bracket assembly is designed to be clamped by four $1 / 4-20$ bolts and two flat bars to one of the 2 inch square members of the carriage, allowing the brake lever bracket assembly to be removed with the turbine frame subassembly, preventing the need for disconnecting the hydraulic line. 
The brake is mounted so its force points in the cross-stream direction; this way it will not affect drag measurements. A free body diagram is shown in Figure 3.8.

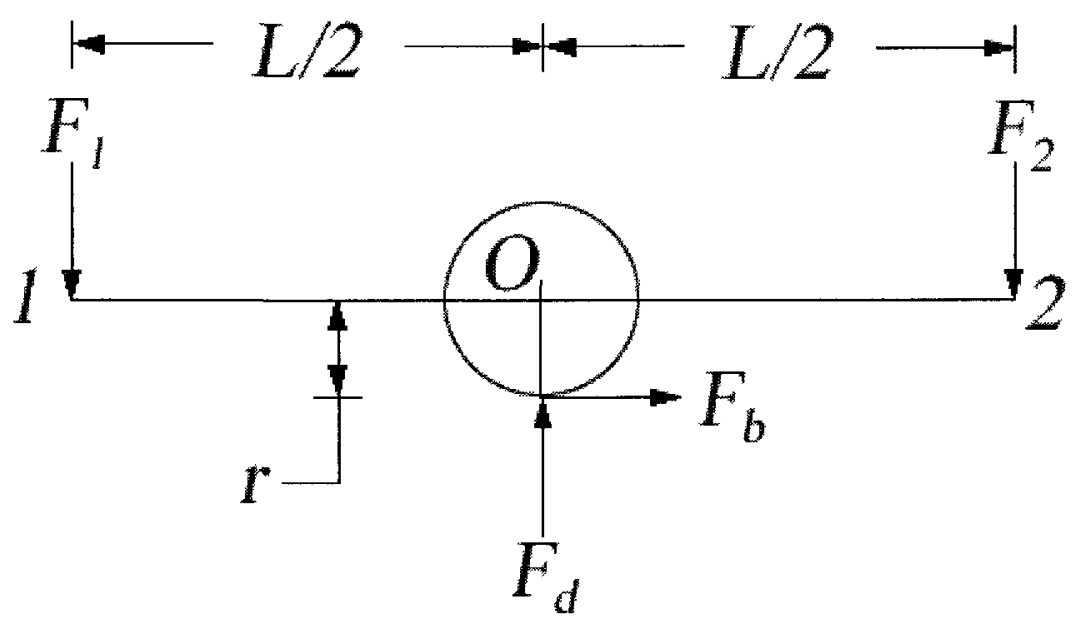

Figure 3.8 Free body diagram of loads on test frame.

From this free body diagram, it was determined that brake force would not affect total drag measurements, though it would affect each individual load cell's measurement. Summing forces in the vertical direction gives

$$
F_{1}+F_{2}=F_{d}
$$

While summing moments about point $O$ gives

$$
r F_{b}=L / 2\left(F_{2}-F_{1}\right)
$$

showing how brake force, and therefore torque, will affect each load cell's reading. 


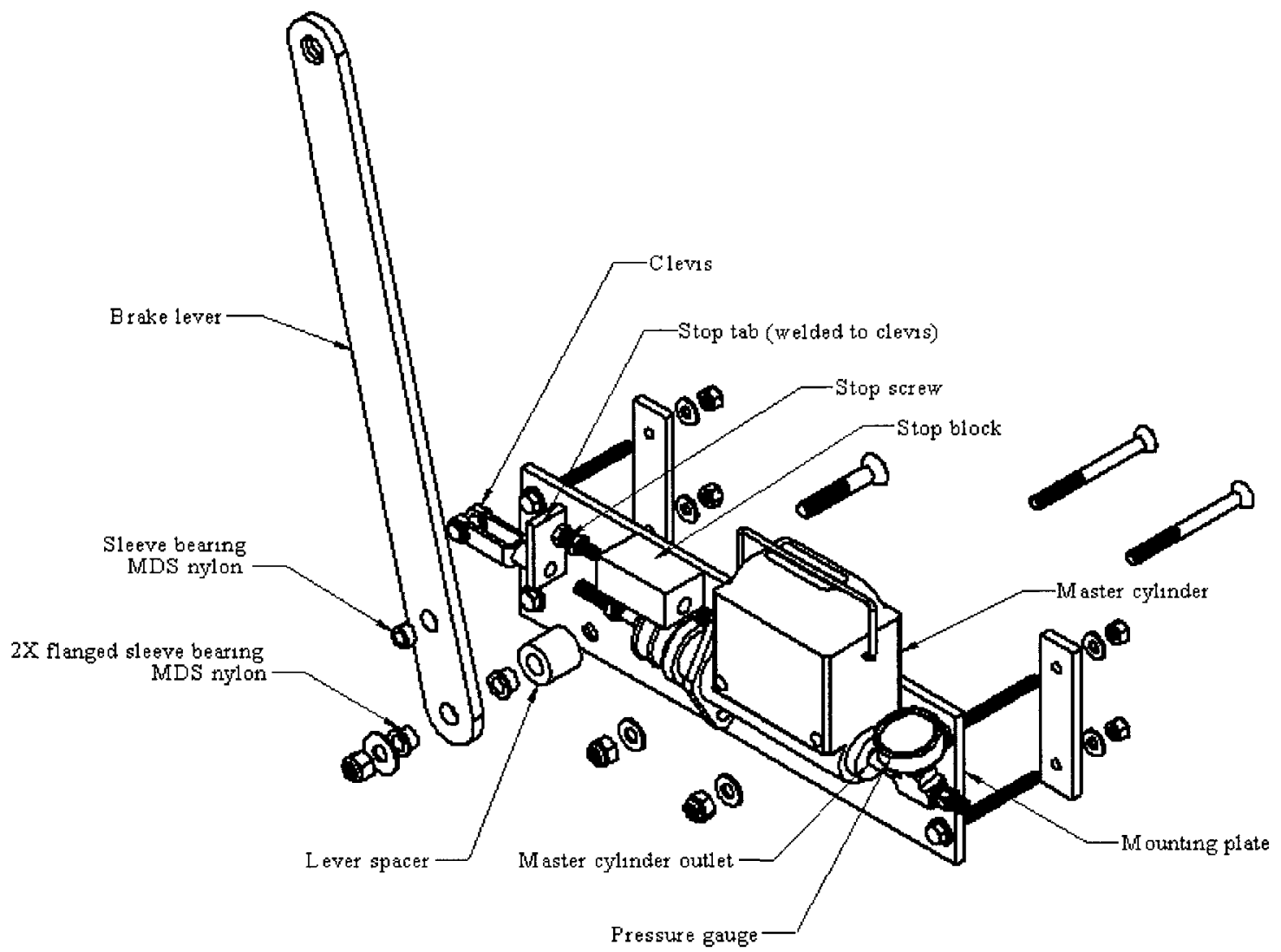

Figure 3.9 Exploded view of brake lever bracket assembly.

Most of the parts made from flat plate (6061-T6 aluminum and 316 stainless) were sent out to be machined with a water jet cutter to save time.

Regarding user friendliness, lifting points are located on the upper cross member of the turbine frame assembly to aid in removal from the tank. The frame was originally designed to be able to be flipped up about the horizontal axis of the main frame's spherical rod ends, for swapping out turbines. However, after some use it became apparent that it was easier to remove the entire turbine frame assembly and install the turbines outside of the tank. This process takes roughly one hour for two people to complete. 


\subsection{Drag Measurement - Rail Subassemblies}

The purpose of the two rail subassemblies, one shown in Figure 3.10, is to support the main frame, while allowing all streamwise force to be transferred to force measurement transducers. It was determined that the loading due to drag force on the turbine was low enough to allow the vertical foil struts of the main frame to be cantilevered below the water surface. This meant the rail subassemblies could attach to the main frame assembly right above the water surface, helping to further minimize drag.

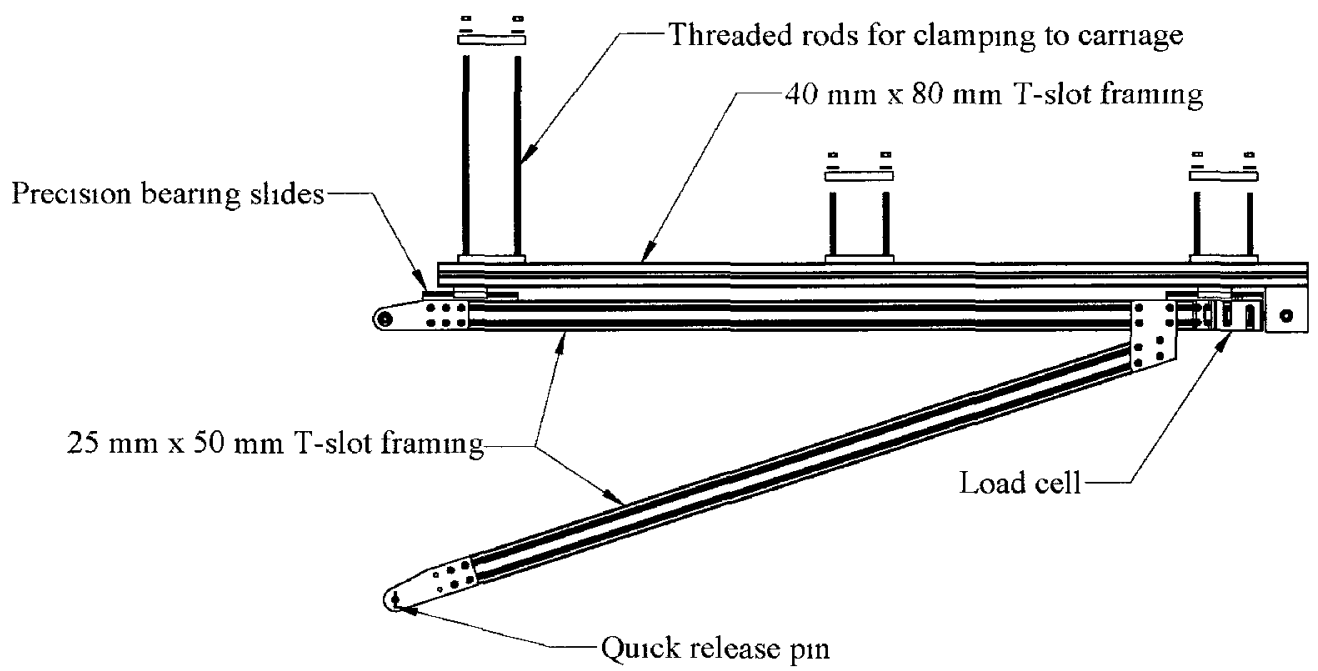

Figure 3.10 Rail subassembly.

The main frame attaches to each rail subassembly at two points per side. The uppermost point is a spherical rod end to allow pivoting upward along the cross-tank axis, which also helps eliminate binding from non-uniform streamwise loading, though deflection of the load cells is quite small even at maximum rated force.

In order to measure streamwise drag, the frame to which the turbine is mounted is free to move in the streamwise direction so load cells can measure the force required to hold it in place. Two precision bearing slides per side permit freedom of motion in this 
direction. These were chosen due to their load capacity and similarity of hole position (hardened material would be hard to modify) to the slots in 8020 framing. Custom T-slot nuts were fabricated to keep the rails in line with each other and to accommodate the smaller $4 \mathrm{~mm}$ screws used with the bearing slides. These small screws were a concern for strength since they support the entire weight of the test frame, though since there are 12 of them the calculated stress in each is lower than their yield strength. If the test frame was made heavier by a significant amount, more bearing slides could easily be added to increase load capacity.

Two Sentran ZB $500 \mathrm{lbf}$ capacity load cells were chosen for measuring drag force. They are rigidly mounted at one side and mounted by a spherical rod end at the other. Their combined capacities would allow the GHT to be towed up to approximately 2.6 $\mathrm{m} / \mathrm{s}$, though the design of the rails is such that swapping to larger capacity load cells would be extremely simple, since the form factor of the $500 \mathrm{lbf}$ cells is the same for cells up to $1500 \mathrm{lbf}$ capacity.

\subsection{Signal Conditioning and Data Acquisition}

Torque is measured by an Interface T8 $200 \mathrm{Nm}$ torque transducer mounted between the brake and turbine. The uppermost bearing features an integral 54 pulse-per-revolution magnetic pickup used to measure shaft angular speed, whose frequency is measured with

a Dataforth DSCA45 frequency signal conditioner. Load cell excitation voltage and signal conditioning are supplied by Dataforth DSCA38 strain gage signal conditioners. Carriage speed is measured by a 60 pulse-per-revolution encoder driven by a $4.8 \mathrm{~cm}$ diameter wheel, which rides along the main carriage rail. This encoder pulse frequency is 
measured with a Dataforth DSCA45 frequency signal conditioner. A National Instruments USB-6211 DAQ device is used to record all analog voltages. An attempt was made to use the counter inputs on the USB-6211 to record the frequencies of the tachometer and carriage speed measurement encoder directly. Unfortunately, there were not sufficient memory resources to accomplish this while simultaneously recording the other required analog voltages.

\subsection{Tare Drag and Torque}

In order to estimate only the streamwise drag on the turbine, the drag of the frame alone was measured to be subtracted later. Since towing the LST required two extra shaft extensions, its tare drag was measured separately with these installed. Tare drag in both configurations is plotted versus carriage velocity in Figure 3.11. The tare drag of the test bed is low, roughly 25 times lower than the measured turbine drag (for the GHT).

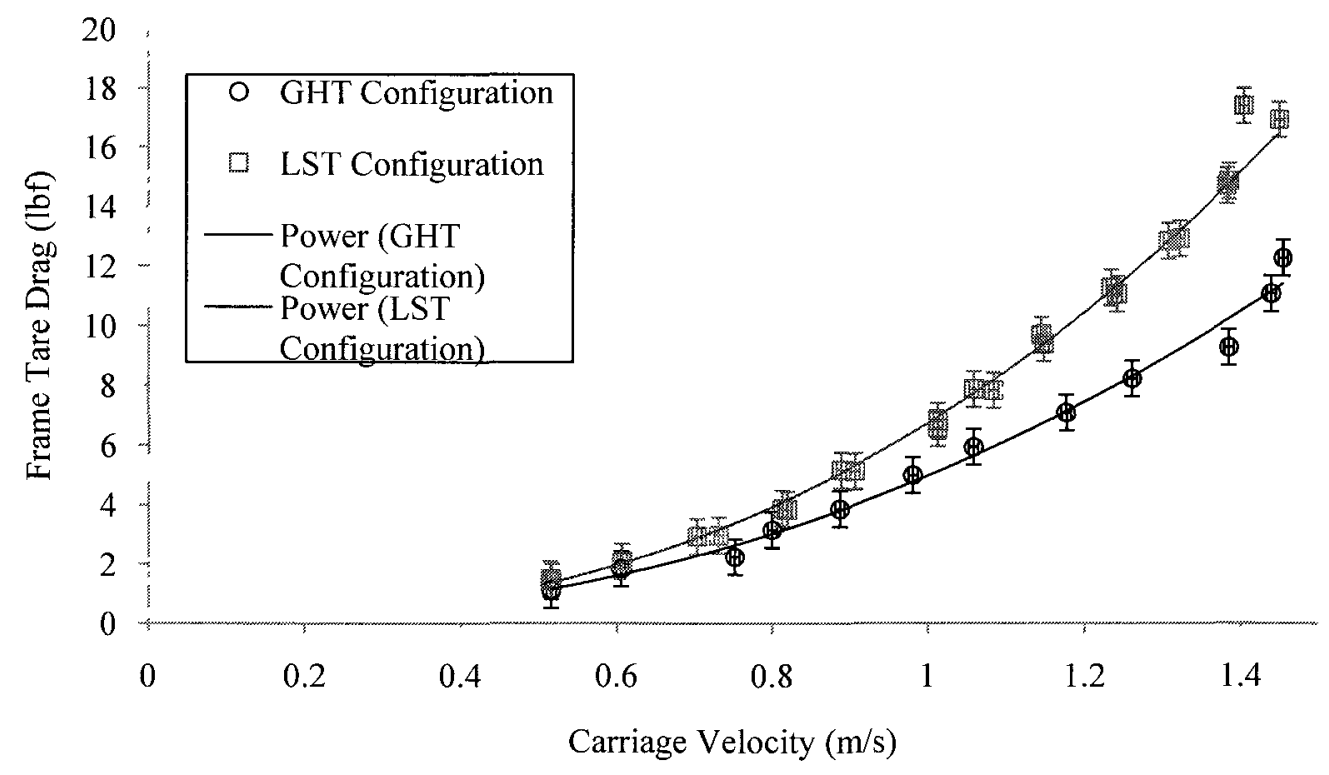

Figure 3.11 Frame tare drag versus carriage velocity. 
Similarly, to estimate the true hydrodynamic torque, friction in the bearings below the torque transducer was measured by rotating them in air, regressed linearly versus shaft rotational speed, and added to the measured value in post-processing. Figure 3.12 shows the measured tare torque plotted versus RPM for the shaft speed ascending in forward and reverse directions of rotation. The hysteresis observed was within the manufacturer's combined error specification $( \pm 0.5 \mathrm{Nm})$. It is assumed the ascending values will be a better estimation of the true tare torque, so a linear regression is used with these values.

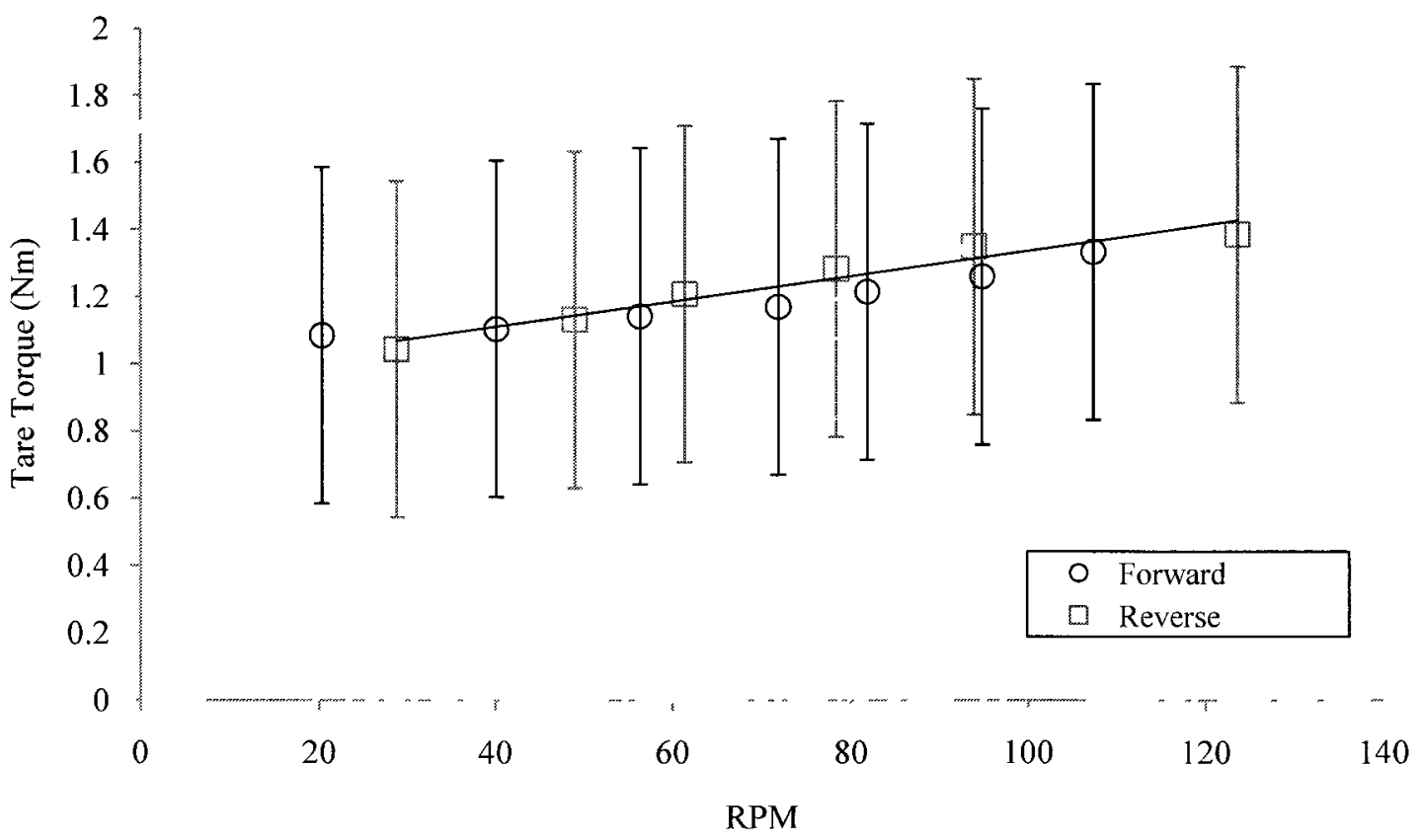

Figure 3.12 Tare torque versus RPM for forward and reverse directions with corresponding linear regression. 


\subsection{Final Test Bed Design Summary}

The test bed consists of four main assemblies: The main frame assembly, two rail subassemblies, and the brake lever bracket assembly, all of which are attached to the tow carriage. The entire test bed outline is shown in Figure 3.13 and photos of the test bed with the turbines installed are shown in Figure 3.14. Table 3.3 gives a summary of the test bed characteristics, including the power law fits for tare drag and linear fit for tare torque with ascending RPM. 


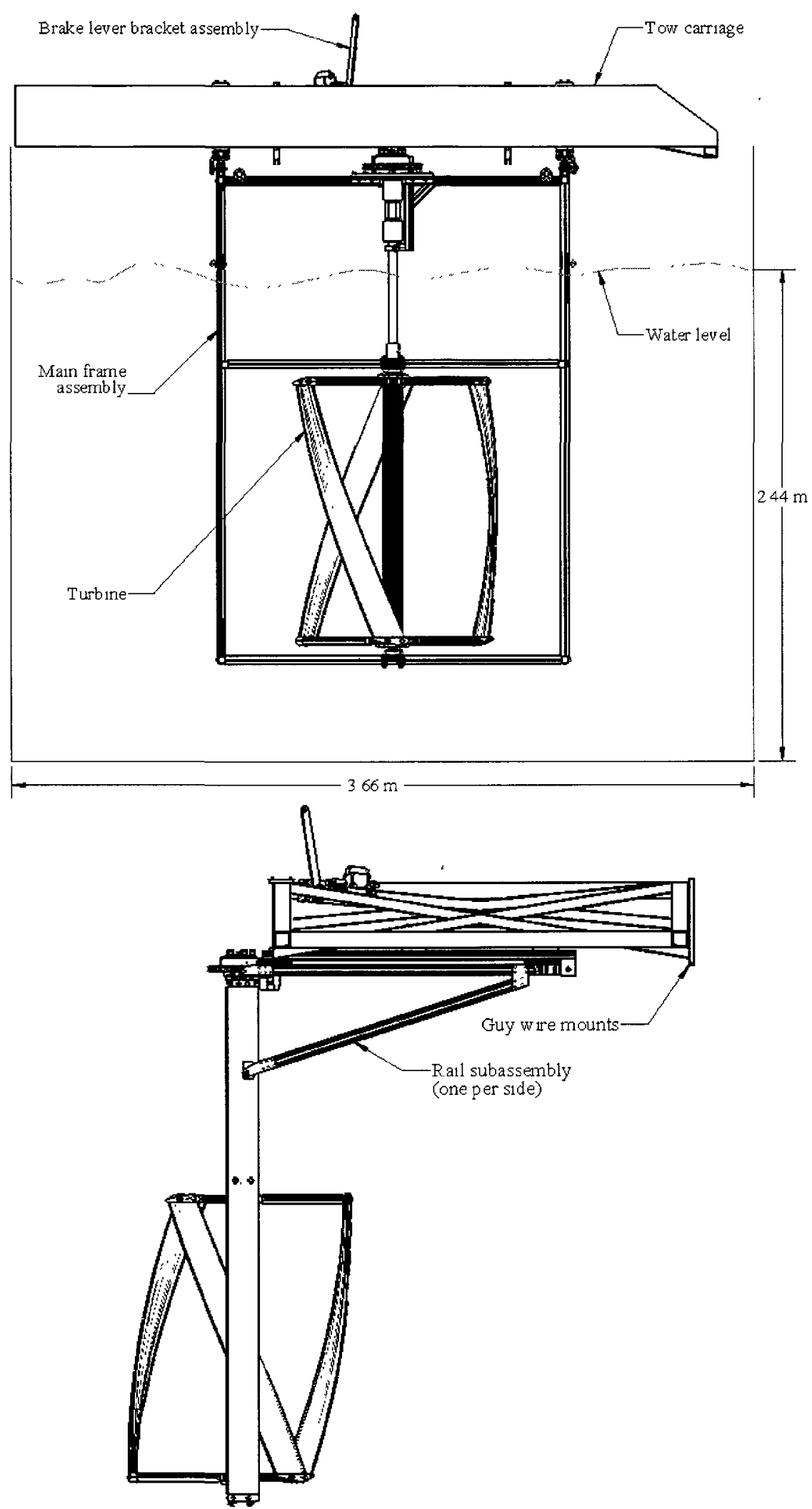

Figure 3.13 UNH-CORE hydrokinetic turbine test bed outline. 


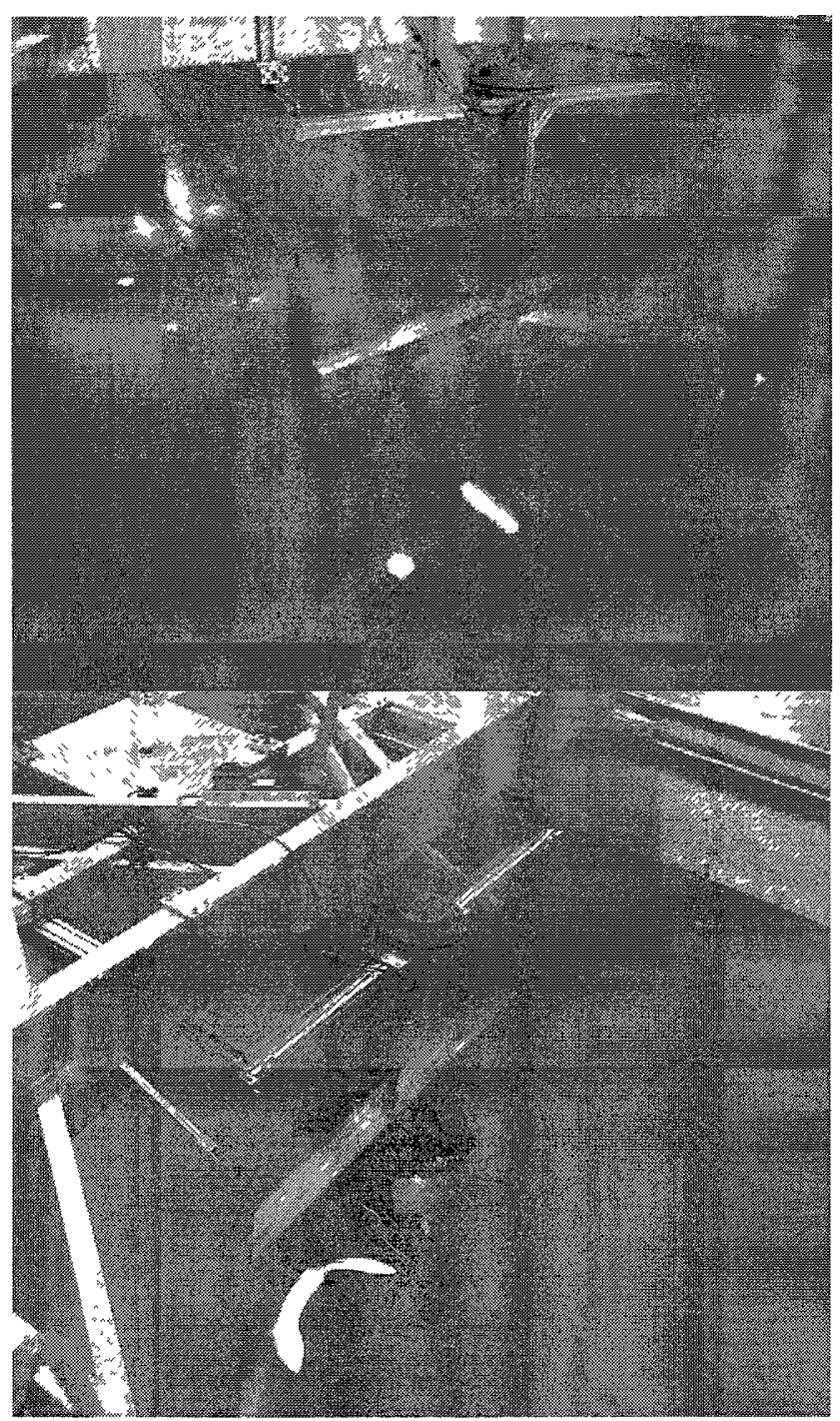

Figure 3.14 GHT (top) and LST (bottom) installed in the UNH-CORE hydrokinetic turbine test bed.

Table 3.3 Turbine test bed characteristics as built.

\begin{tabular}{cc}
\hline Max. turbine height & $1.32 \mathrm{~m}$ \\
Max. turbine diameter & $1.5 \mathrm{~m}$ \\
Max. turbine frontal area & $1.3 \mathrm{~m}^{2}$ (up to $1.5 \mathrm{~m} / \mathrm{s}$ ) \\
Max. shaft torque & $200 \mathrm{Nm}$ (transducer limit) \\
Max. drag force & 1000 lbf (load cell limit), 507 lbf \\
& (tow mechanism limit) \\
Tare drag estimate & $F_{\text {tare }}=4.98 U^{221}$ for GHT; \\
& $F_{\text {tare }}=6.74 U^{241}$ with LST \\
& extensions \\
Tare torque estimate & $T_{\text {tare }}=0.0038 R P M+0.9599$ \\
\hline
\end{tabular}




\subsection{Add-ons: Turbulence Generators}

In order to generate turbulence, various objects can be placed upstream of the turbine. Two types of turbulence were desired for this study, grid (isotropic homogeneous) turbulence and the turbulent wake of a cylinder with diameter on order of the turbine blade chord length. To generate grid turbulence, a grid was designed and fabricated from 0.5 inch diameter steel re-bar with a 2.5 inch mesh width, resulting in a 0.36 solidity, shown in Figure 3.15. This 0.36 solidity is the lowest that will produce isotropic homogeneous turbulence (Groth \& Johansson, 1988), but kept drag to a minimum to conserve tow power. The turbulence generators are designed to mount in the inner streamwise slots of the larger T-slot framing of the rail subassemblies, independent of drag measurement. This mounting configuration allows varying streamwise position to some extent. Similarly a cross-member was fabricated from T-slot framing that allows the mounting of a cylinder at various streamwise or cross-stream locations, shown in Figure 3.16. Both the grid and cylinder are kept vertical with guy wires running to the front of the carriage. 


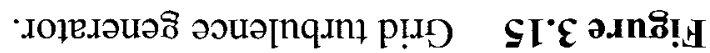

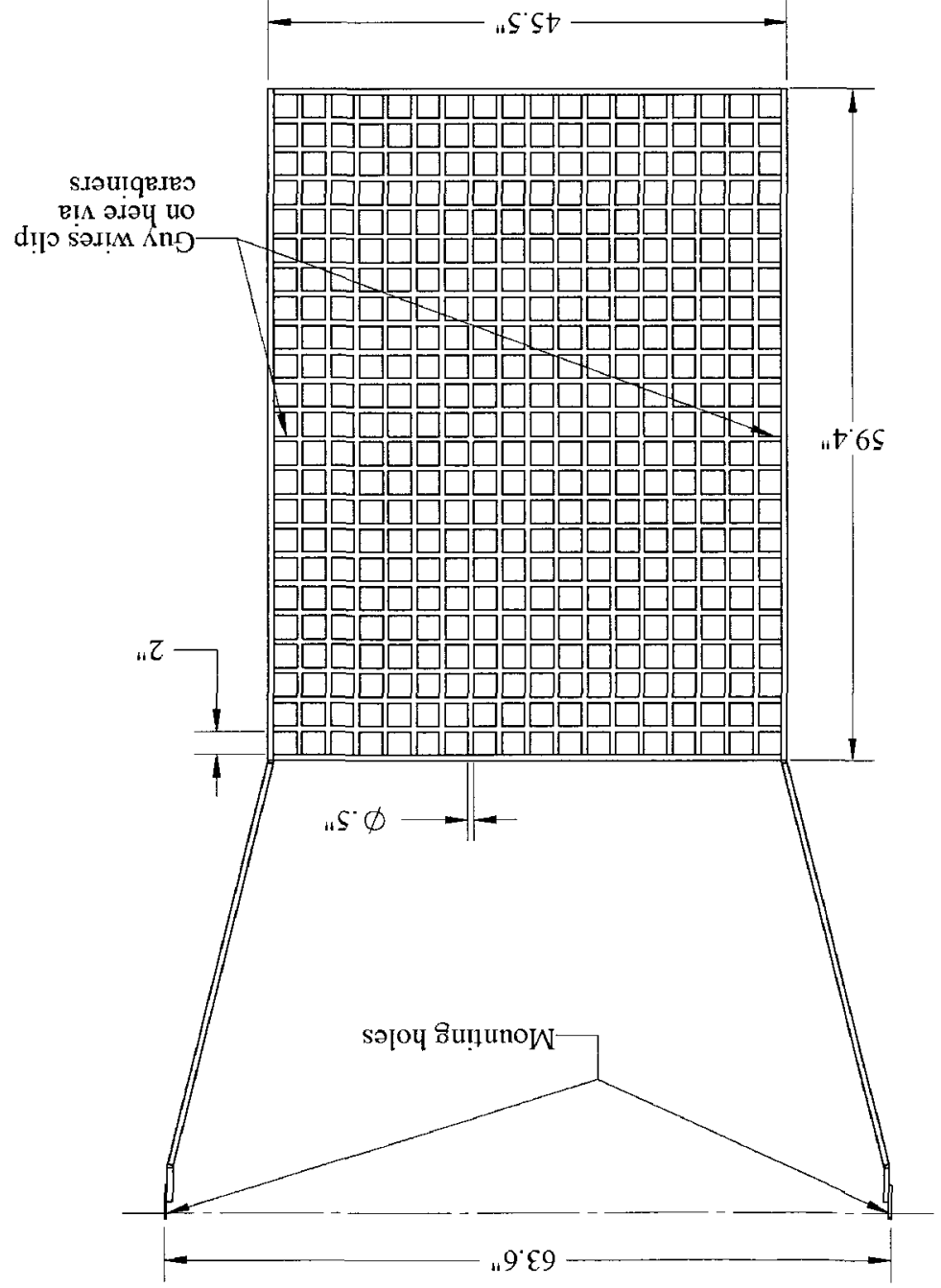




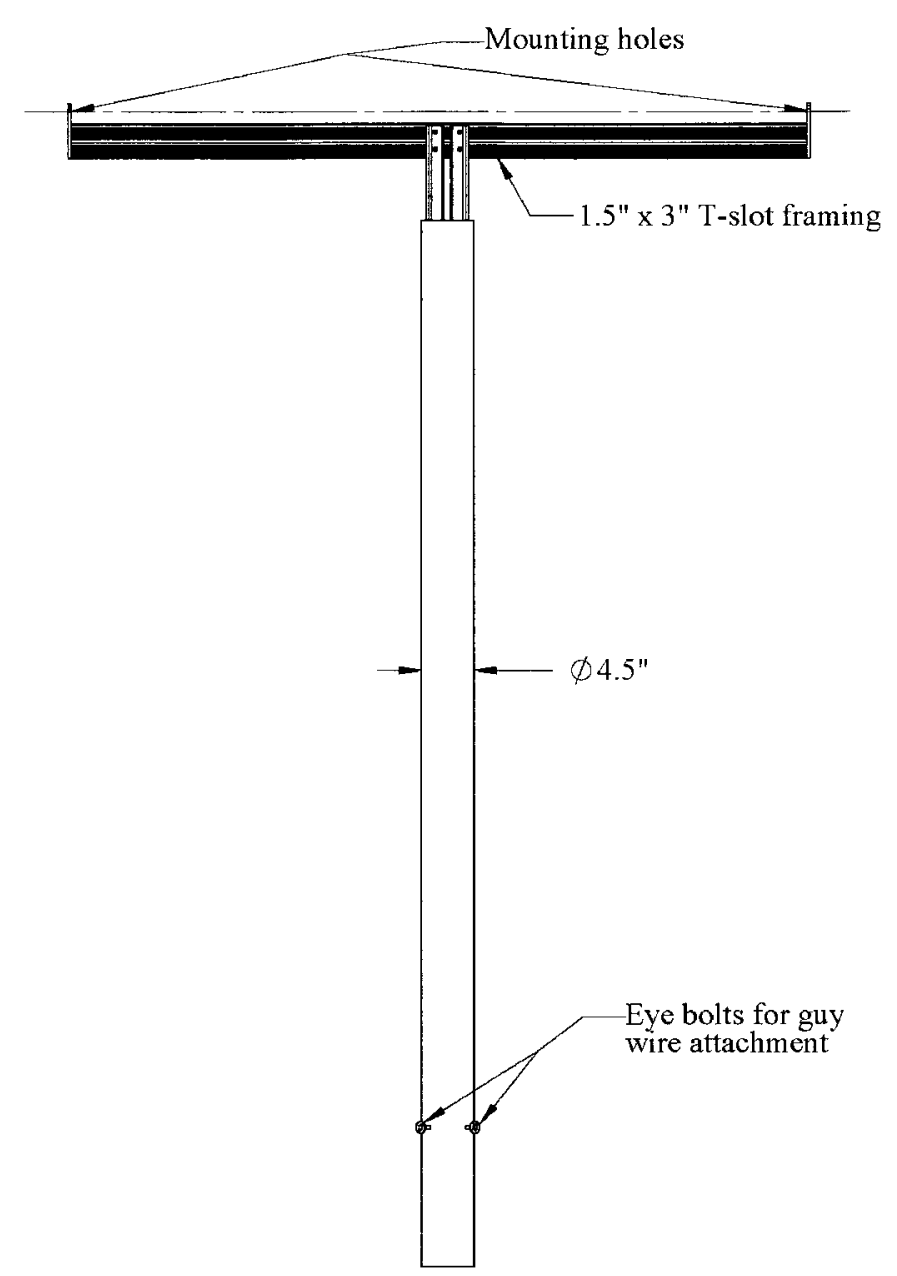

Figure 3.16 Cylinder wake turbulence generator.

\subsection{Add-ons: Flow Measurement Devices}

Fluid velocity can be measured in a number of ways. One is a simple Pitot-static tube with a pressure transducer. This method works well for mean velocities but is not appropriate for measurements of high frequency fluctuations such as those in turbulence. Flow in water can be measured using acoustic Doppler techniques, using devices that calculate fluid velocity from the Doppler shift of sound waves scattered off particles moving with the water. The Doppler shift is the change in frequency of a scattered sound due to relative velocity of the scatterer. These devices fall into two major categories, acoustic Doppler current meters or profilers (ACMs or ADCPs) and acoustic Doppler 
velocimeters (ADVs). ADCPs measure velocity by emitting one or more diverging beams, then "listening" for the scattered signal. By temporally gating the return signal it can be divided into "bins" which each correspond to a volume at a certain mean distance from the instrument. The ADCP measurement assumes isotropy and homogeneity along the diverging beams. ADVs measure velocity inside a much smaller volume and at a much higher sample rate than ACMs or ADCPSs. In general, an ADV provides a "quasipoint" measurement (measurement volume on the order of $1 \mathrm{~cm}^{3}$ with temporal resolution in the $100 \mathrm{~s}$ of $\mathrm{Hz}$ ), whereas the spatial averaging along diverging beams of the ADCP measurements results in a measurement volume on the order of $(10 \mathrm{~cm})^{3}$ or larger with a temporal resolution on the order of $1 \mathrm{~Hz}$. Since this study would require measuring velocity in grid turbulence an $\mathrm{ADV}$ was chosen as the appropriate flow measurement device for these experiments. A Nortek-AS Vectrino+ in particular can measure velocity at sample rates up to $200 \mathrm{~Hz}$.

Bracketry for mounting the ADV was designed and fabricated. The main ADV mounting structure is a piece of 1 inch $\times 3$ inch T-slot framing. Brackets were designed and fabricated to clamp this strut to one of the 3 inch square members of the tow carriage, allowing for cross-tank and vertical adjustment of probe position. The ADV itself is clamped to the strut with two sets of HDPE straps. A similar mounting structure was designed and fabricated for mounting a Nortek-AS Aquadopp ACM, though this device was not used in the later experiments reported here. 


\section{Turbine Performance in Steady Uniform Flow}

\subsection{Data Collection Procedure}

At first, it was assumed the turbine could be allowed to self start. This became a problem due to the rapid increase in drag force as the turbine begins to rotate. This change in drag force applied a shock load to the towing mechanism and lead to large oscillations. These oscillations are present even when simply towing an unloaded carriage, but the turbine certainly exacerbated the problem. Darnell took note of this low frequency resonance in 1996, but to date no measures have been taken to reduce it. Due to the lack of damping in the system, the carriage will oscillate. More details about this issue are discussed in Appendix B.

To avoid these unsteady forces and subsequent oscillations, the turbine was started manually before starting each tow. Once the carriage has almost fully accelerated the brake is applied until the lever hits the stop, approximating a step input of loading. Once this load torque is applied, turbine shaft RPM decreases until reaching a steady state value. All signals were sampled at $500 \mathrm{~Hz}$ via a VI (Virtual Instrument) programmed in LabVIEW.

Figure 4.1 shows a sample data collection run (in arbitrary units, after applying a 500 sample wide moving average smoothing filter in MATLAB) for one tow to illustrate how measurements vary with each other and to show a typical steady state duration of interest, on the order of a few seconds. Measurements were averaged over this steady state duration, producing one operating data point for the turbine under tow. Note that the very beginning of the run shows the turbine being started manually, hence the negative 
torque readings. In addition to reducing transient drag forces explained earlier, manual starting helped the turbine reach steady state more quickly, since tank length is limited.

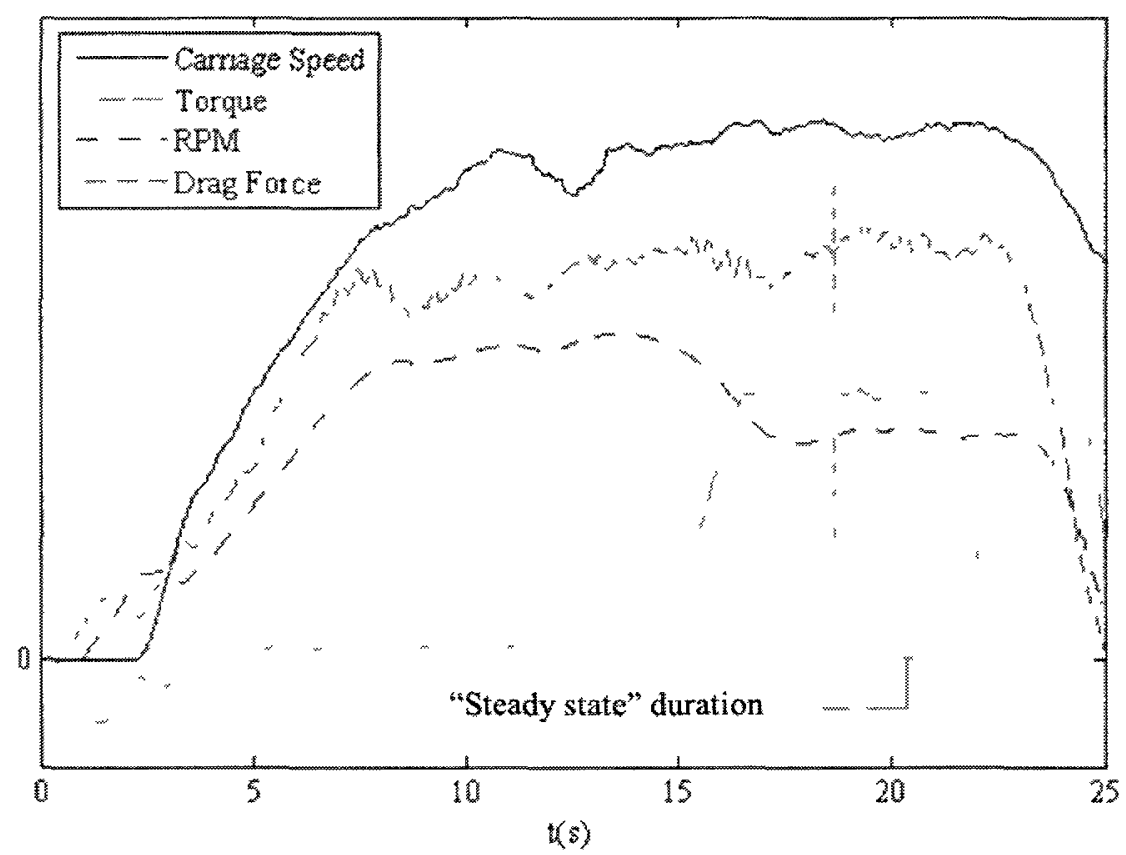

Figure 4.1 Sample data collection time series towing the GHT at $U=1 \mathrm{~m} / \mathrm{s}$ showing carriage speed, turbine shaft RPM, torque, and overall drag force (in arbitrary units).

Each day during testing, a sample of water was taken from the tank and its density was measured with a hydrometer. The water level in the tank was also measured with a tape measure, for calculating the tank cross-sectional area used in the blockage corrections.

\subsection{Data Reduction and Uncertainty Estimates}

As with all experimental research, it is important to fully document the measurement and data reduction methods used and provide best estimates of experimental uncertainty. Factory calibrations for the load cells and torque transducer were used for data 
processing. The Dataforth signal conditioners were also used with their factory calibrations. See Appendix A for calibration sheets.

The drag on the device is assumed to be zero when the carriage is not moving, so the average measurement of the drag signal for the first few seconds of each run is subtracted as the zero load value. This compensates for any long term zero drift and in effect zeroes/tares the system for each run.

Error is estimated using the manufacturer's "combined error" specifications from their respective datasheets, which are higher than the values from their provided calibration sheets, serving as a conservative estimate. Errors for each individual component considered are tabulated in Appendix A. The experimental error for a parameter calculated from multiple measured quantities is calculated using the general formula for error propagation

$$
\delta q=\sqrt{\left(\frac{\partial q}{\partial x} \delta x\right)^{2}+\ldots+\left(\frac{\partial q}{\partial z} \delta z\right)^{2}},
$$

where $q$ is any function of the variables $x$ through $z$ (Taylor, 1997). Tip speed ratio, drag coefficient, and power coefficient are examples of variables calculated from several measurements. Experimental error estimates are summarized in Table 4.1. 
Table 4.1 Summary of experimental error estimates.

\begin{tabular}{|c|c|}
\hline Quantity, $q$ & Error, $\delta q$ \\
\hline Carriage Speed, $U(\mathrm{~m} / \mathrm{s})$ & $\sqrt{7.9 \times 10^{-8} U^{2}+4.2 \times 10^{-5}}$ \\
\hline Torque, $T(\mathrm{Nm})$ & 1 \\
\hline RPM & 0.6 \\
\hline $\begin{array}{l}\text { Drag, } F \text { (both sides, tare } \\
\text { subtracted, lbf) }\end{array}$ & $\sqrt{3.6 \times 10^{-7} F^{2}+0.20+\left(11 U^{12} \delta U\right)^{2}}$ \\
\hline Tip speed ratio, $\lambda$ & $\sqrt{\left(0.0001 \frac{R P M}{U}\right)^{2}+\left(0.05 \frac{R P M}{U^{2}} \delta U\right)^{2}+\left(\frac{0.03}{U}\right)^{2}}$ \\
\hline Power coefficient, $C_{\mathrm{p}}$ & 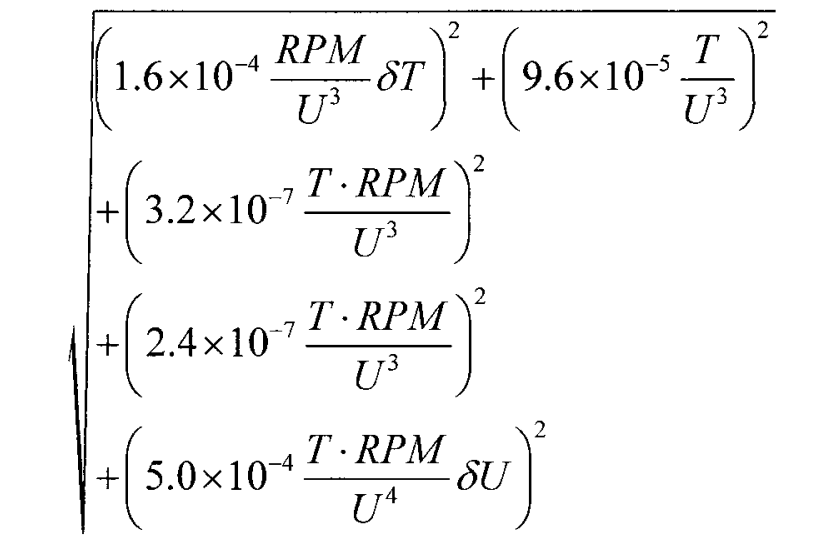 \\
\hline Drag coefficient, $C_{\mathrm{d}}$ & $\sqrt{\sqrt{\left.\frac{0.002}{U^{2}} \delta F\right)^{2}+\left(4.0 \times 10^{-6} \frac{F}{U^{2}}\right)^{2}}}$ \\
\hline
\end{tabular}

After applying instrument calibrations, data for each run was examined and the time values of the boundaries of its steady state duration (if it was reached) were determined. These times are stored in a ".mat" file so all runs can be processed by running one MATLAB script. This script then calculated all the relevant values for each run, averaged over the steady state time window.

Kinetic exergy efficiency was calculated with the measured power and drag coefficients. The induction factor used in the calculation was computed using the measured power coefficient and the momentum theory relation described in Equation (2.16). If one-dimensional momentum theory can be applied, this value would be on the 
right of the value corresponding to peak power coefficient, or greater than $1 / 3$. This makes sense if drag coefficients are greater than 0.8 (Manwell, McGowan, \& Rogers, 2002), which they are for these experiments.

\subsection{Results - GHT in Steady Flow}

Once the steady data from each run is extracted and processed, the measured power is normalized by the available kinetic power flux in the free stream through an area the size of the turbine's frontal area to calculate the power coefficient as described in Chapter 2. Figure 4.2 shows the GHT power coefficient plotted versus tip speed ratio before the blockage correction is applied. Figure 4.3 shows these same values after applying the blockage correction described in Section 2.2. Overall there was not a significant change observed in power coefficients at different free stream velocities. However, one result of interest is the significant increase in performance when carriage speed was increased from $0.8 \mathrm{~m} / \mathrm{s}$ to $0.9 \mathrm{~m} / \mathrm{s}$. It should be investigated further as to whether this is experimental error or a real consequence of increasing Reynolds number, which leads to higher stall angles, improving foil performance. A maximum blockage-corrected power coefficient of $28 \%$ was observed at a tip speed ratio of 2.1. Before blockage correction, a maximum power coefficient of $36 \%$ was reached at a tip speed ratio of 2.3 . This power coefficient is closer to the $35 \%$ reported by Gorlov (Gorlov, Development of the Helical Reaction Hydraulic Turbine, 1998). 


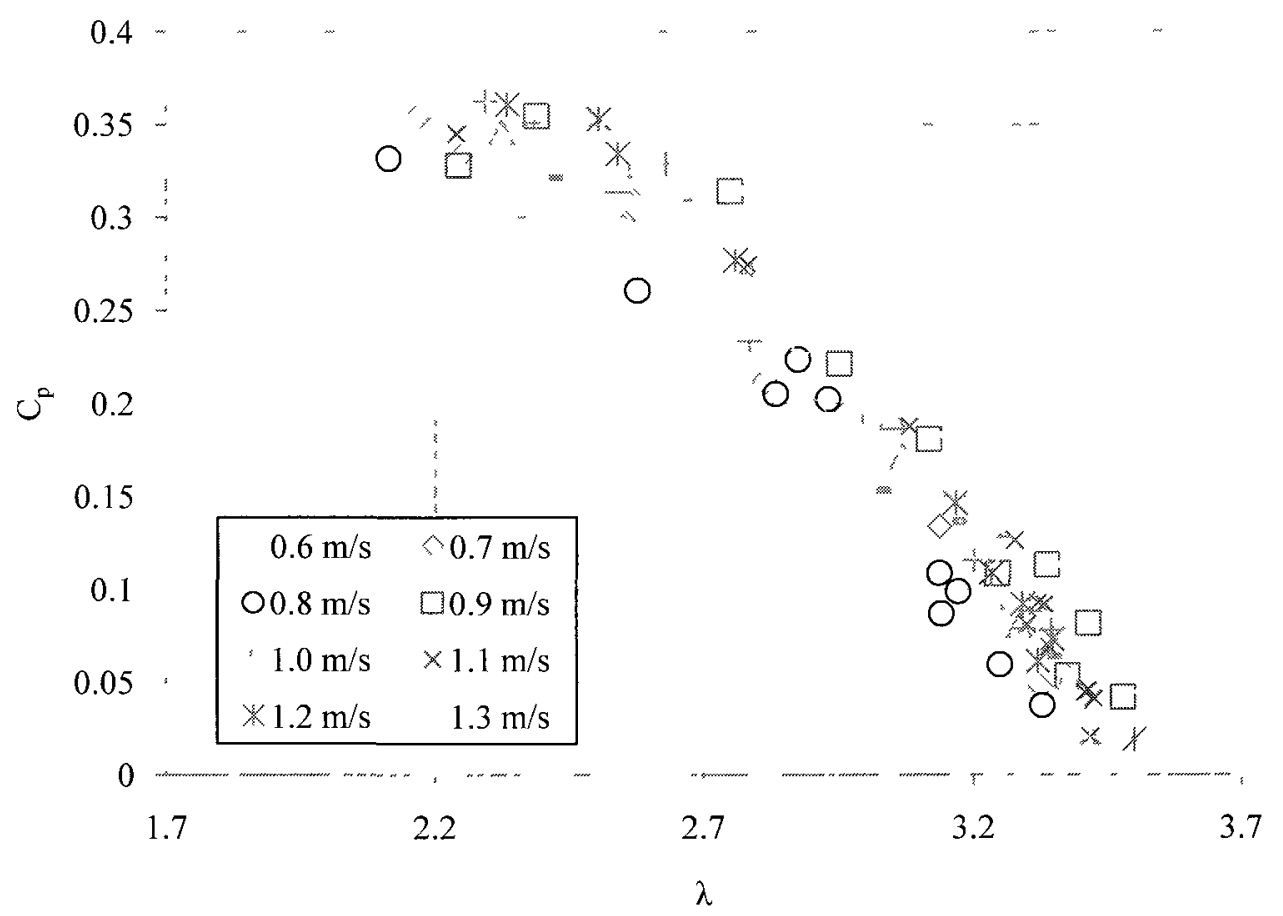

Figure 4.2 GHT power coefficient versus tip speed ratio without blockage correction. Average error for $C_{p}=0.02$; for $\lambda=0.04$.

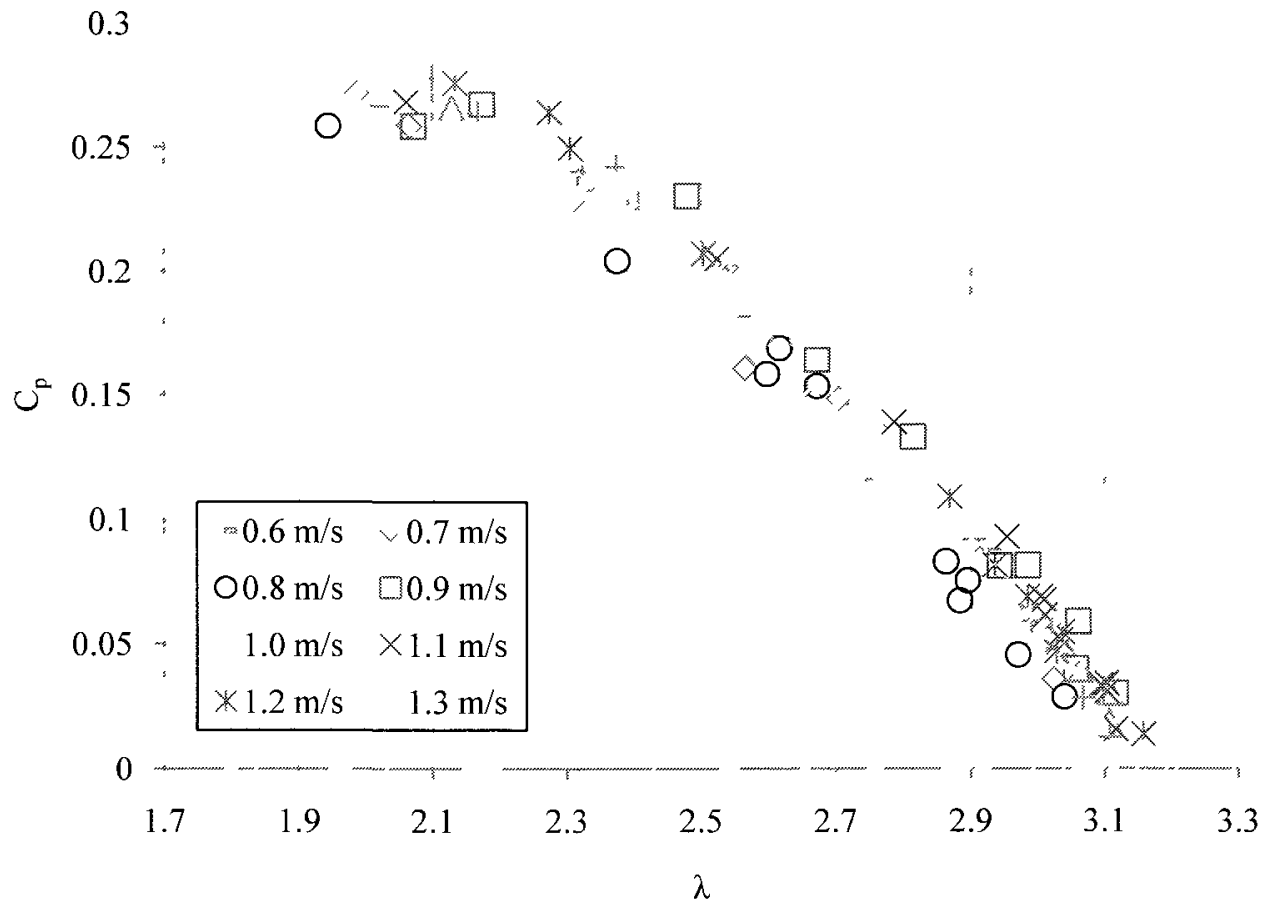

Figure 4.3 Blockage-corrected GHT power coefficient versus blockage-corrected tip speed ratio. 
Similar to the calculation of power coefficient, the measured drag is normalized using the drag coefficient defined in Chapter 2. Figure 4.4 shows GHT drag coefficient versus tip speed ratio without blockage correction. A slight increase in drag coefficients for higher tip speed ratios was observed. The values remain quite constant, slightly increasing as tip speed ratio increases. This makes sense since increasing tip speed ratio makes the turbine appear more and more like a solid body. In other words, the likelihood of a fluid particle passing through the device without a reduction in its momentum decreases. It is interesting that the drag on the device remained fairly constant regardless of the power taken out at the shaft. This is useful information for designing mounting structures and predicting environmental effects of these devices since it implies removal of momentum from the flow whether or not useful shaft power is extracted. Figure 4.5 shows blockage-corrected GHT drag coefficient plotted versus blockage-corrected tip speed ratio. All values are close to but do not exceed unity.

In 2000, Shiono et. al. performed an experimental study on a similar Darrieus turbine in a water channel. Peak power coefficient of $23.5 \%$ was achieved at a tip speed ratio of 1.82 and a solidity of 0.179 . Their peak power coefficient for a solidity of 0.366 occurred at a free stream speed of $0.8 \mathrm{~m} / \mathrm{s}$, with a significant decrease for a free stream speed of $1.4 \mathrm{~m} / \mathrm{s}$ (Shiono, Suzuki, \& Kiho, 2000). This could be a similar phenomenon as the reduced power for the GHT at $0.8 \mathrm{~m} / \mathrm{s}$. 


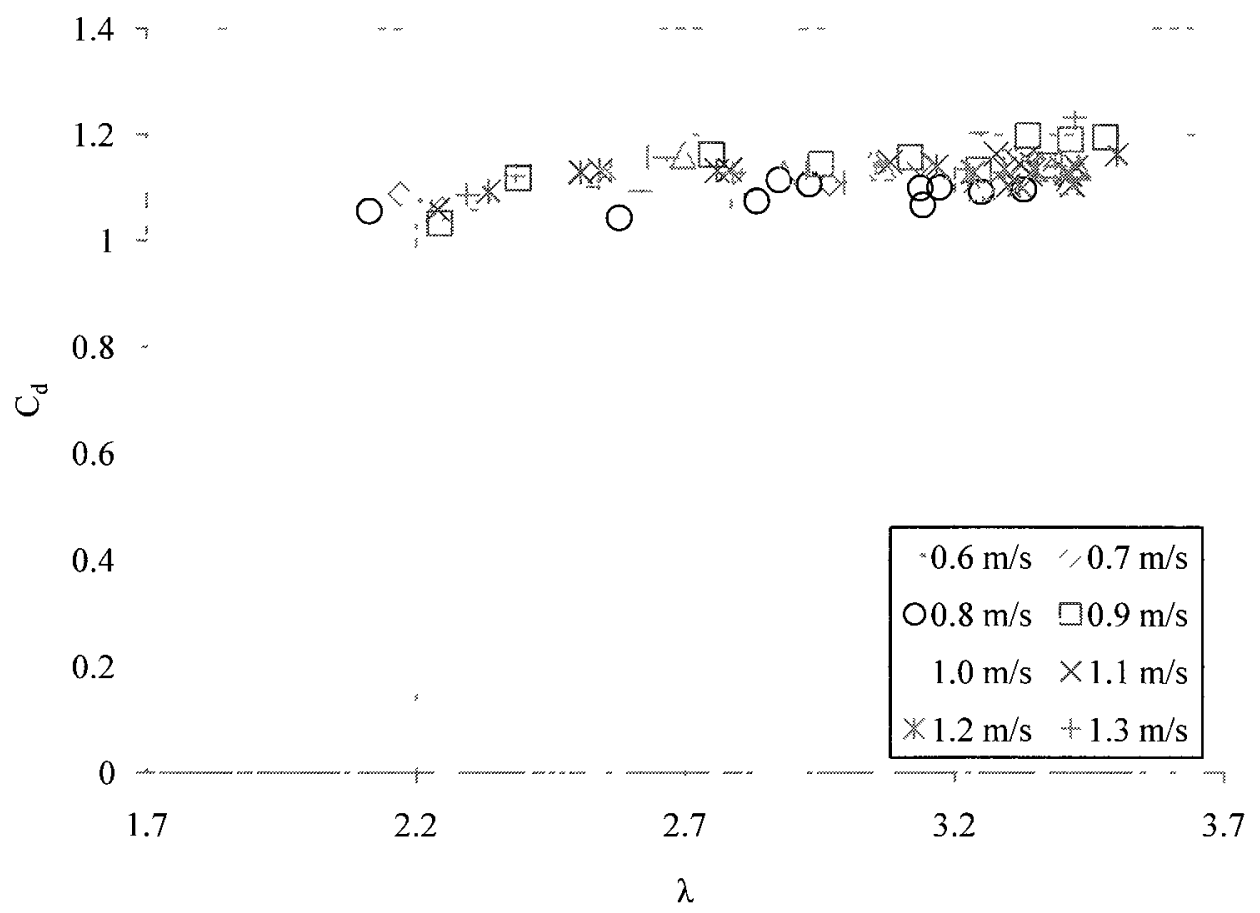

Figure 4.4 GHT drag coefficient versus tip speed ratio without blockage correction. Average error for $\mathrm{Cd}=0.004$; for $\lambda=0.04$.

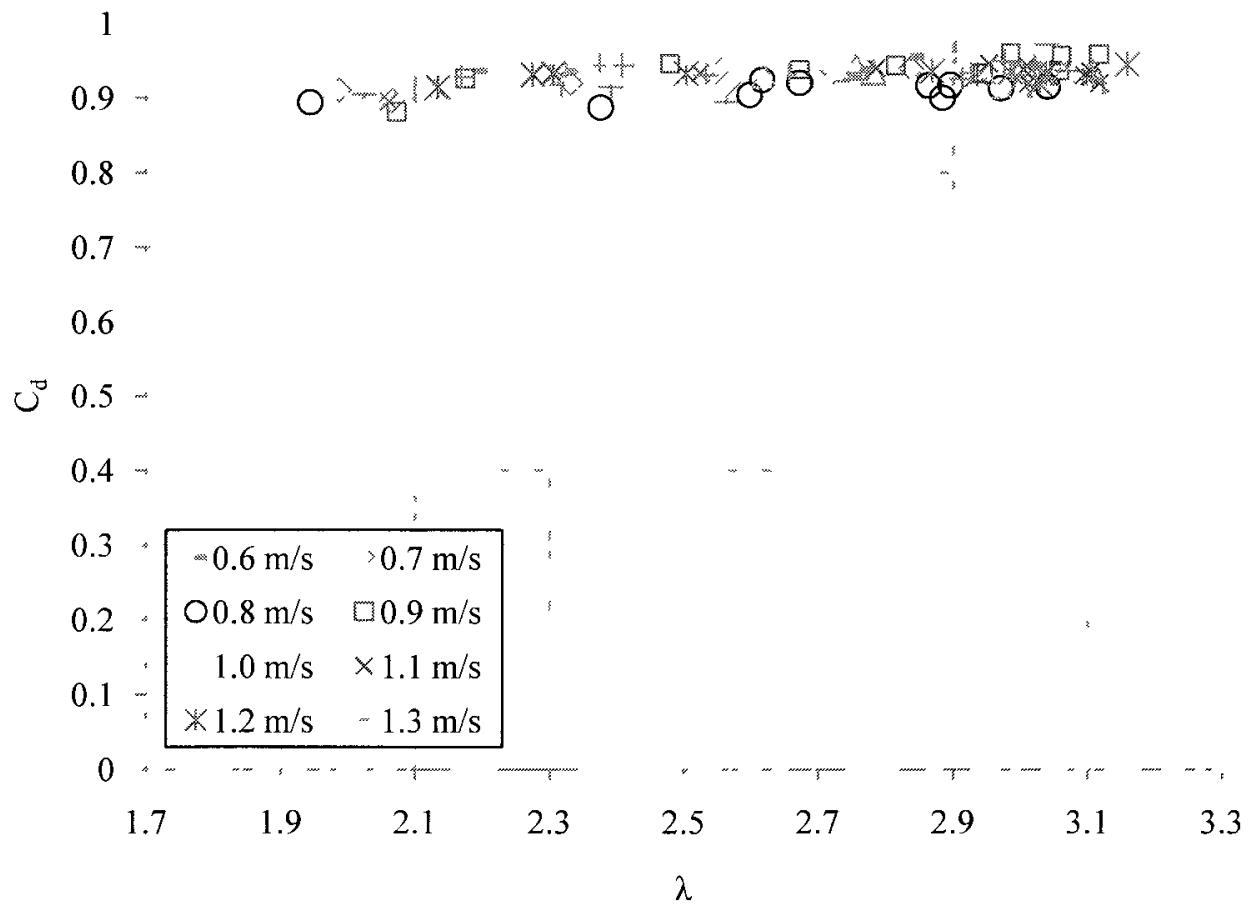

Figure 4.5 Blockage-corrected GHT drag coefficient versus blockage-corrected tip speed ratio. 
Figure 4.6 shows GHT kinetic exergy efficiency versus tip speed ratio (calculated using non-blockage-corrected power and drag coefficients). The device reaches a peak kinetic exergy efficiency of $87 \%$ at a tip speed ratio of 2.2 . It is remarkable how the kinetic exergy efficiency collapses more to a single curve at lower tip speed ratios compared to the power and drag coefficients.

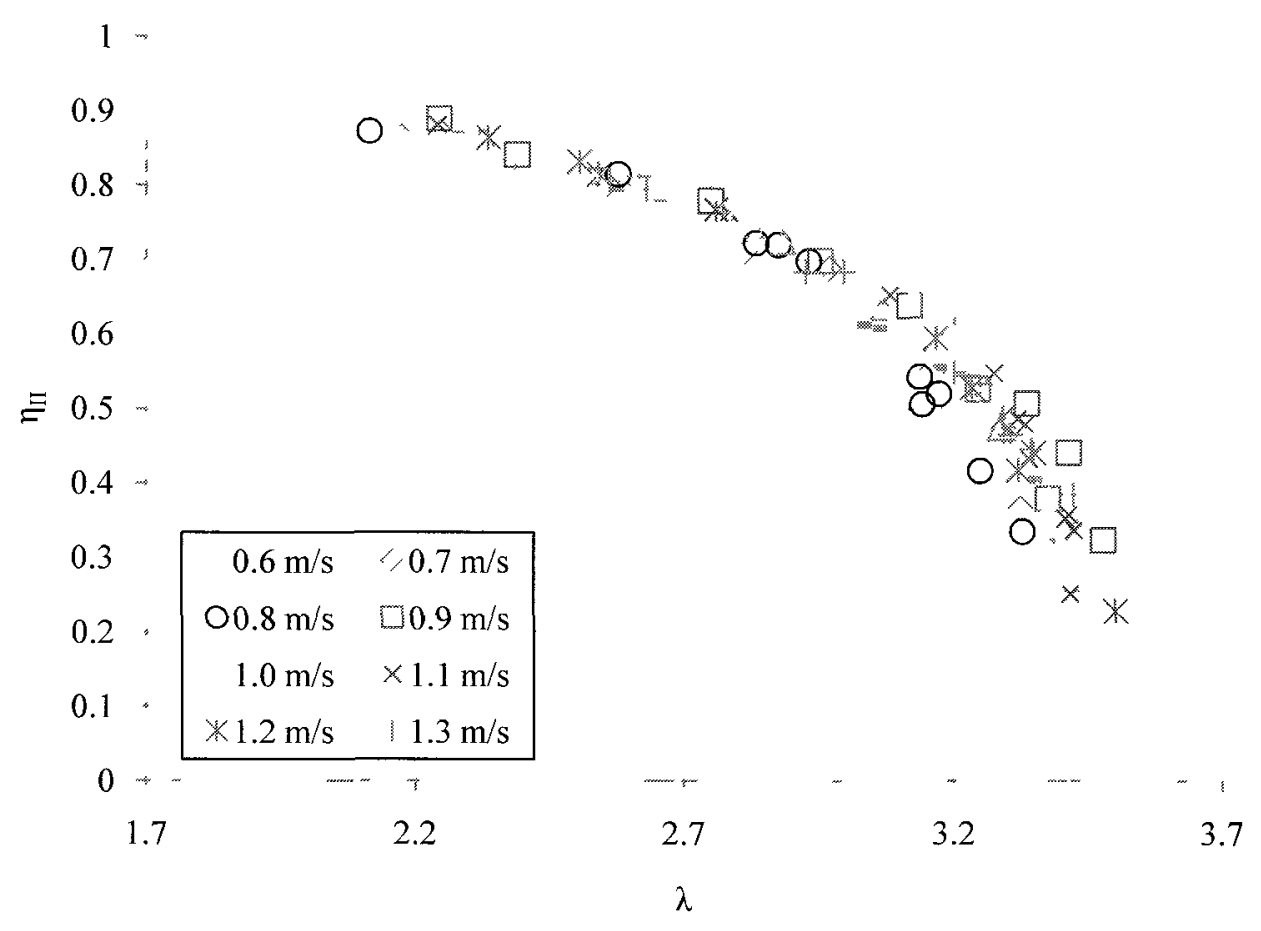

Figure 4.6 GHT kinetic exergy efficiency versus tip speed ratio.

\subsection{Results - LST in Steady Flow}

Figure 4.7 shows LST power coefficient versus tip speed ratio without blockage corrections. Compared with the GHT, power coefficients are lower and the device tends to operate at lower tip speed ratios. Low power can be explained by the device's circular cross section placing blade sections farther from the equator closer to the axis of rotation, therefore lowering local tip speed ratio, increasing local angle of attack oscillation 
amplitude, and inducing stall earlier at these blade sections. These blade section' lower radii also mean that blade forces impart a lower moment with respect to the turbine axis. Tendency to operate at lower tip speed ratios can be attributed to the device's higher solidity, consistent with findings on the operation of the Darrieus wind turbine (Paraschivoiu, 2002). Note that the power conversion efficiency of the LST has been measured at up to $46 \%$ when installed in a closed conduit of $4 \mathrm{ft}(1.22 \mathrm{~m})$ diameter (Schlabach R. , 2010). This flow configuration will change the induction characteristics and therefore performance.

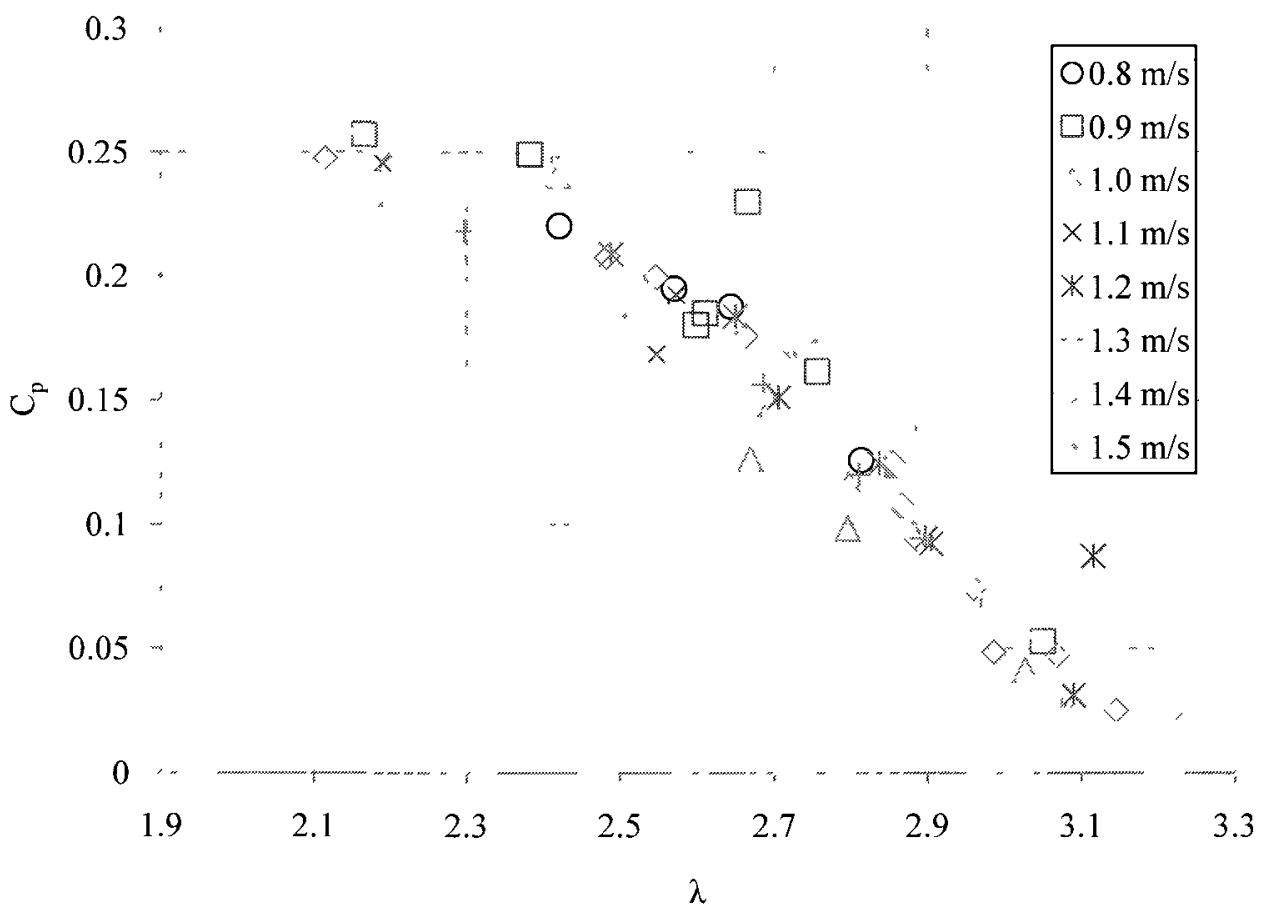

Figure 4.7 LST power coefficient versus tip speed ratio without blockage correction. Average error for $C_{p}=0.01$; for $\lambda=0.03$. 


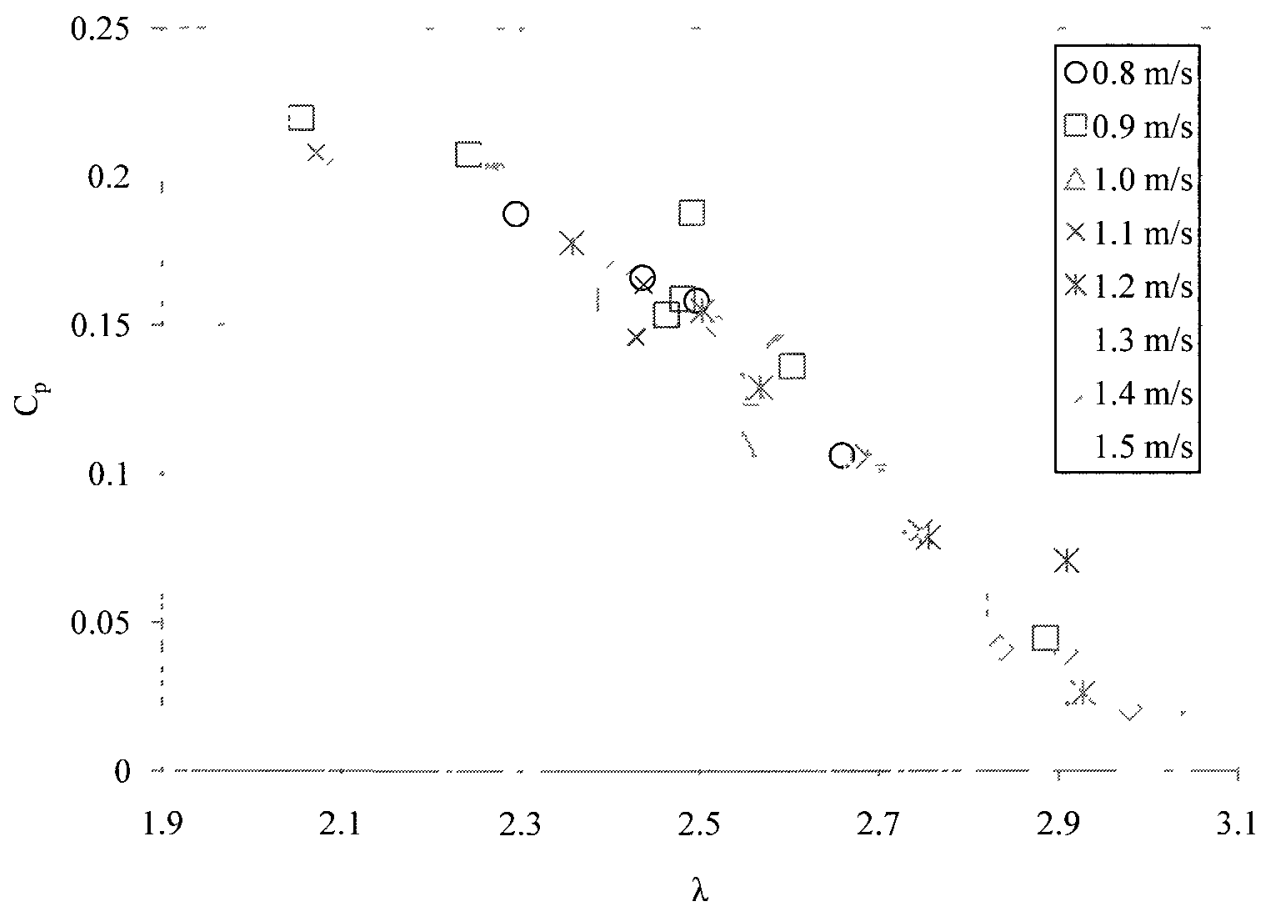

Figure 4.8 Blockage-corrected LST power coefficient versus blockage-corrected tip speed ratio.

Figure 4.9 and Figure 4.10 show the LST drag coefficient data plotted against tip speed ratio before and after blockage correction, respectively. Similar to the GHT, the values remain fairly constant with a slight trend toward higher $C_{\mathrm{d}}$ at higher $\lambda$. However, the LST overall has a lower drag coefficient. This can be partially attributed to its lack of center shaft. 


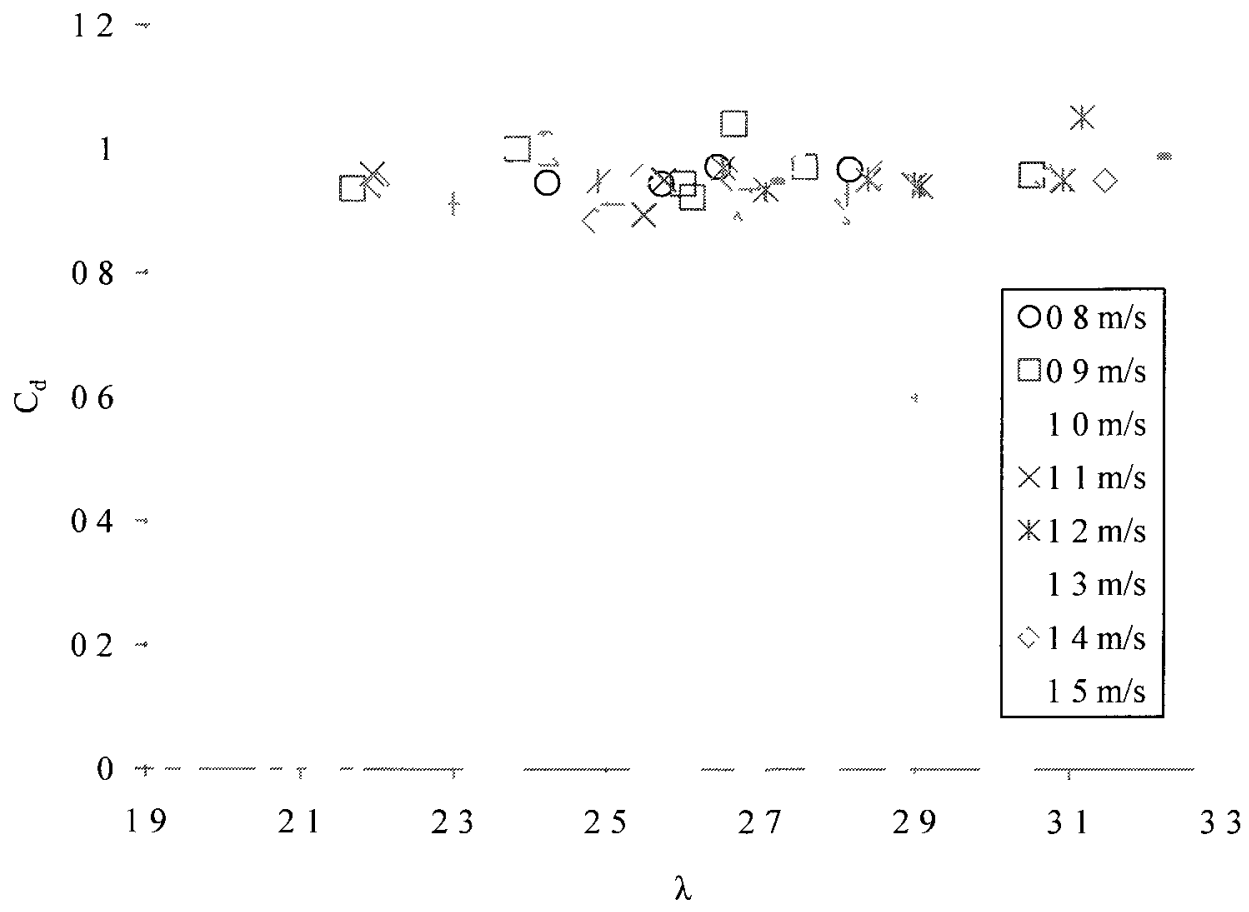

Figure 4.9 LST drag coefficient versus tip speed ratio without blockage correction. Average error for $C_{d}=0.002$; for $\lambda=0.03$.

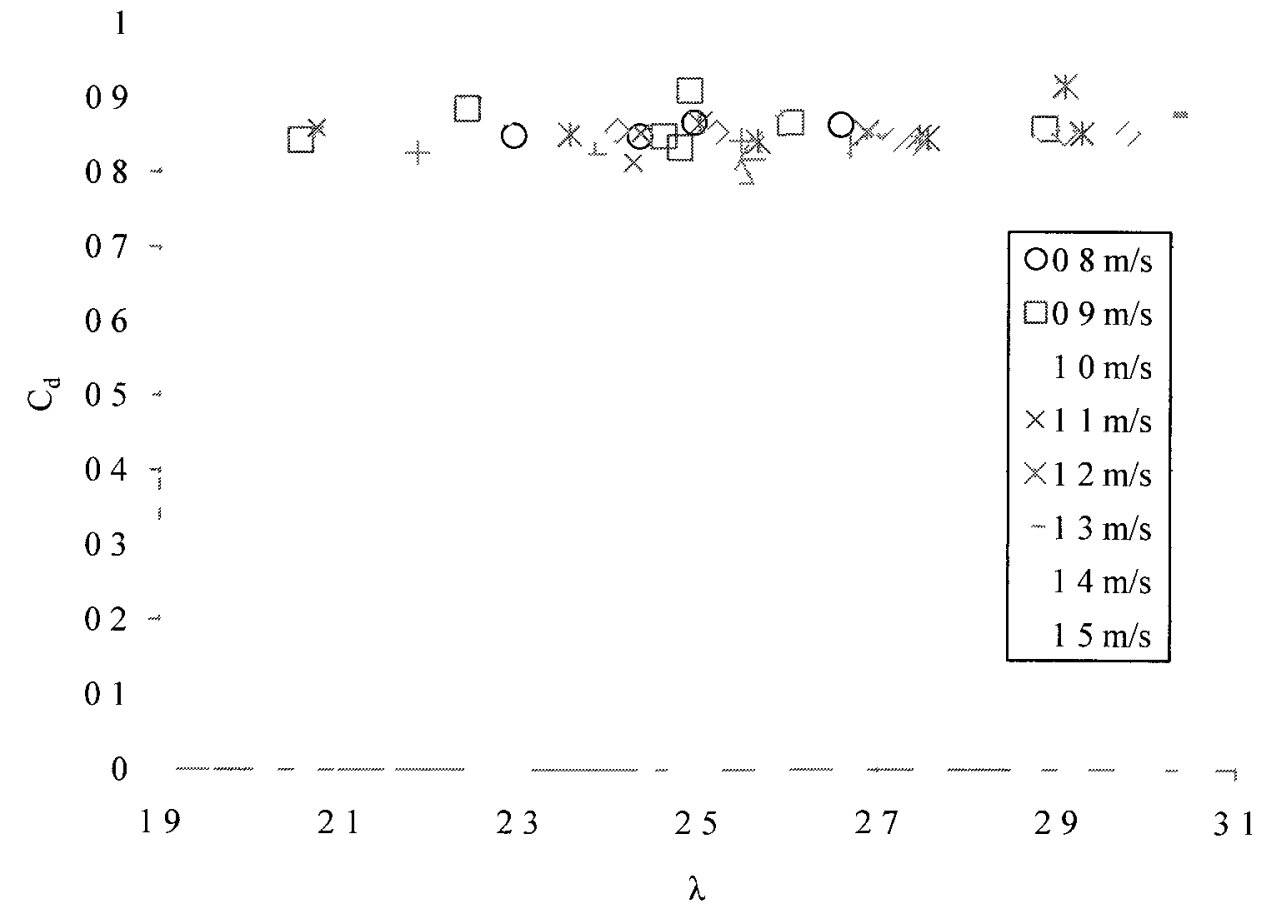

Figure 4.10 Blockage-corrected LST drag coefficient versus blockage-corrected tip speed ratio. 
Figure 4.11 shows LST kinetic exergy efficiency plotted versus tip speed ratio (calculated using power and drag coefficients that have not been corrected for blockage). Like the GHT, kinetic exergy efficiency plotted against tip speed ratio has the same general shape as the power coefficient versus tip speed ratio, and the maximum value around $90 \%$ corresponds to the tip speed ratio for maximum power coefficient.

The LST's lower radius at its poles could actually be helpful if inside a pipe boundary layer, where there will be lower fluid velocity, therefore lower angle of attack for a given tip speed ratio.

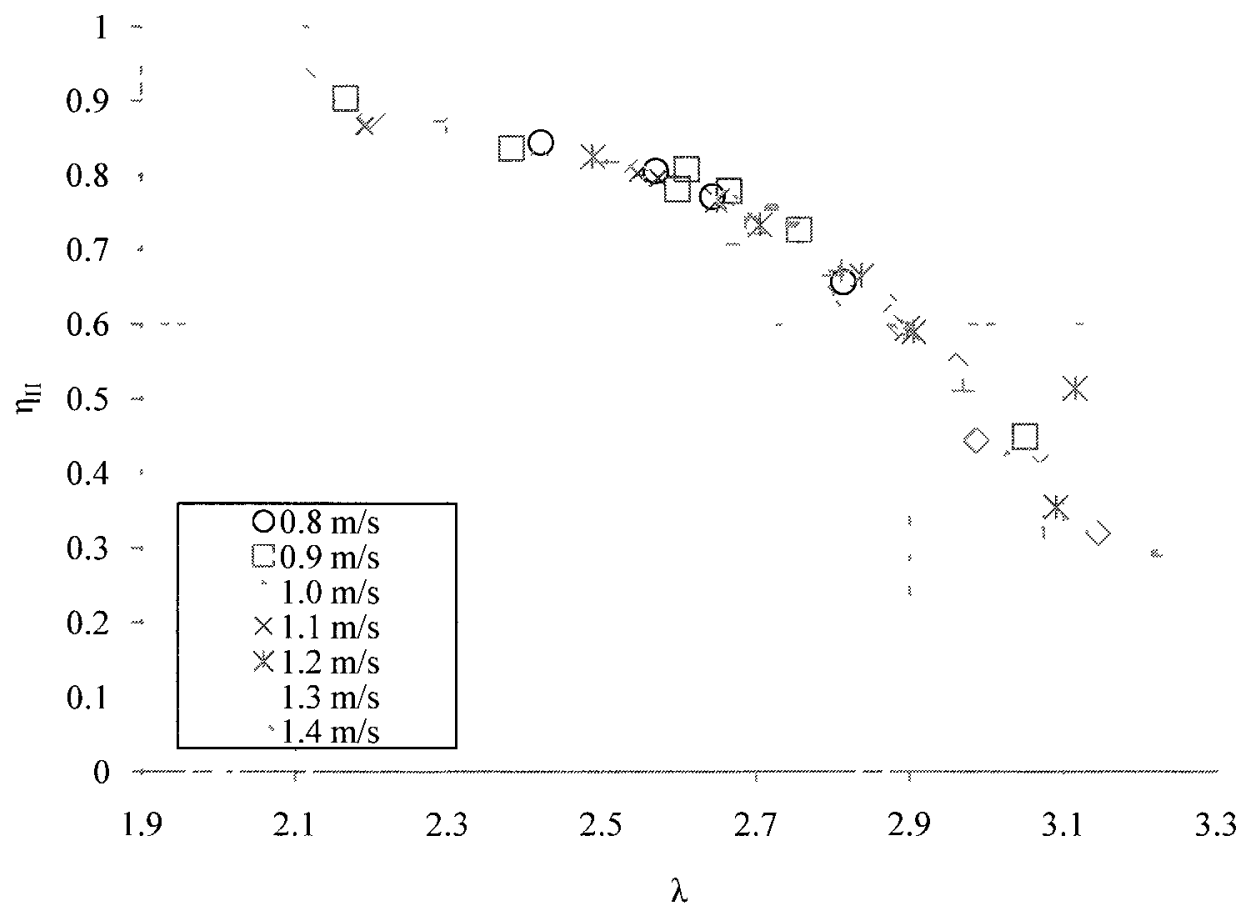

Figure 4.11 LST kinetic exergy efficiency versus tip speed ratio. 


\subsection{Tip Speed Ratio Limitations in Passive Loading Configuration}

Due to the nature of the experiments, during each run the turbine reaches its no load tip speed ratio and decelerates down to its operating point for a given load. In general, torque increases with decreasing tip speed ratio until blades begin to stall, which decreases hydrodynamic torque. The nondimensional torque coefficients, defined as

$$
C_{I}=\frac{T}{\frac{1}{2} \rho A_{f} U_{\infty}^{2}},
$$

is plotted versus tip speed ratio in Figure 4.12. This illustrates how hydrodynamic torque varies with tip speed ratio in the unstalled regime.

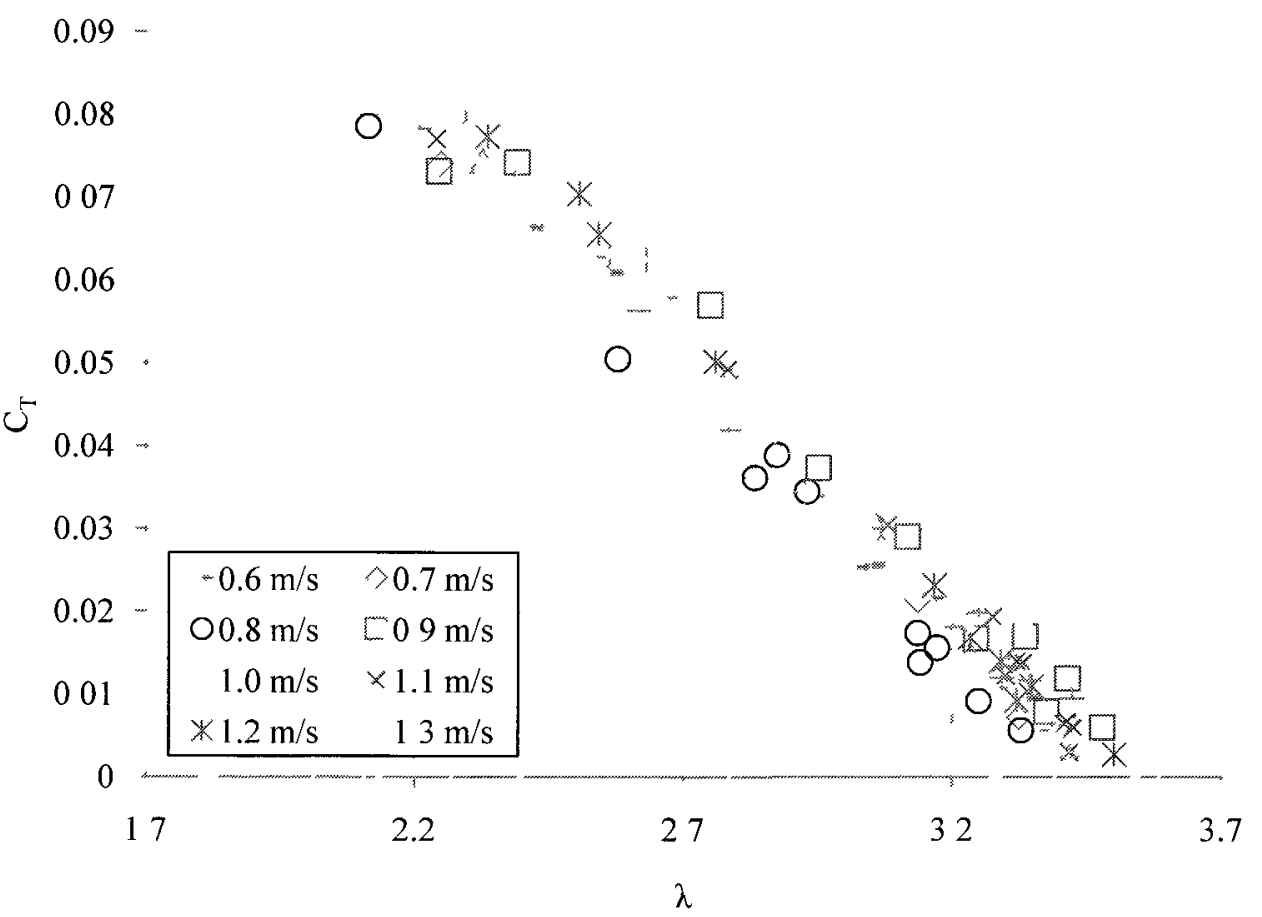

Figure 4.12 GHT torque coefficient versus tip speed ratio. 
Operating at tip speed ratios lower than those of peak torque will require some sort of control beyond a simple step loading. It may be possible to actuate the brake before it reaches high enough tip speed ratio to stay left of the torque peak, but that would possibly require a more complicated control and actuator system than an "active" style dynamometer, where the turbine tip speed ratio would be set by feedback controlled motor system, acting as a generator to produce load torque. Regarding the rigid body dynamics, if the turbine has a relatively low rotational inertia, the load (generator, controller, etc.) will need to have a fast frequency response for stable operation at tip speed ratios where stalling occurs. This effect has been seen in other experimental studies (McAdam, Houlsby, Oldfield, \& McCulloch, 2009).

\subsection{GHT Transient Measurements}

After sorting out the steady measurements, it was of interest to look at the dynamic measurements, i.e. cases where turbine RPM was not constant. In order to do this, the rotational inertia of the turbine and shaft was estimated to be $3 \mathrm{~kg} \cdot \mathrm{m}^{2}$ from the Solidworks CAD models. This rotational inertia was multiplied by the differentiated shaft angular velocity signal to estimate inertial torque. Figure 4.13 shows the transient data for one run at $U=1 \mathrm{~m} / \mathrm{s}$ versus the steady measurements. 


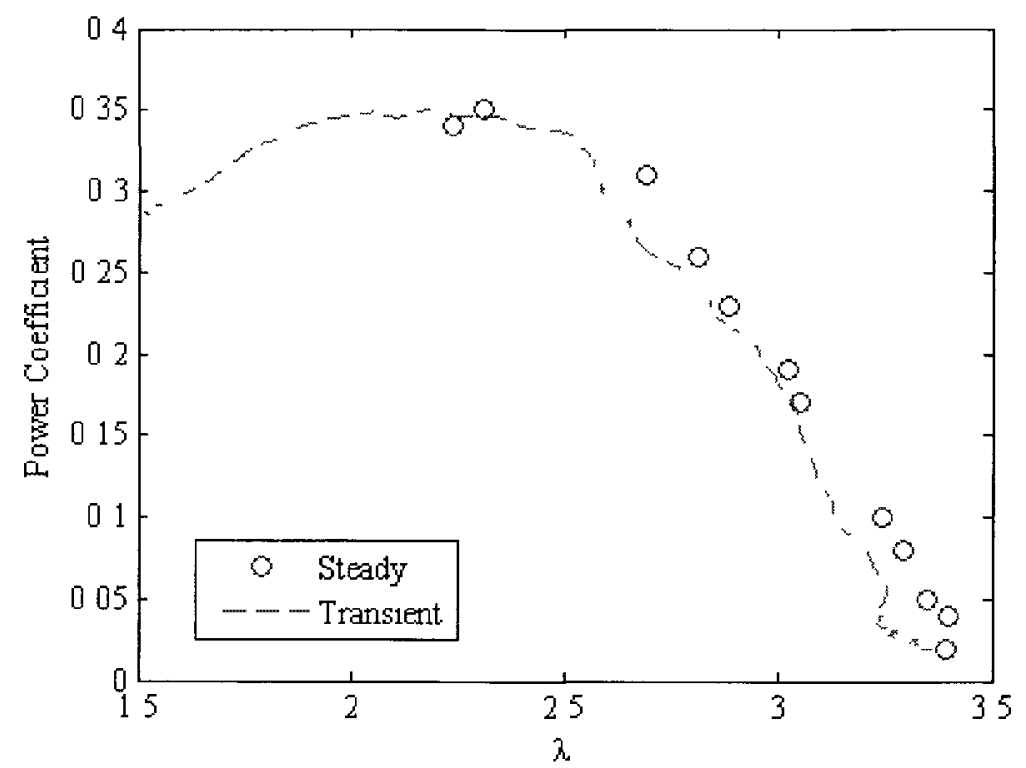

Figure 4.13 GHT steady and transient power coefficients versus tip speed ratio for $U=$ $1 \mathrm{~m} / \mathrm{s}$.

This shows it may be possible to get the entire operating envelope from one tow, as long as the rotational inertia can be measured accurately enough. This data also extends into the dynamic stall regime, though the flow during these transient measurements is likely different from that when shaft RPM is constant.

\subsection{Conclusions - Steady Performance}

Firstly, comparing the two devices, the GHT is a more effective converter of fluid kinetic energy than the LST. The GHT reached a maximum power coefficient of $36 \%$ whereas the LST only reached $25 \%$. This is to be expected given that the LST's intended environment is quite different from this low blockage tow tank scenario. The LST also tends to operate a lower tip speed ratios due to its higher solidity.

In the operating range of tip speed ratios seen here, drag coefficients remain quite constant, slightly increasing with tip speed ratio. The LST is in general a lower drag 
device compared to the GHT. Both devices have drag coefficients near unity, showing that they behave almost like solid bluff bodies when rotating. It would be interesting to see how the LST would perform with its center shaft installed; also, the GHT without its center shaft installed. These objects only provide resistance to flow through the turbine.

Regarding kinetic exergy efficiency, at peak power output, both devices convert roughly $90 \%$ of the streamwise kinetic energy they remove from the flow into shaft work. This shows that although the LST captures less of the fluid's available energy, it's not "wasting" any more energy than the GHT, so the energy is still available in the flow for other devices to capture. The remaining $10 \%$ not converted to shaft work could have been converted to heat via viscous dissipation or the mean kinetic energy could have been transformed to rotational or turbulent kinetic energy.

Transient power coefficient data seems to correspond well to the steady data with an estimate for rotational inertia. It should be investigated further as to whether this technique is a valid way to compare to, or even predict steady performance.

Note that there could be an introduction of error into the steady measurements from small unsteadiness inherent in the experiments. For example, change in carriage speed will cause inertial forces to alter drag measurements. Likewise, changes in shaft RPM will alter torque measurements. 


\section{Turbine Performance in Waves}

The presence of a progressive surface wave induces a periodic velocity fluctuation whose amplitude decreases with depth from the equilibrium surface. This can be derived analytically from linear (Airy) wave theory (Dean \& Dalrymple, 1991), as shown in Chapter 2.

Since a cross-flow axis turbine rotates in the same direction regardless of the direction of incoming flow, one might hypothesize these devices could extract energy even with a vertical axis in the plane of wave induced fluid velocity. For this reason, one would not expect an in-stream axis turbine to see any increase in power output due to progressive waves. One question that arises is whether there are limits of the frequency of flow reversal at which CFA devices can no longer extract power. For example, a tidal flow is an oscillating velocity in more or less one dimension over a relatively long period while a propagating surface wave period, therefore its period of fluid velocity oscillation is significantly shorter. Of interest is the upper limit of the ratio of turbine rotational period to velocity oscillation period at which the turbine can still convert fluid kinetic energy to shaft work.

Turbine performance for the GHT was measured while being towed $1.0-1.1 \mathrm{~m} / \mathrm{s}$ against the direction of propagation of four different types of $0.15 \mathrm{~m}$ high waves, having periods $1,1.5,2$, and $2.5 \mathrm{~s}$. Figure 5.1 is a sketch illustrating the approximate relative size of particle orbits, tank depth, wave height, and turbine dimensions. Figure 5.2 shows how the amplitude of the theoretical horizontal wave velocity and particle displacement vary 
with depth, as calculated from Equation (2.38). These parameters averaged over turbine height are shown along with theoretical wavelength in Table 5.1.
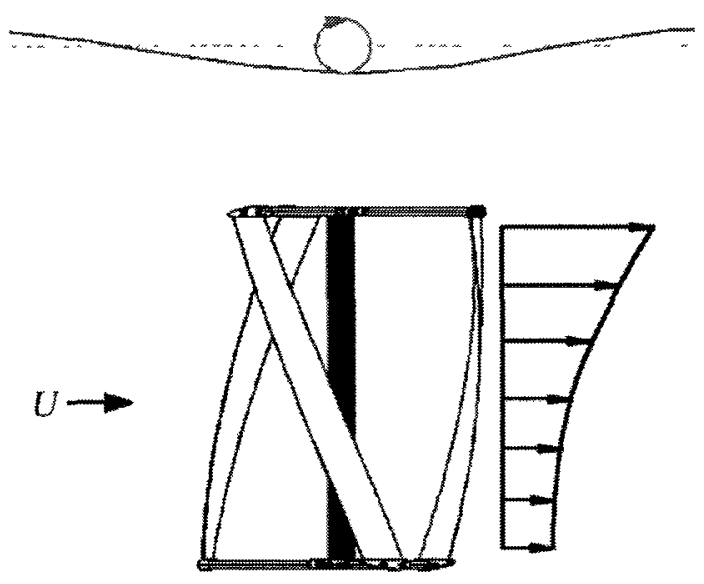

Figure 5.1 Sketch of the GHT operating under a wave in the tow tank.

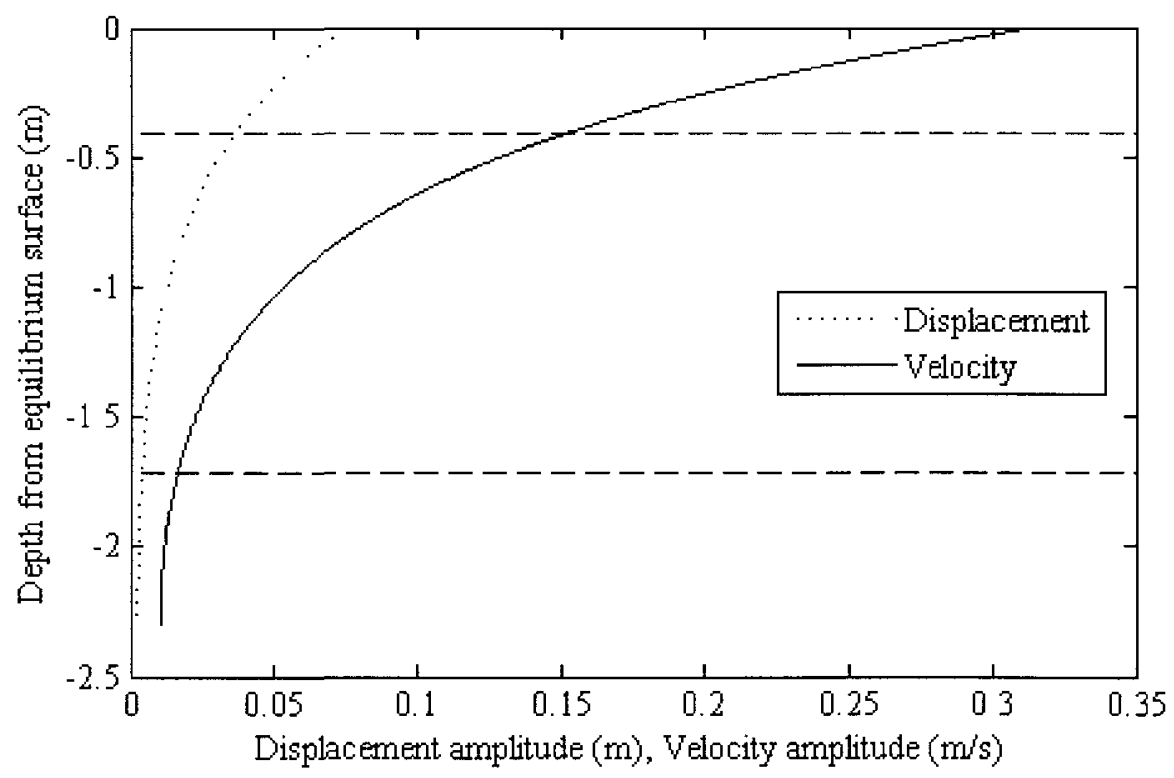

Figure 5.2 Theoretical horizontal wave velocity and displacement amplitudes versus depth for a $0.15 \mathrm{~m}$ high $1.5 \mathrm{~s}$ period wave. Dashed horizontal lines indicate turbine location. 
Table 5.1 Theoretical wave parameters averaged over turbine height.

\begin{tabular}{ccccc}
\hline Period $(\mathrm{s})$ & 1.0 & 1.5 & 2.0 & 2.5 \\
\hline Height $(\mathrm{m})$ & 0.15 & 0.15 & 0.15 & 0.15 \\
Wavelength $(\mathrm{m})$ & 1.6 & 3.5 & 6.1 & 9.0 \\
Horiz. disp. amp. $(\mathrm{m})$ & 0.003 & 0.01 & 0.03 & 0.05 \\
Horiz. vel. amp. $(\mathrm{m} / \mathrm{s})$ & 0.02 & 0.06 & 0.09 & 0.1
\end{tabular}

\subsection{Results - GHT Performance in Waves}

Figure 5.3 shows a sample data collection run, including ADV measurements in the streamwise, cross-stream, and vertical directions for a carriage speed of $1 \mathrm{~m} / \mathrm{s}$ towed in a $2.5 \mathrm{~s}$ period progressive wave. Notice how in the sample run the carriage velocity fluctuates from the fluctuating drag on the turbine caused by the waves. This extra carriage motion could be adding or subtracting to the streamwise component of wave induced velocity, which needs to be kept in mind when observing the results, though smaller wave periods had less effect. At the very least, this observation shows the need for more carriage position damping, a stiffer tow mechanism, and closed loop speed control for the tow system. Note how the horizontal and vertical wave-induced velocities are captured by the ADV measurements, and how the cross-stream velocity is non-zero, showing how flow tends to "avoid" the turbine. 


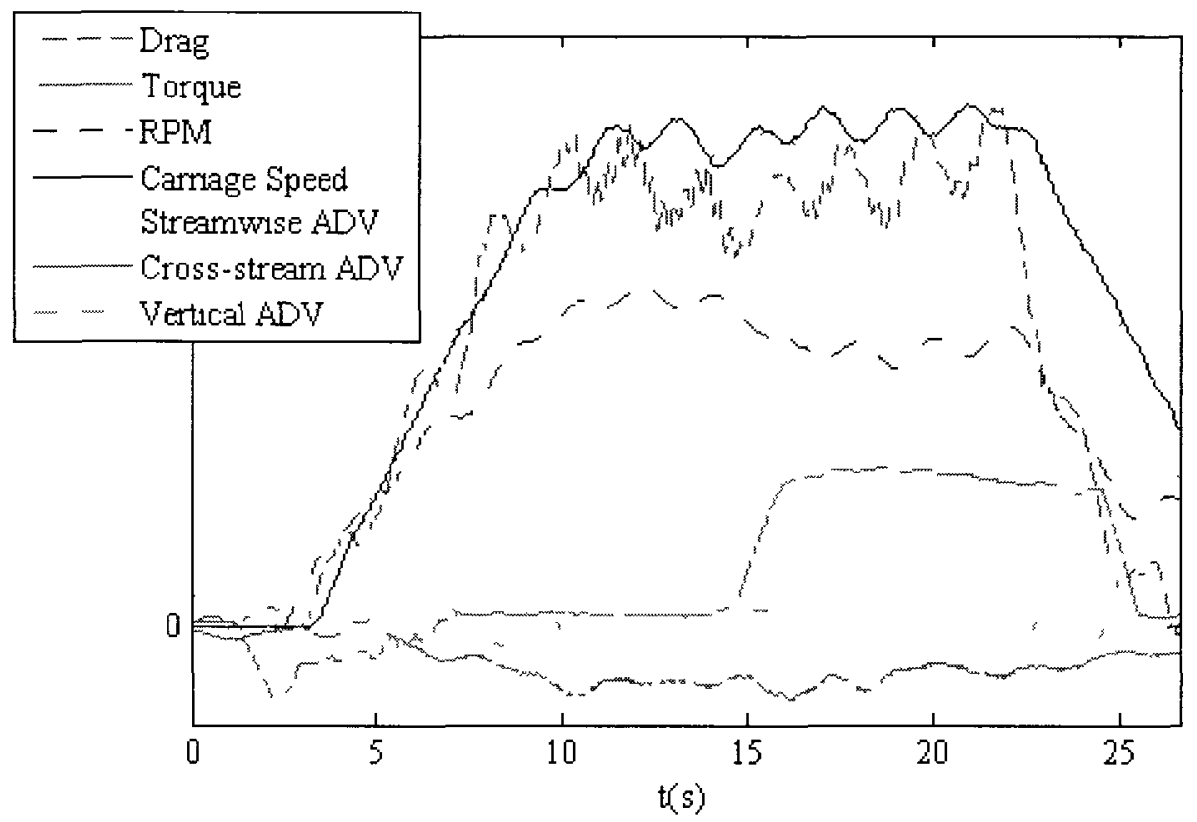

Figure 5.3 Sample data collection run in $2.5 \mathrm{~s}$ period waves (in arbitrary units).

Figure 5.4 shows GHT power coefficient plotted versus tip speed ratio (not corrected for blockage) for carriage speeds of $1.0-1.1 \mathrm{~m} / \mathrm{s}$. In general, an increase in power coefficient and tip speed ratio is observed towing in waves, including an approximate $11 \%$ increase in maximum power coefficient in the $1.5 \mathrm{~s}$ period wave. With increasing brake torque, the turbine stalled at higher tip speed ratios in waves than in steady flow. For the longest wave period, $2.5 \mathrm{~s}$, the turbine stalled at a much higher tip speed ratio than usual. An attempt to explain this behavior is made in the following section. Drag coefficients, plotted in Figure 5.5, are also higher than the steady flow case. 


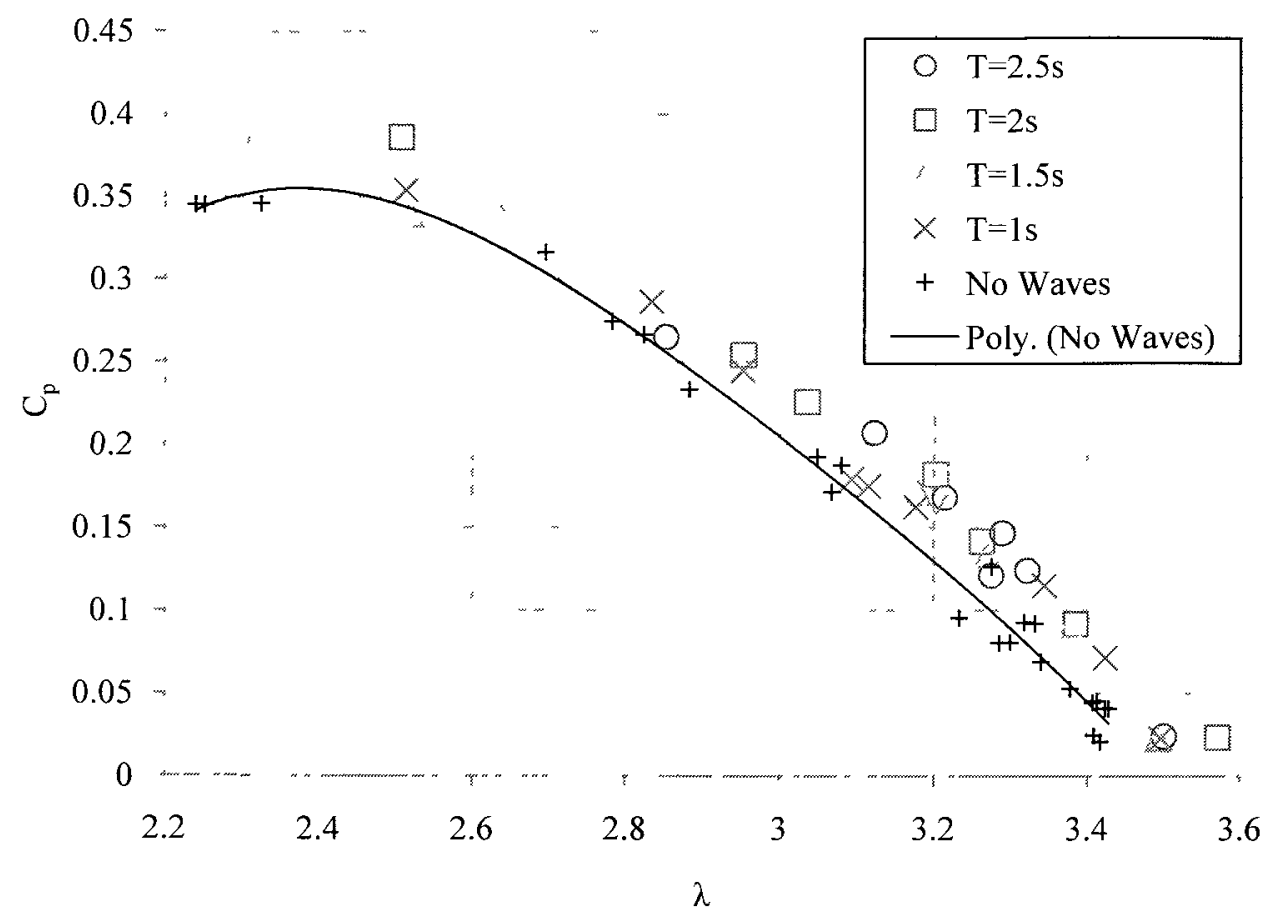

Figure 5.4 GHT power coefficient versus tip speed ratio for $U=1.0-1.1 \mathrm{~m} / \mathrm{s}$ with and without $0.15 \mathrm{~m}$ high waves present.

1.4

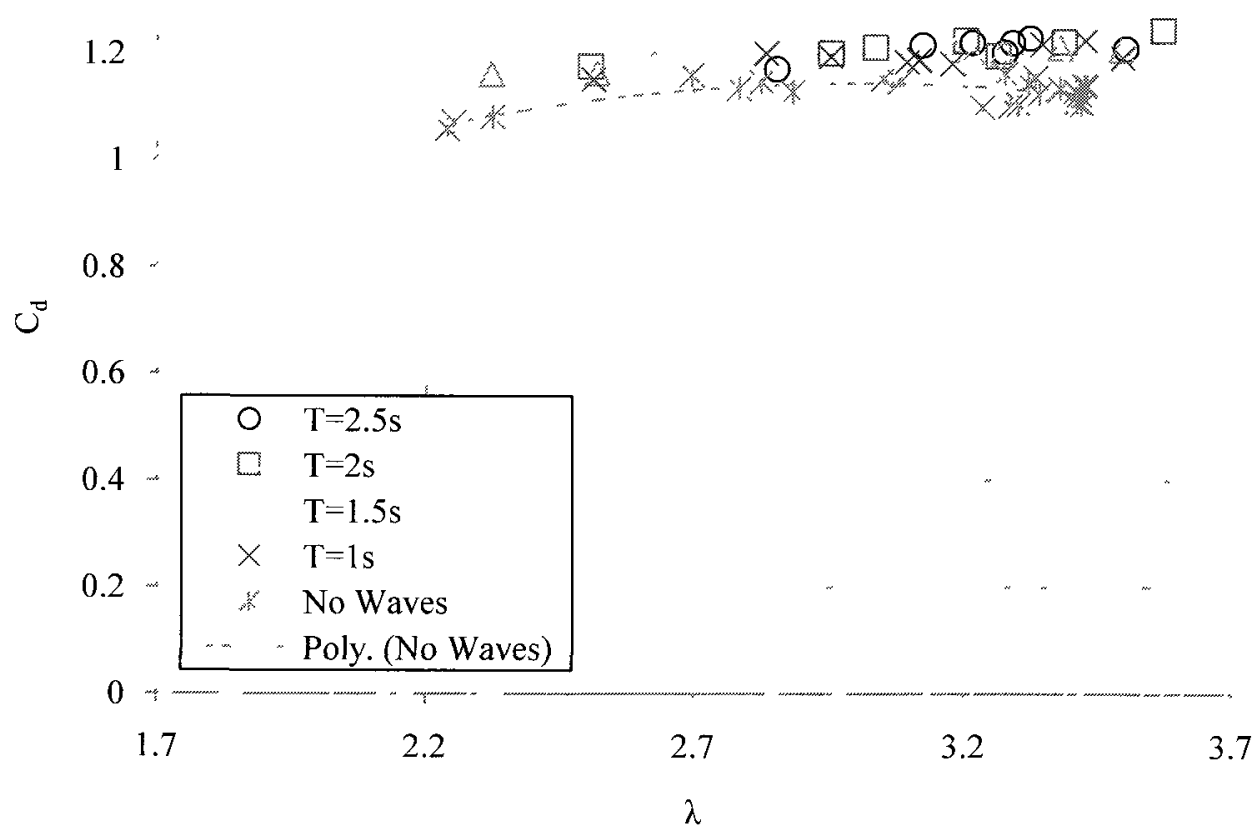

Figure 5.5 GHT drag coefficient versus tip speed ratio for $U=1.0-1.1 \mathrm{~m} / \mathrm{s}$ with and without $0.15 \mathrm{~m}$ high waves present. 


\subsection{Developing a 2D Model to Explain Results}

To attempt to explain some of the observed effects of waves, a 2D blade element type method was developed using three equally spaced $14.8 \mathrm{~cm}$ chord blades at radius $r=0.5$ m (similar to the GHT), using static lift and drag coefficient data for a NACA 0020 taken from (Johnston, 2011). This dataset was created by linearly interpolating between the NACA 0018 and NACA 0021 datasets in (Sheldahl \& Klimas, 1981). Using the equations presented in Chapter 2, angle of attack and relative velocity are calculated. Reynolds number is calculated with respect to relative velocity and chord length. Using angle of attack and Reynolds number, the lift and drag coefficients are taken from the interpolated dataset, from which lift and drag are calculated. Streamwise induction factor is estimated by first setting induction as a constant value (uniform across the entire turbine), running the model, and estimating at which tip speed ratio the power coefficient intersects the experimental data. Repeating this process for a range of induction factors produces an approximate relationship for how induction varies with tip speed ratio. Power coefficient curves for constant induction are shown in Figure 5.6. 


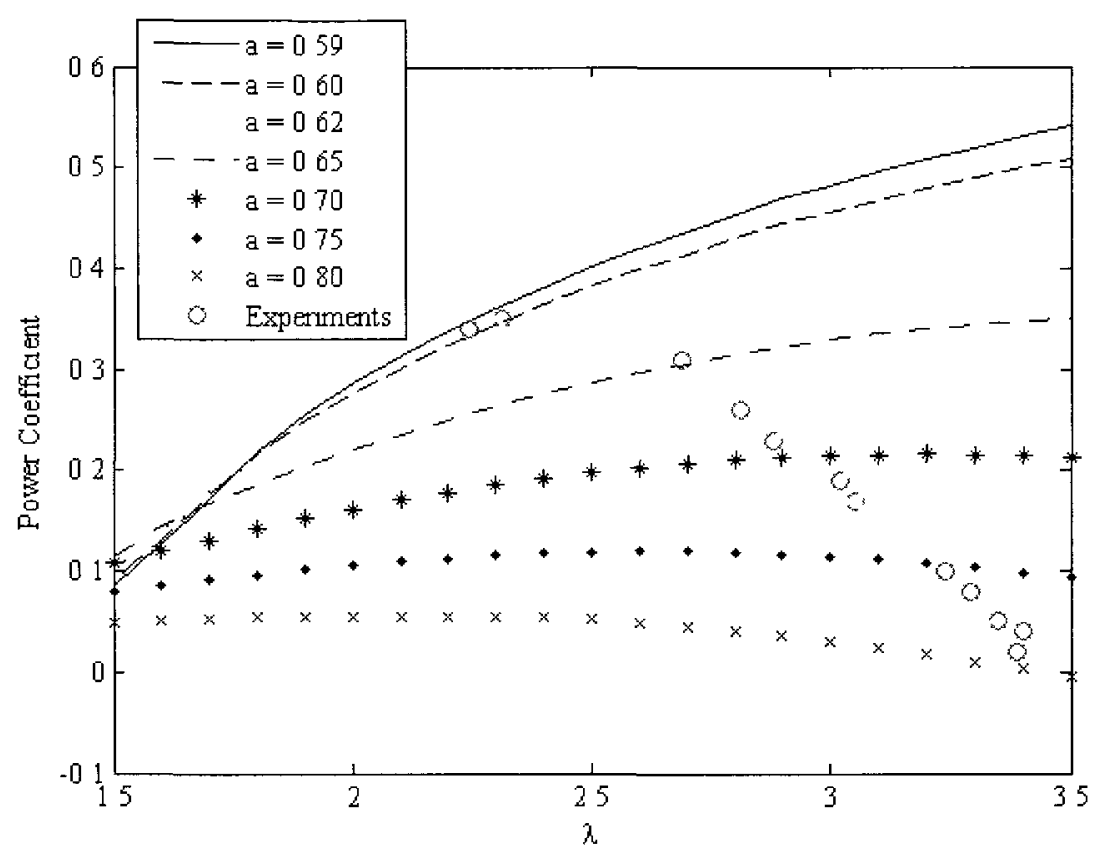

Figure 5.6 Model power coefficient for various constant induction factors and experimental results plotted versus tip speed ratio.

A linear regression between streamwise induction and tip speed ratio, $a=0.1838 \lambda+$ 0.1623 , is chosen to fit the data the best. The positive slope of this relationship makes sense since the turbine should slow down the incoming flow more as it becomes more solid "in time" with increasing tip speed ratio. Another way to look at this is the likelihood of a fluid particle transferring its momentum to a blade increases when the blades are moving faster compared to the free stream.

Once the induction relationship is built into the model, turbine power and drag coefficients are plotted versus tip speed ratio for the steady case, in Figure 5.7 and Figure 5.8 , respectively. Also plotted are the results from the $1 \mathrm{D}$ momentum equations in Chapter 2, using the estimated streamwise induction. The close match of the model with experimental data for both power and drag shows the validity of this induction approximation, but there are no flow data to confirm. It's remarkable how well the drag 
coefficient is predicted at tip speed ratios below 3 in spite of the model being "calibrated" with only power coefficient data. The induction estimate used also makes sense when looking at the amplitude of the theoretical angle of attack at which the turbine stalls in experiments, which approximately corresponds to the foil's static stall angle. This also shows that for the experiments, the blades may only reach static stall when the turbine stops, so dynamic stall may not be encountered.

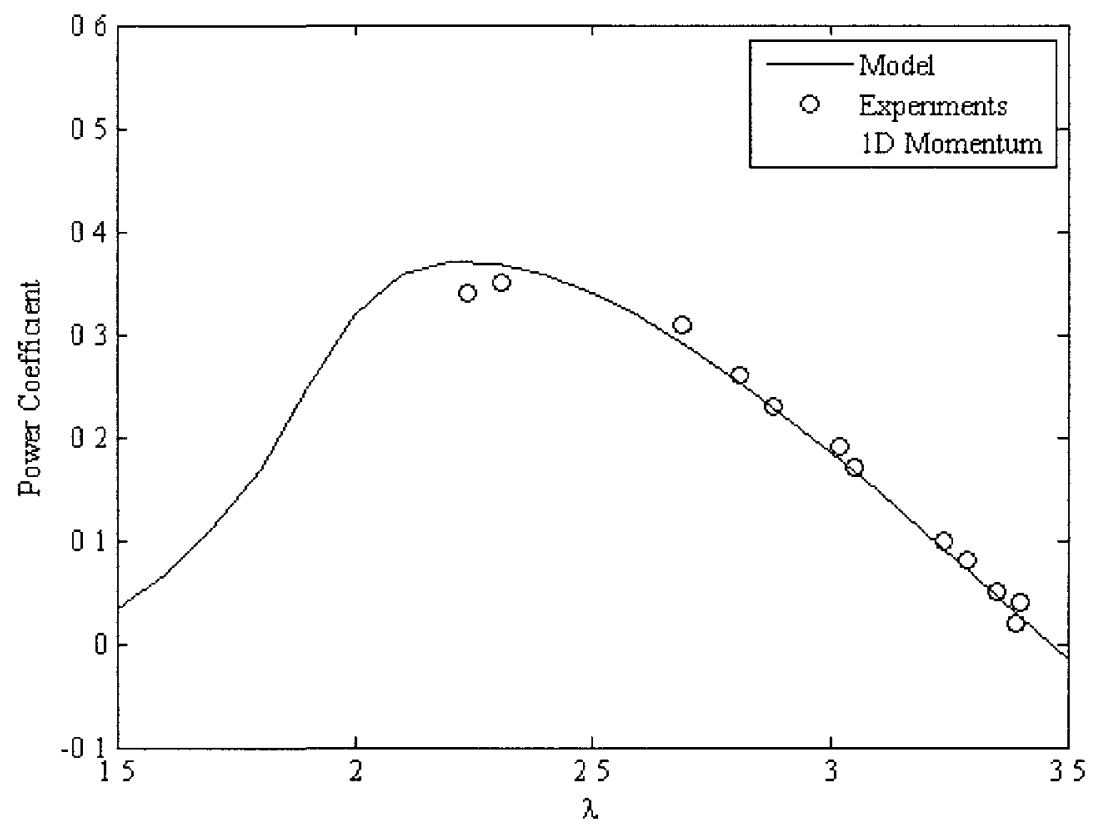

Figure 5.7 Power coefficient versus tip speed ratio for steady model at $U=1 \mathrm{~m} / \mathrm{s}$, experiments at $U=1 \mathrm{~m} / \mathrm{s}$, and results from $1 \mathrm{D}$ momentum theory based on induction estimates. 


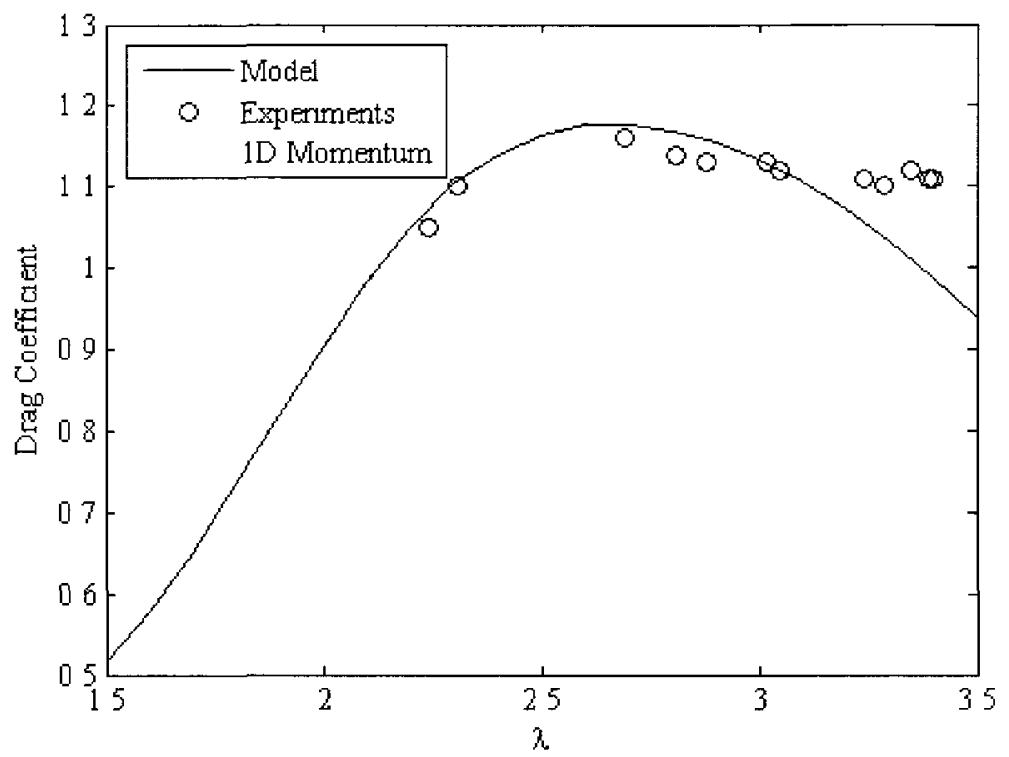

Figure 5.8 Drag coefficient versus tip speed ratio for steady model at $U=1 \mathrm{~m} / \mathrm{s}$, experiments at $U=1 \mathrm{~m} / \mathrm{s}$, and results from $1 \mathrm{D}$ momentum theory.

This model can be used to explain the lower bound of tip speed ratios encountered in the experiments. Figure 5.9 shows the theoretical lift to drag ratio versus angle of attack calculated with the model for tip speed ratios of 2.0 and 2.3 , the latter being near the tip speed ratio corresponding to peak torque and the former being lower. For a tip speed ratio of 2.3 it can be seen that the blade comes close to stall while at a tip speed ratio of 2.0 the blade certainly enters stall, explaining the drop in average torque at the shaft. 


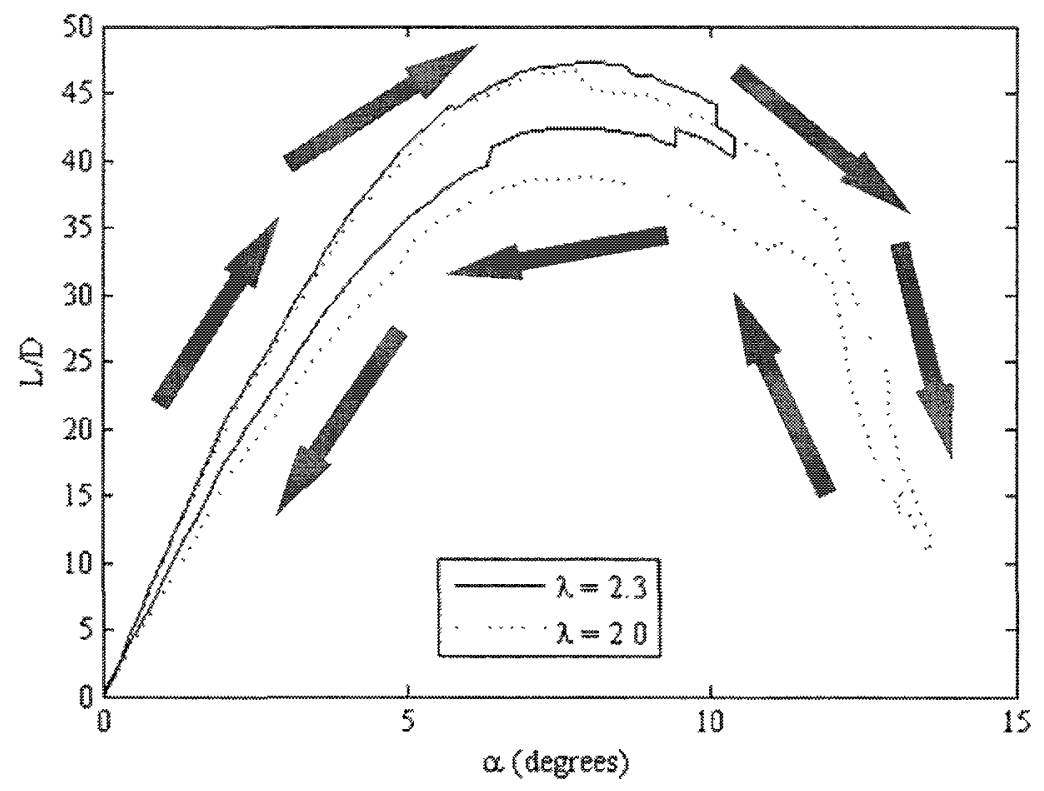

Figure 5.9 Lift to drag ratio versus angle of attack for one half rotation, for two tip speed ratios at $U=1.0 \mathrm{~m} / \mathrm{s}$, calculated with the $2 \mathrm{D}$ model. Arrows show progression of turbine rotation angle or time, with the origin corresponding to $\theta=0^{\circ}$ or $180^{\circ}$.

Figure 5.10 shows the spectrum of the theoretical torque contributions of all three blades. Beyond a tip speed ratio of 2.2 or so, there is very little variance, or "torque ripple," compared to when tip speed ratio is below 2.2 This theoretical variance is mainly due to blade stall, hence it occurs at three (the number of blades) times the turbine rotational frequency and should be taken into account when specifying the required frequency response of a turbine loading system if it will encounter stall, for example from sudden increases in free stream velocity. 


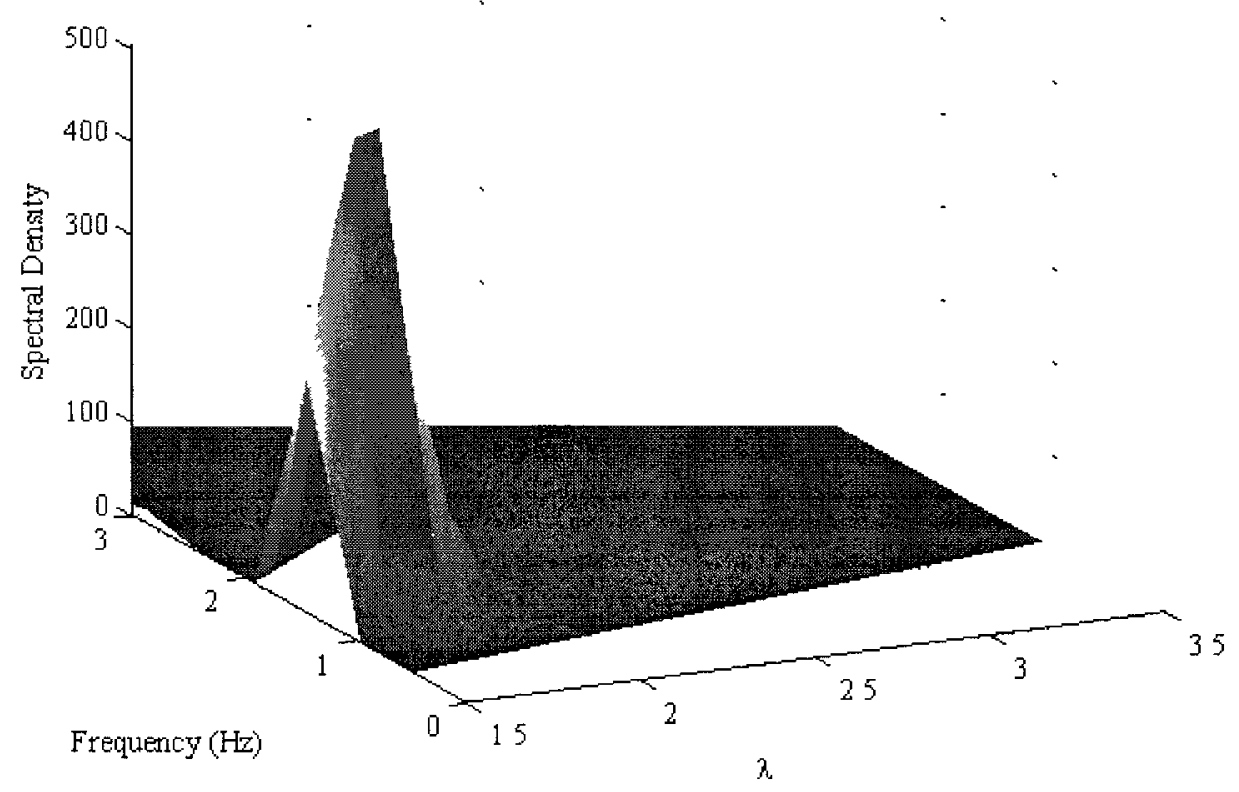

Figure 5.10 Spectrum of model output torque for various tip speed ratios without waves present.

The 2D model was then modified to attempt to analyze the effects of the waves' streamwise velocity fluctuations on turbine performance. First, wavenumber, $k$, is solved for iteratively with the wave and tank parameters using the linear dispersion relation (Dean \& Dalrymple, 1991),

$$
\sigma^{2}=g k \tanh (k h),
$$

to an accuracy of

$$
g k \tanh (k h)-\sigma^{2}<0.001,
$$

where $\sigma$ is the wave radian frequency. Next, the amplitude of velocity fluctuation is calculated using Equation (2.38), and is averaged over the turbine's height in the tank. This fluctuating velocity is then added to the free stream multiplied by $(1-a)$, the 
estimated effect of streamwise induction. The incident wave velocity at a blade takes into account the three different blade positions changing with rotation angle and time as

$$
x_{\text {blade }}=r \sin \theta_{\text {blade }}-U t .
$$

Figure 5.11 shows the model's calculated power coefficient plotted versus tip speed ratio averaged over six rotations under a $1.5 \mathrm{~s}$ wave along with steady experimental results at $U=1 \mathrm{~m} / \mathrm{s}$.

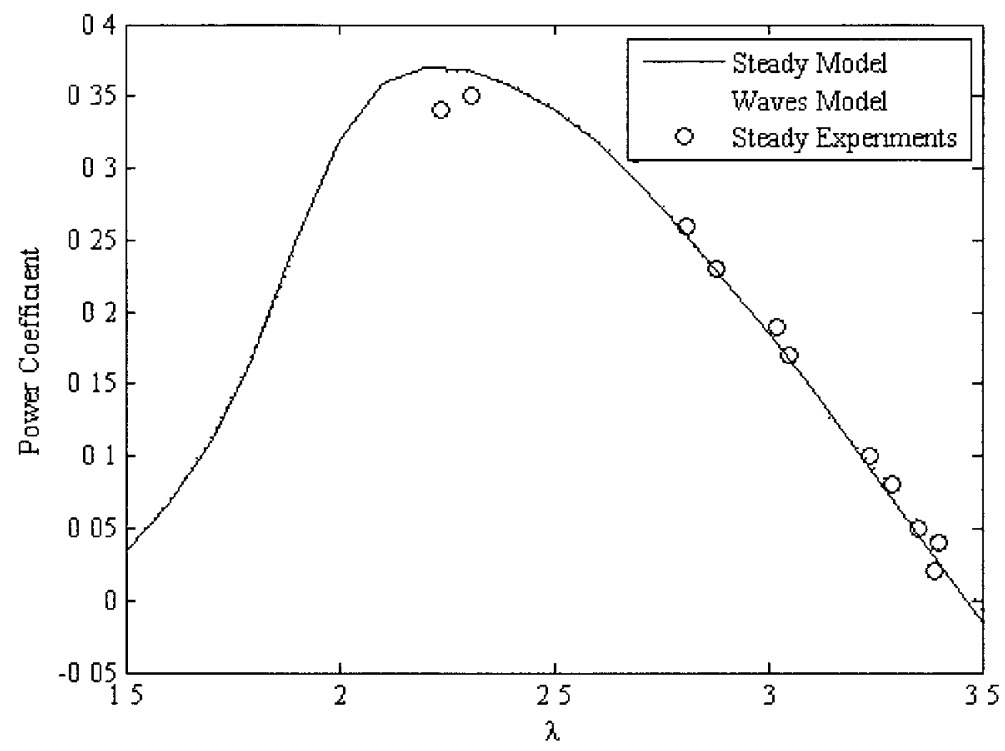

Figure 5.11 Power coefficient versus tip speed ratio calculated for six rotations with and without $1.5 \mathrm{~s}$ period waves present in the $2 \mathrm{D}$ model at $U=1 \mathrm{~m} / \mathrm{s}$.

Looking at Figure 5.11, something is apparently not accurate in the model since experiments showed substantial increases in power coefficient, shown in Figure 5.4. Perhaps the lack of three dimensionality in the model, failing to account for blade sweep, variation of wave velocities with height, etc., are causing this discrepancy. Despite the lack of the ability to predict power increases, the model can show how an increase in torque variance may be caused by the presence of a wave, causing unstable operation at 
lower tip speed ratios. This variance is due to the wave velocity pushing angle of attack beyond the stall point for different blades at different times.

Figure 5.12 shows the model torque variance predicted under a $2.5 \mathrm{~s}$ period wave. Looking back at Figure 5.4, the lowest tip speed ratio at which the turbine would operate in experiments was 2.85. Figure 5.12 shows that below this tip speed ratio there is a large increase in low frequency variance of the torque output. If this were present during the experiments, the turbine would decelerate during times of lower torque, lowering tip speed ratio, worsening blade stall, and would eventually lead to the halting of the turbine altogether. This could possibly explain the experimental observations.

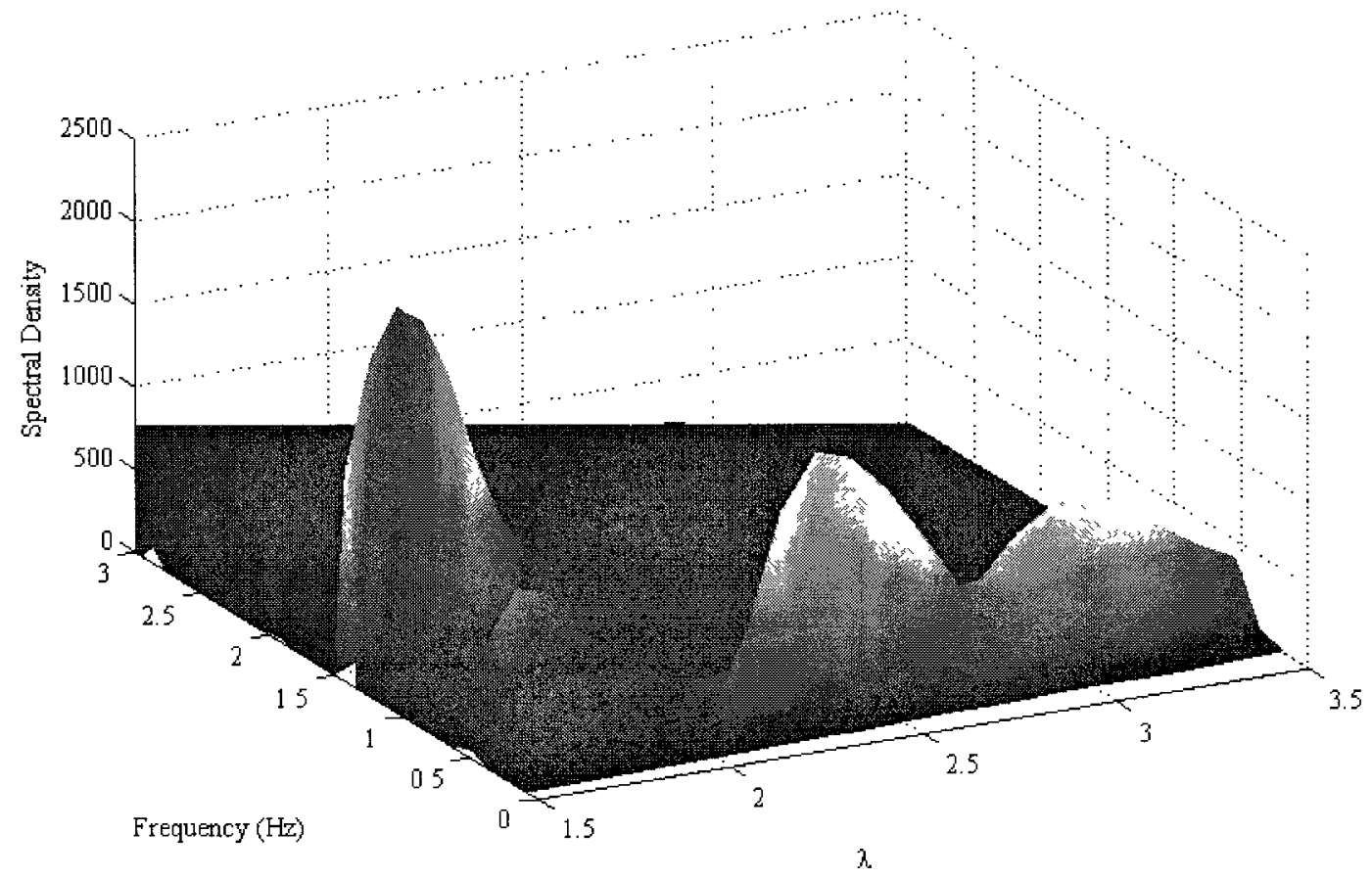

Figure 5.12 Torque spectral density predicted by the model under a $2.5 \mathrm{~s}$ period wave. 


\subsection{Conclusions - Performance in Waves}

GHT power coefficients in waves are higher than those in steady flow. A simplified 2D blade element type model developed cannot adequately explain why the turbine power output is increased. However, the model does show that the presence of waves is a source of low frequency variance in the torque output, therefore explaining the experimental observations that stable operation was not possible with the passive dynamometer setup below certain tip speed ratios. The additional low frequency components of the torque signal shown by the model have implications for turbine and generator design, namely in the inertia of the rotor and the frequency response of any generator system. At the very least, for potential deployments, wave induced velocities should be taken into account for modeling and control purposes.

In the future it is necessary to observe how the turbine operates in wave conditions more akin to what would be present in real world tidal flows. It is also necessary to perform more experiments with a feedback controller for tip speed ratio, to observe whether or not power output will still increase under the longer period waves at lower tip speed ratios. Another question is whether or not the direction of propagation will affect the observations. 


\section{Turbine Performance in Turbulence}

Since a real world flow in which these turbines might be placed is surely turbulent, it was of interest to observe changes in performance due to introduction of turbulence to the inflow. For example, a tidal flow has an approximate Reynolds number of 1 million per $\mathrm{m}$ depth and per $\mathrm{m} / \mathrm{s}$ flow velocity. The blade element Reynolds number for a cross-flow (vertical or horizontal) axis turbine can be written as

$$
\operatorname{Re}_{c}=\left(\rho U_{\infty} c\left(1+2 \lambda(r / R) \cos \theta+\lambda^{2}(r / R)^{2}\right)^{1 / 2} / \mu\right),
$$

while for an in-stream (horizontal) axis turbine it can be written as

$$
\operatorname{Re}_{c}=\left(\rho U_{\infty} c\left(1+\lambda^{2}(r / R)^{2}\right)^{1 / 2} / \mu\right),
$$

neglecting effects of induction for this argument. Here $c$ is the blade chord, $U_{\infty}$ is the free-stream velocity, $\lambda$ is the rotor tip speed ratio, $\mu$ is the dynamic viscosity, $\theta$ is the angle of rotation for the cross-flow axis turbine, and $r / R$ is the non-dimensional radial position. The US Department of Energy (DOE) has designed Marine Hydrokinetic (MHK) "reference turbines," to have fully specified turbines in the open literature for numerical and physical model studies. For the DOE MHK Reference Tidal Turbines (ORNL, Sandia NL, NREL, 2011), the blade Reynolds numbers vary between approximately 1 and 2 million for the $6.45 \mathrm{~m}$ diameter vertical axis reference turbine, and between 4 and 8 million for the $20 \mathrm{~m}$ diameter horizontal axis reference turbine for the given operating regimes. Hence, for these turbines a high Reynolds number turbulent flow interacts with high Reynolds number flow surfaces. For similar turbines that are smaller than the DOE reference turbines, the tip speed ratio in the scaled turbine is matched to achieve kinematic similarity of velocity vector diagrams, and effects due to a 
lower Reynolds number can come into play. However, the scale of the turbines used in this study is still sufficiently large that effects of turbulence will be important. This chapter presents first results of effects of turbulence generators on CFA hydrokinetic turbines. Data of this type do not yet exist in the literature.

In 2001, Swalwell et. al. measured lift and drag coefficients for a NACA 0021 foil in various grid turbulence intensities in a wind tunnel. Some of the results are reproduced in Figure 6.1. A delay of static stall to higher angles of attack was observed with increased turbulence intensity. Due to the delay of stall, lower drag coefficients were observed over a range of angles of attack from about 10 to 40 degrees as well. Turbulence enhances momentum transfer from the fluid to the wall of the hydrofoil, providing higher local momentum to help move fluid downstream against the adverse pressure gradient on the suction side of the foil. Turbulence thus increases friction drag (Reynolds stresses act like effective viscous stresses to transfer momentum), but reduces the pressure drag since the flow remains attached longer. If the pressure drag reduction is larger than the viscous drag increase the overall drag will decrease, which was observed here, c.f. Figure 6.1. 

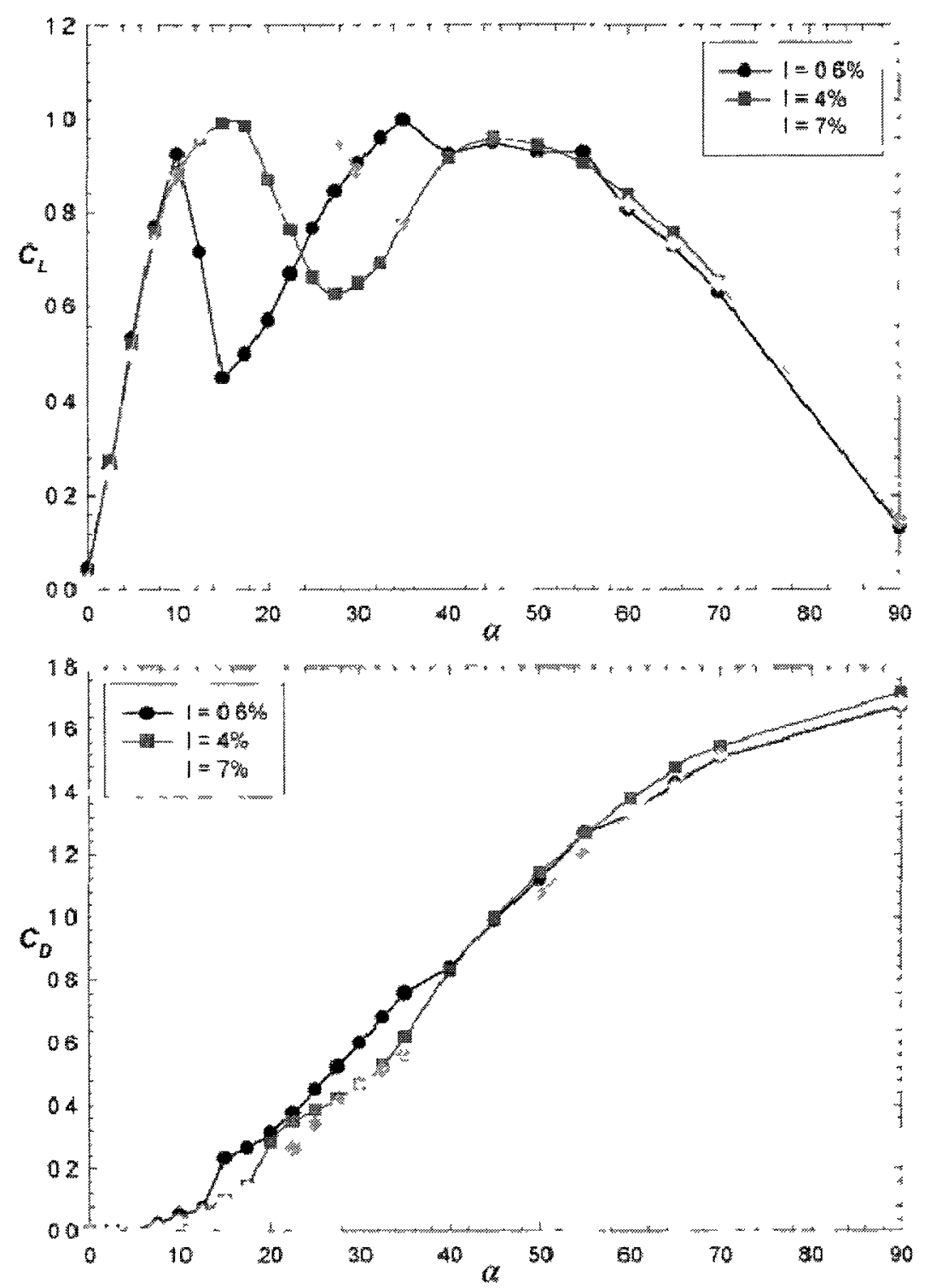

Figure 6.1 Lift (top) and drag (bottom) coefficients versus angle of attack for a NACA 0021 foil in various levels of grid turbulence. Taken from Swalwell, Sheridan, \& Melbourne (2001).

\subsection{Delay of Static Stall - PIV Measurements}

To observe the flow associated with the static stall delay phenomenon, a $36 \%$ solid grid with $3.18 \mathrm{~cm}$ mesh size was placed 10 mesh widths upstream of a $7 \mathrm{~cm}$ chord NACA 0012 hydrofoil in a small $15.2 \mathrm{~cm}(6$ inch) square cross section UNH water channel. The foil was placed at 9 degrees angle of attack in a $0.3 \mathrm{~m} / \mathrm{s}$ free stream velocity $\left(R e_{c}=\right.$ 
$21,000)$. Figure 6.2 shows lift over drag ratio data for a NACA 0012 at higher Reynolds numbers, showing how stall angles increase with increasing Reynolds number. Flow was measured using high frame-rate stereo PIV. A photograph of the experimental setup is shown in Figure 6.3.

The Field of View (FOV) was $120 \mathrm{~mm} \times 120 \mathrm{~mm}$, for a magnification factor of 0.145 (CMOS sensor dimensions are $17.408 \mathrm{~mm}$ x $17.408 \mathrm{~mm}$ ). 4000 PIV images were acquired at $400 \mathrm{~Hz}$, producing 10 seconds worth of data. The raw data were processed using an FFT cross-correlation with a final interrogation spot size of $16 \times 16$ pixels with $50 \%$ overlap, resulting in a "structural resolution" of $1.875 \mathrm{~mm}$.

Results for the hydrofoil's instantaneous velocity and vorticity fields with streamwise turbulence levels of $1 \%$ (tunnel background turbulence) and $5 \%$ (with upstream grid), defined as (Panton, 1933)

$$
I_{\imath}=\frac{R M S\left(u_{i}^{\prime}\right)}{U_{\infty}},
$$

are shown in Figure 6.4. Figure 6.5 shows mean velocity magnitude contours for each case normalized by its respective free stream velocity averaged across a rectangular region with corners at $(40,-26)$ and $(50,-19) \mathrm{mm}$. In the higher turbulence case, the wake appears to be thinner (though the effect is subtle), indicating a reduction in pressure on the lower surface, hence higher lift compared with the lower turbulence case. 


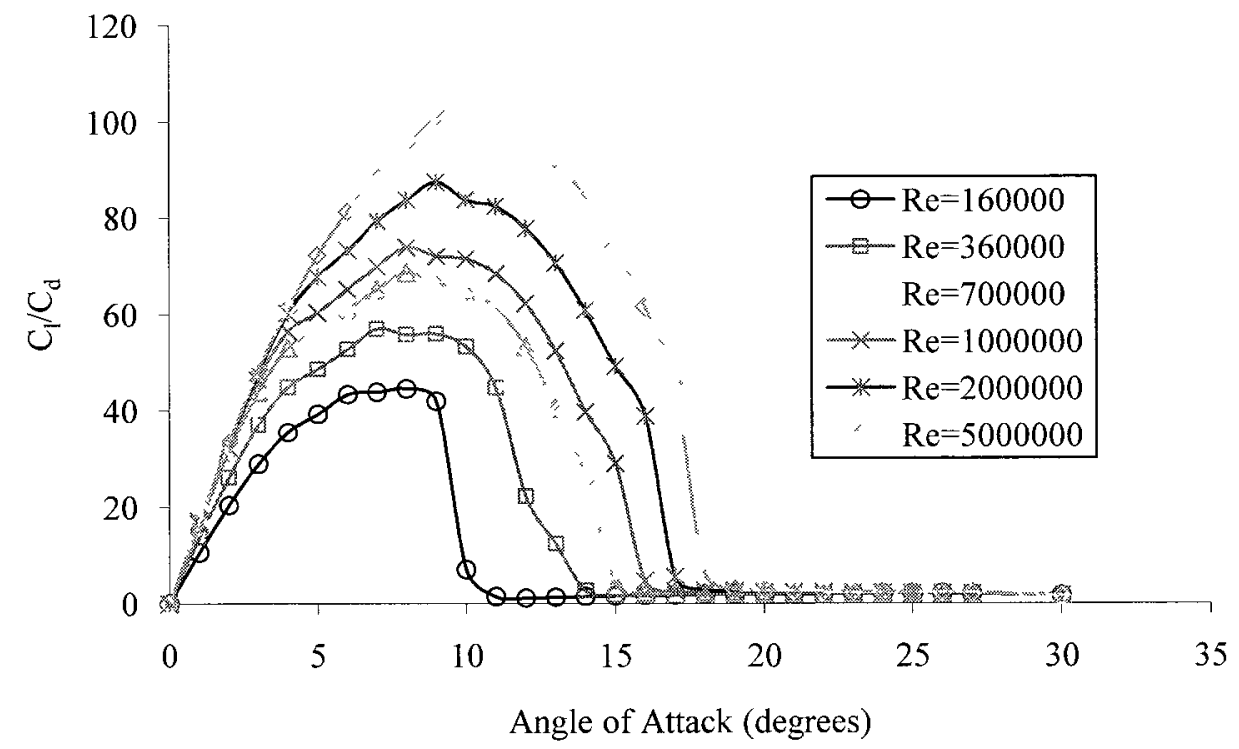

Figure 6.2 Lift to drag ratio for a NACA 0012 foil at various Reynolds numbers (Sheldahl \& Klimas, 1981).
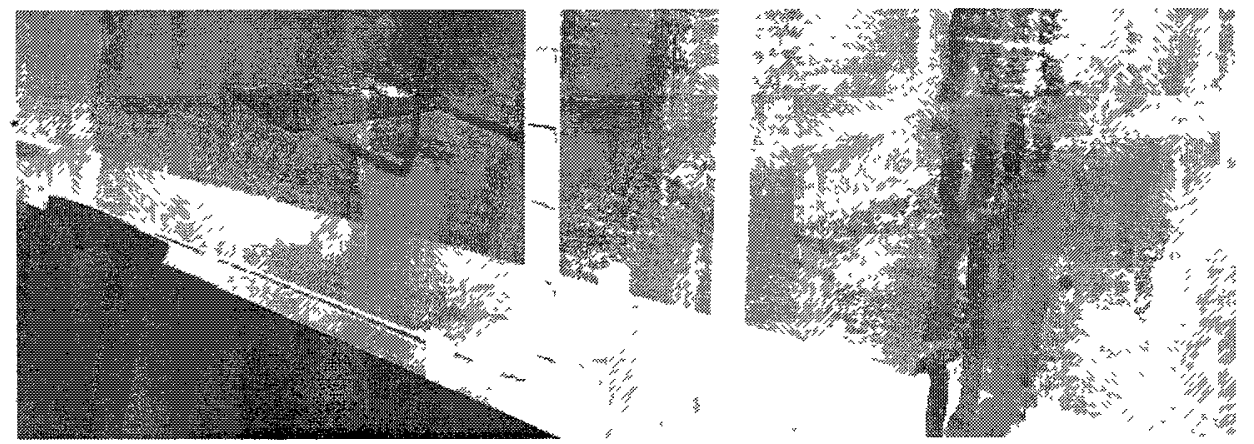

Figure 6.3 Delay of stall experimental setup. Flow is from right to left.
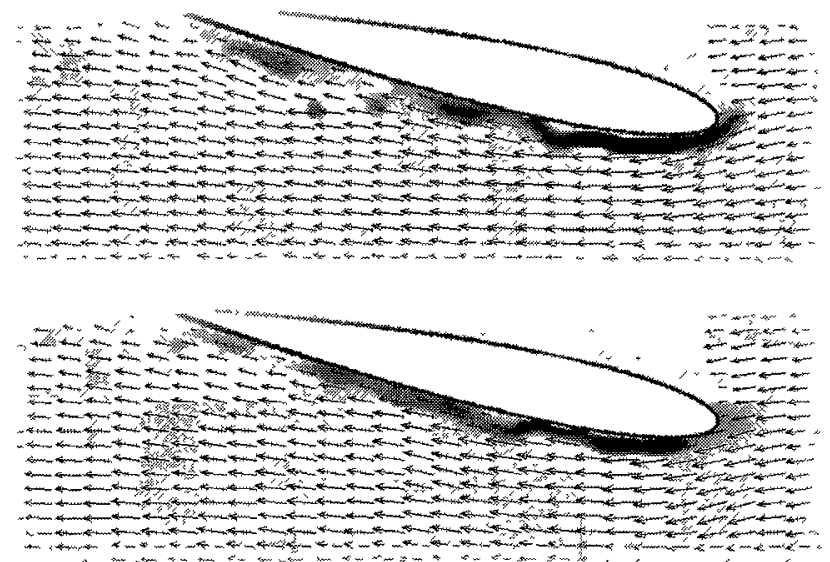

Figure 6.4 Instantaneous velocity vectors for NACA0012 hydrofoil AT $\alpha=9^{\circ}, \operatorname{Re}_{\mathrm{c}}=$ 21,000; Free stream turbulence level of 1\% (top) and 5\% (bottom). Background color indicates in-plane vorticity. 

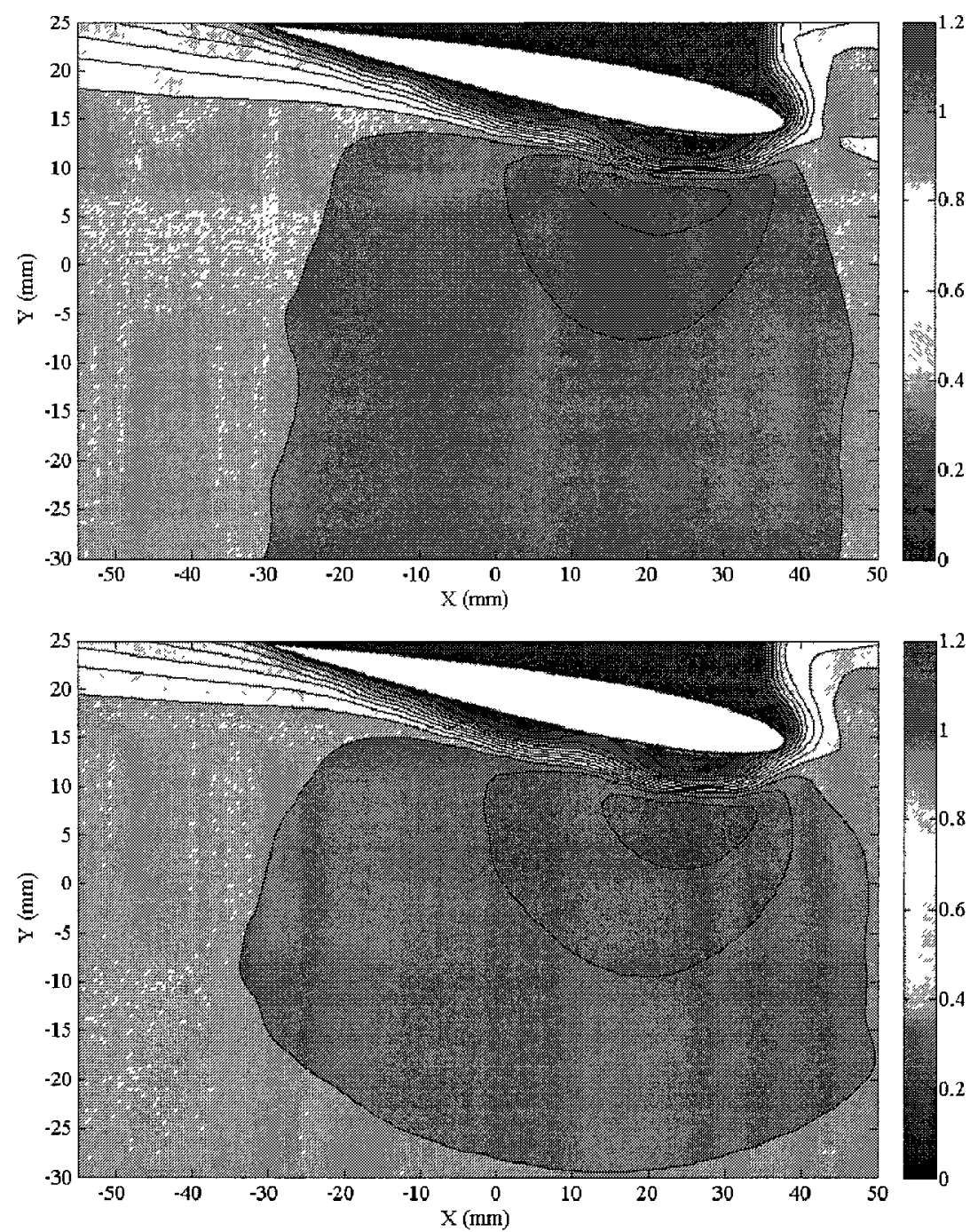

Figure 6.5 Mean velocity contours normalized by free stream velocity with (bottom) and without (top) grid turbulence.

\subsection{GHT Performance in Grid Turbulence}

The hydrokinetic turbine test bed was expanded to enable testing of turbines in grid turbulence. A turbulence generating grid was fabricated with a $36 \%$ solidity and mesh size of 2.5 inches $(6.4 \mathrm{~cm})$, placed 10 mesh widths upstream $(10 M)$ from the edge of the turbine blade sweep, shown in Figure 6.6. The grid wire diameter Reynolds number at 1 $\mathrm{m} / \mathrm{s}$ is about 12,000 , and the turbine location is between $10 M$ and $25 M$ downstream. A Nortek Vectrino+ Acoustic Doppler Velocimeter (ADV) was mounted approximately 5 
mesh widths downstream from the grid, measuring velocity at a sample rate of $200 \mathrm{~Hz}$. Average turbulence intensities in the cross and streamwise directions were measured to be $23 \%$ at this location, confirming the finding of (Groth \& Johansson, 1988) that turbulence anisotropies quickly subside downstream of high Reynolds number, supercritical grids (i.e., the anisotropy measure $v_{r m s} / u_{r m s}$ quickly converges to one). Upon further comparison to the results of (Groth \& Johansson, 1988), the turbulence intensities at the front edge of the turbine sweep (10M from the grid) should be approximately halved, or $11 \%$. The (undisturbed) grid turbulence would then experience a power law decay as described by Batchelor \& Townsend (1948). However, here the decay of turbulence intensity is more complicated due to the induction exerted by the turbine, as well as the interaction with turbine blades, and will be less rapid than that reported by Groth \& Johansson (1988).

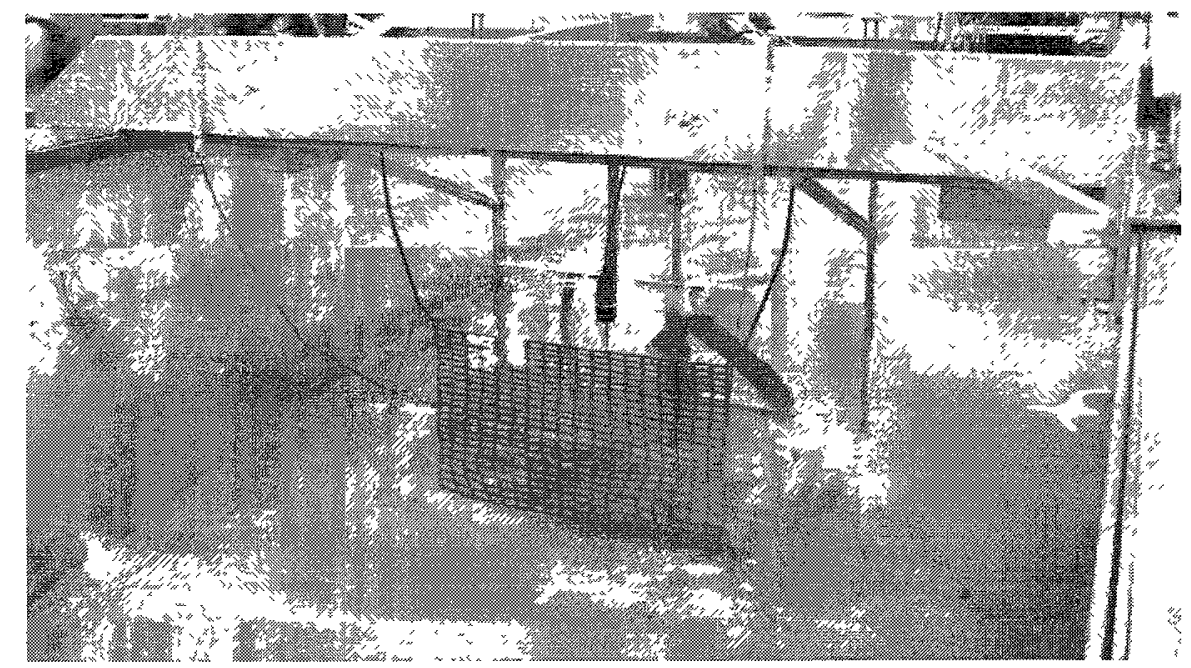

Figure 6.6 UNH-CORE hydrokinetic turbine test bed with grid turbulence generator installed.

In order to calculate tip speed ratio, drag coefficients, and power coefficients to be compared with the steady flow case, a mean post-grid flow velocity must be estimated; 
otherwise, all values will be much lower than the steady case. This is done by measuring velocity with the ADV with only the grid installed, the results of which showed an equivalent free stream velocity behind the grid of 0.88 times the carriage velocity. Experimental error is estimated by adding the ADV's error and the carriage speed error in quadrature, hence the slightly higher average experimental errors compared with the steady case.

Figure 6.7 shows power coefficient plotted versus tip speed ratio for the GHT in grid turbulence. The trends look similar to the steady case, with about the same maximum performance, but with an overall shift to lower tip speed ratios. Maximum tip speed ratio is lowered slightly while minimum tip speed ratio is decreased with no noticeable decrease in power coefficient. This suggests that blade stall is being delayed by the presence of grid turbulence. Delay of stall also would result in lower shaft torque variance (at least for straight-bladed turbines) at lower tip speed ratios compared with the non-turbulent case. Turbulence can thus have a favorable effect on CFA turbines. 


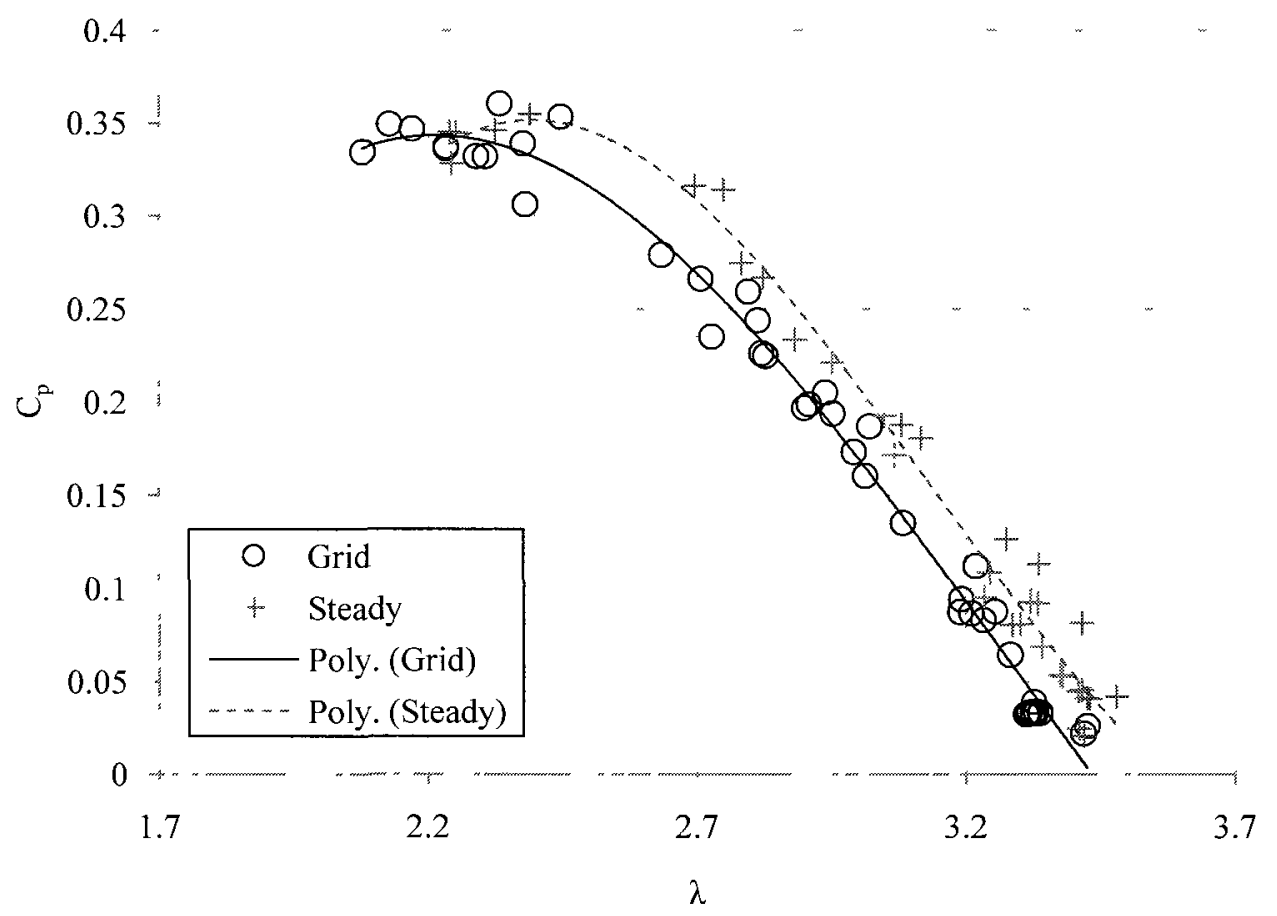

Figure 6.7 GHT power coefficient versus tip speed ratio in grid turbulence for $U=$ $0.8-1.1 \mathrm{~m} / \mathrm{s}$. Average error for $C_{p}=0.02$; for $\lambda=0.05$.

Figure 6.8 shows the GHT's drag coefficient in grid turbulence plotted versus tip speed ratio. A small increase in drag coefficients is observed over the entire range of tip speed ratios compared with the non-turbulent case. This drag increase could explain why the turbine would need to operate at a lower tip speed ratio to lower streamwise induction, allowing the same flow velocity through the turbine, explaining the leftward shift in the power coefficient curve. 


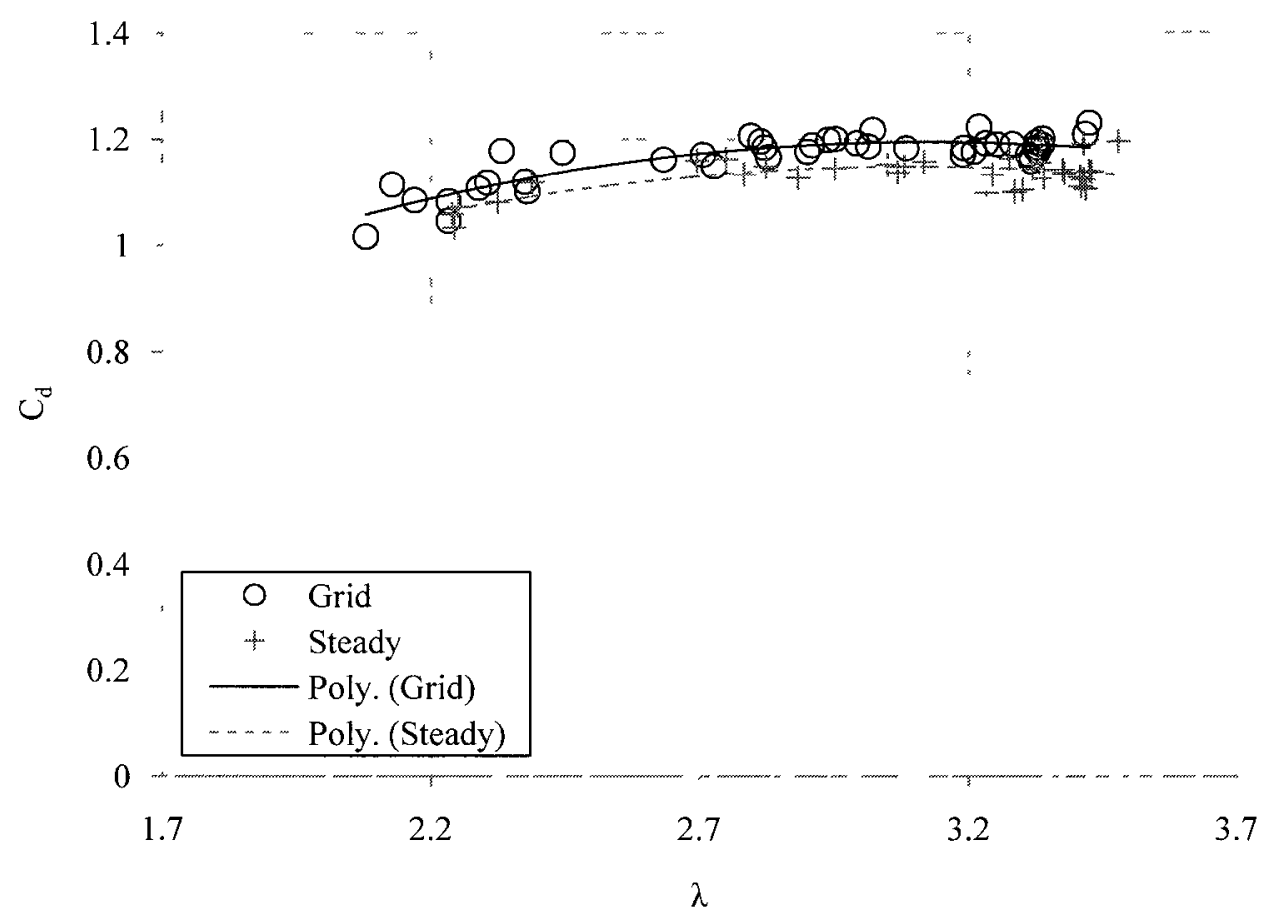

Figure 6.8 GHT drag coefficient versus tip speed ratio in grid turbulence for $U=0.8$ $1.1 \mathrm{~m} / \mathrm{s}$. Average error for $C_{d}=0.007$; for $\lambda=0.05$.

\subsection{GHT Performance in Turbulent Cylinder Wake}

Grid turbulence is approximately isotropic and homogenous, but real world flows have larger coherent structures that originate from complex bathymetry typically found in regions of high tidal current velocities or obstructions such as bridge piers, which will interact with turbine hydrofoils in a more unsteady manner. To make an attempt at a first observation into the effects of larger scale turbulence, GHT performance was measured with a 4.5 inch diameter cylinder wake at various cross stream locations for $U=1.1-1.2$ $\mathrm{m} / \mathrm{s}$. The center of the cylinder was 60 inches or $13 d$ upstream of the center of the turbine. The experimental setup is shown in Figure 6.9. 


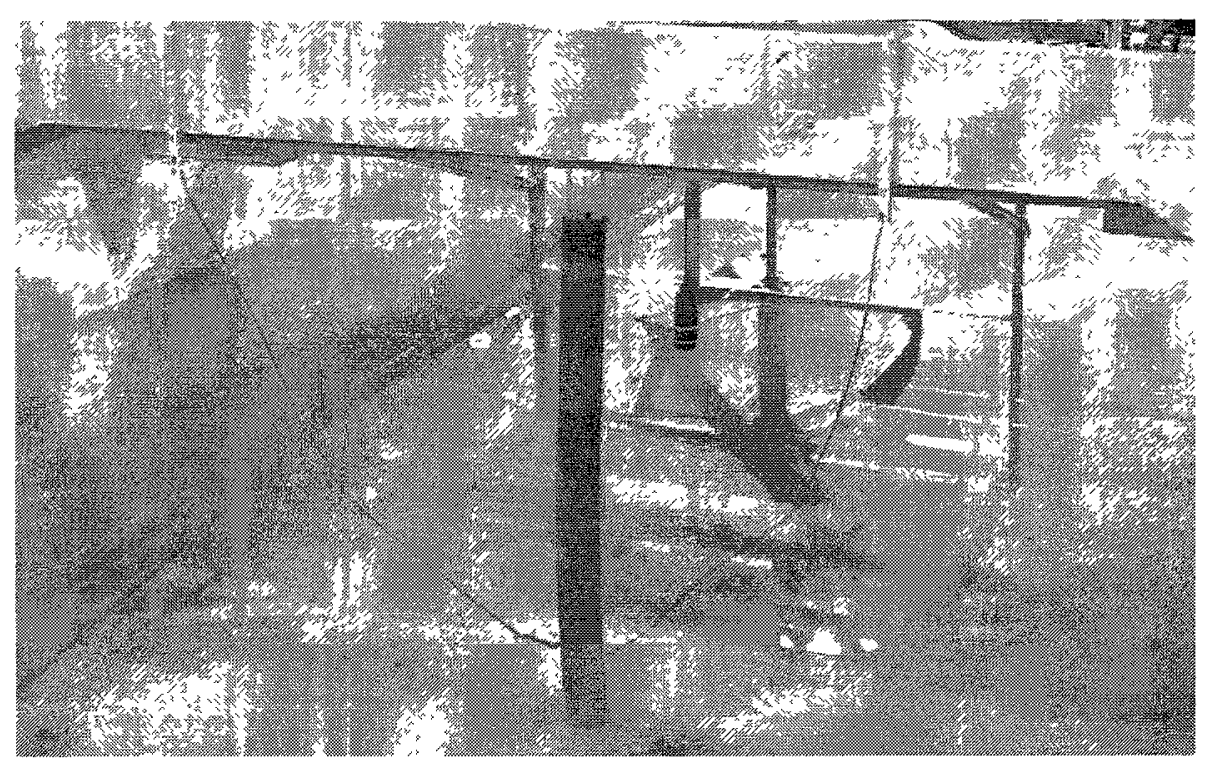

Figure 6.9 UNH-CORE hydrokinetic turbine test bed with cylinder wake turbulence generator installed.

Cylinder flow is mainly characterized by two nondimensional parameters, the Reynolds number with respect to cylinder diameter, $R e_{d}$, and Strouhal number, $S t$. Reynolds number gives a measure of how turbulent the wake is and Strouhal number is a measure of the vortex shedding frequency behind the cylinder as (Zdravkovich, 1997)

$$
f_{\text {shed }}=S t \frac{U}{d} \text {. }
$$

From Zdravkovich (1997), the Strouhal number can be identified to be approximately 1.9 for the range of Reynolds numbers encountered in these experiments. Table 6.1 shows relevant parameters and flow characteristics for the cylinder wake used for these experiments. Figure 6.10 shows a sketch of the experimental setup and cylinder placement coordinate system. Note that for positive values of $Y$, turbine blades are moving toward the cylinder when they are directly downstream, whereas for negative values of $Y$, turbine blades are moving away from the cylinder when directly downstream. 


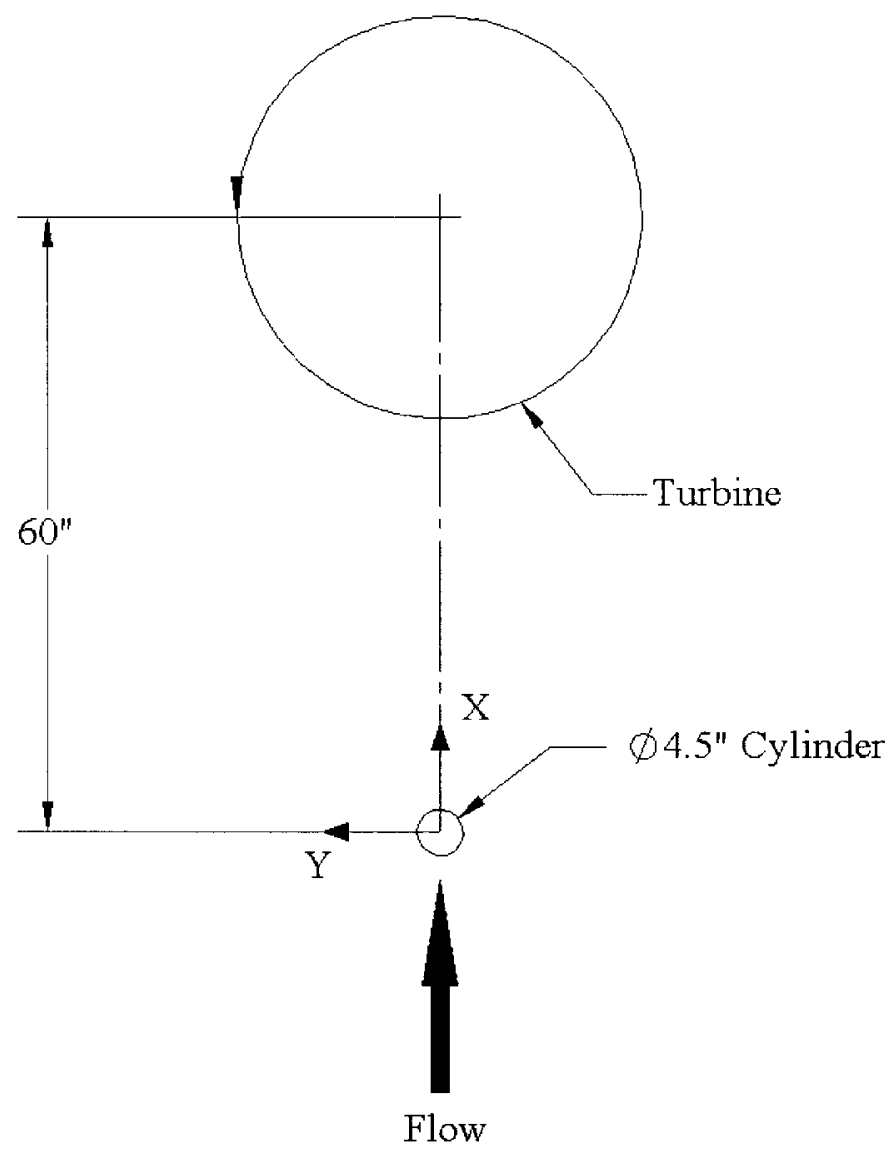

Figure 6.10 Schematic showing cylinder wake experimental setup.

Table 6.1 Cylinder flow parameters.

\begin{tabular}{cc}
\hline$U(\mathrm{~m} / \mathrm{s})$ & $1.1-1.2$ \\
$d(\mathrm{in})$ & 4.5 \\
$\mathrm{Re}_{\mathrm{d}}$ & $13000-14000$ \\
$\mathrm{St}$ & 0.19 \\
$f_{\text {shed }}(\mathrm{Hz})$ & $1.8-2.0$ \\
\hline
\end{tabular}

Figure 6.11 shows the GHT's power coefficient versus tip speed ratio in wake of a cylinder at various cross-stream locations. Note that these coefficients are not calculated with the actual mean velocity taking into account the deficit of the cylinder. 


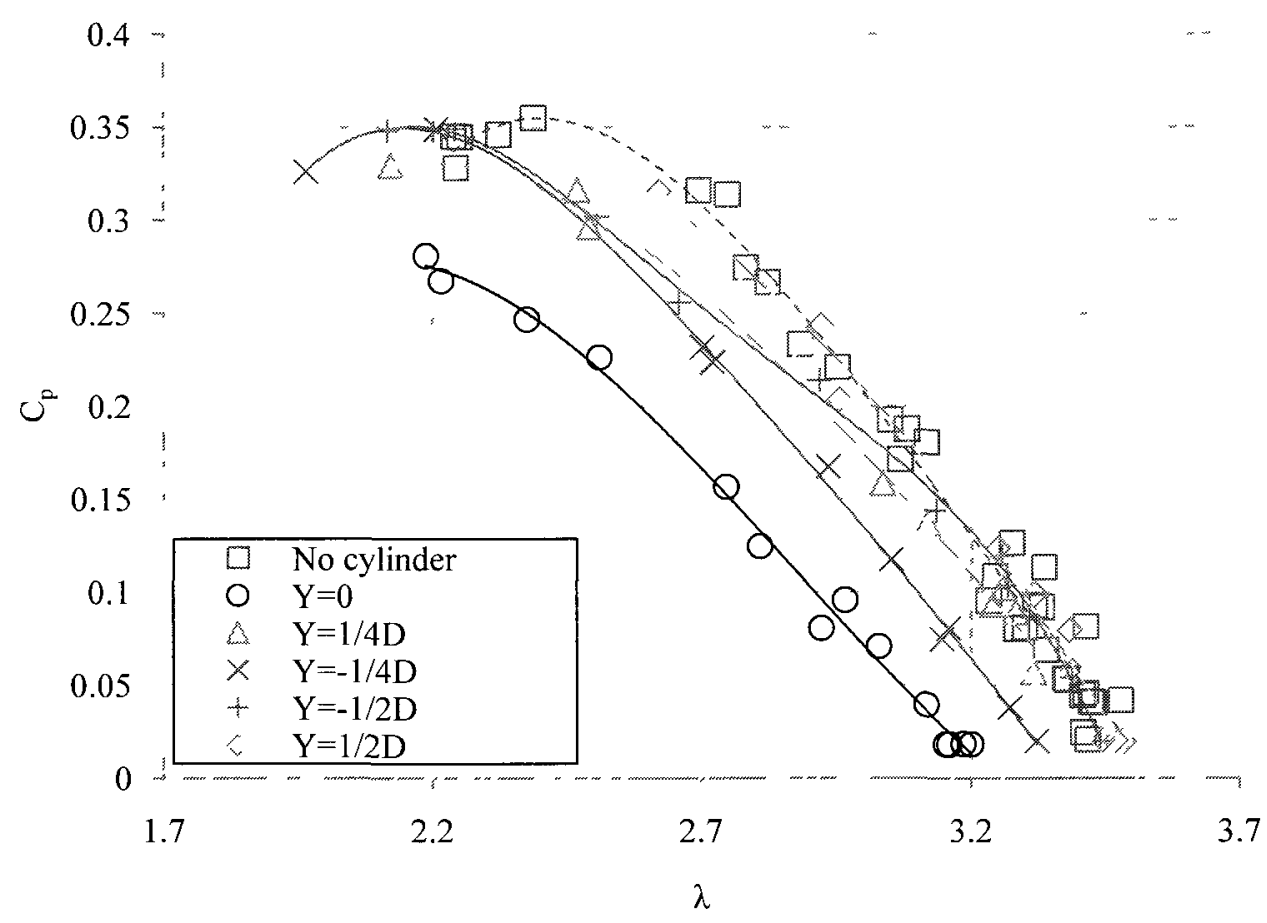

Figure 6.11 GHT power coefficient in 4.5 inch diameter cylinder wake at various cross-stream locations for $U=1.1-1.2 \mathrm{~m} / \mathrm{s}$.

Turbine performance is degraded the most by a significant margin when the cylinder is on center. The turbine stalls near where it would for the uniform flow case, but power coefficients and tip speed ratios above this point are much lower. This can be explained by looking at what the cylinder velocity deficit does. At this angle of rotation, the turbine blade is encountering its highest angle of attack, therefore this is where the majority of hydrodynamic torque is produced during a blade's rotation. With the cylinder on center drag coefficient is also significantly reduced while drag coefficient remains fairly unchanged for other cylinder positions.

At location $Y=1 / 4 D$, performance is degraded more and more as tip speed ratio decreases. At location $Y=-1 / 4 D$, performance is very similar to that of the non-turbulent case, but shifted to lower tip speed ratios. If the velocity deficit of the wake was taken 
into account, power coefficient would be substantially higher. The velocity deficit seems to be reducing velocity at a location where blade stalling would normally occur. At location $Y=-1 / 2 D$, maximum power coefficient seems to be shifted to a lower tip speed ratio, though at higher tip speed ratios power coefficients are comparable to the steady case. At location $Y=1 / 2 D$, performance is comparable to that in uniform flow except for the early stalling at a tip speed ratio of 2.6. This hints at the possibility of the cylinder wake causing low frequency variance in the hydrodynamic torque, therefore instability in the passive loading scenario, similar to the experiments in progressive waves.

Figure 6.12 shows GHT drag coefficient plotted versus tip speed ratio. Data for all cylinder locations seem to be comparable to the uniform flow case except when the cylinder was directly in line with the turbine axis $(Y=0)$. This was likely due to the mean velocity deficit of the cylinder wake reducing fluid velocity at the turbine.

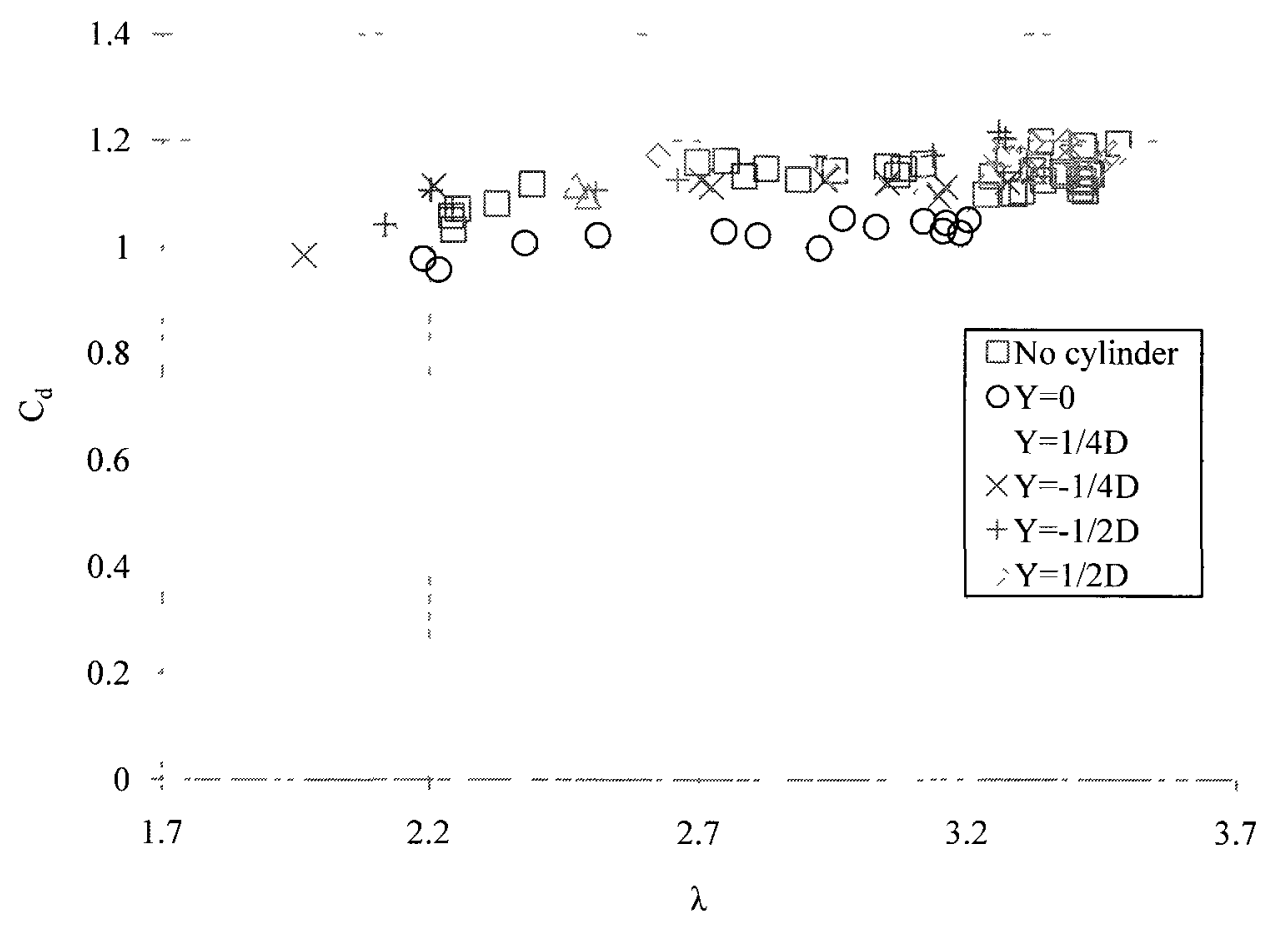

Figure 6.12 GHT drag coefficient in 4.5 inch diameter cylinder wake at various crossstream locations for $U=1.1-1.2 \mathrm{~m} / \mathrm{s}$. 


\subsection{Conclusions - Performance in Turbulence}

In grid turbulence, the maximum power coefficients measured for the GHT were not much different from the non-turbulent case, though the lower tip speed ratio limit was expanded, which is likely due to the turbulence delaying stall of the turbine blades. Grid turbulence increased measured drag coefficients for all tip speed ratios tested. It appears that this increase in drag in grid turbulence changes the induction characteristics of the rotor, shifting operation to lower tip speed ratios, or decreasing performance at higher tip speed ratios relative to the non-turbulent case.

It is unclear which of the observed effects are from the cylinder wake fluctuations and which are merely from the change in mean velocity profile. Further investigation is required, possibly with the help of CFD simulations. Experimentally, PIV measurements in the tow tank would help greatly to visualize any sort of interaction between blades and coherent structures in the cylinder wake, and how these differ for different tip speed ratios. These interactions could be vortex-foil or even vortex-vortex interaction between those created by the cylinder wake and those produced by turbine blades in dynamic stall. A summary of vortex-body interaction dynamics can be found in Rockwell (1998).

From a practical standpoint, these cylinder wake experiments have implications for where to place structural members if they need to be in the flow path. For example, if an array of turbines is to be placed somewhere behind bridge pilings, support struts, etc. This preliminary investigation helped formulate new questions that need to be addressed. Further research is required, with different upstream turbulence generators, position/spacing, and flow measurement/visualization. 


\section{Conclusions and Future Work}

\subsection{Conclusions}

A test bed for cross-flow axis hydrokinetic turbines was developed using the UNH Tow \& Wave Tank. This test bed allows measurement of torque, RPM, and streamwise drag for cross-flow axis (CFA) turbines with up to $1.3 \mathrm{~m}^{2}$ frontal area at speeds up to $1.5 \mathrm{~m} / \mathrm{s}$ with the tow tank's towing mechanism in its present condition. For steady uniform flow, tip speed ratios can range between the no load and peak torque operating conditions, but no lower, due to the passive loading setup. The test bed can also test turbine performance in propagating surface waves along with grid and cylinder wake turbulence.

The test bed was used to measure the performance of two different helical crossflow axis hydrokinetic turbines, one spherical (LST) and one cylindrical (GHT). A blockage correction based on a single streamtube model was applied to the data. The GHT is shown to be a more effective converter of fluid kinetic energy in the relatively low blockage tow tank environment. The performance difference between the GHT and LST can be attributed partially to its higher solidity and smaller average radius. The solidity also explains the lower operating tip speed ratios of the LST. Drag coefficients for both turbines remain fairly constant regardless of power extraction and the LST is in general a lower drag device.

Using one dimensional momentum theory, an expression for estimating linear kinetic exergy efficiency - a Second Law efficiency - was derived. Peak kinetic exergy efficiencies for both the GHT and LST are calculated to be around $90 \%$. This shows that 
although the LST in general operates at lower power coefficients, its lower drag means it is not "wasting" any more kinetic power than the GHT.

GHT performance was then tested while towed against $0.15 \mathrm{~m}$ high propagating waves of various periods. A significant increase in power and drag coefficients was observed. However, longer period waves prevent stable operation at lower tip speed ratios in the passive loading configuration.

A simple two-dimensional computer model was developed to attempt to explain measured performance. An approximate linear relationship between streamwise induction and tip speed ratio was chosen to best fit the experimental data. This model seems to sufficiently explain power and drag coefficients based on static foil performance data. When the streamwise wave-induced velocity fluctuation, calculated from linear small amplitude wave theory, is added to the model's free stream velocity, foil forces can be shown to increase the shaft torque variance significantly at low frequencies, explaining the inability to operate at lower tip speed ratios. This variance is due to the wave velocity augmenting blade angle of attack past its stall angle for some rotations. This also shows the need for a feedback controlled load system to keep tip speed ratio at a desired value. The simple model cannot, however, explain the increase in turbine power output.

In isotropic homogeneous turbulence, the GHT's power coefficient is shown to be similar to the non-turbulent case, but shifted to lower tip speed ratios. Drag coefficients are also higher, suggesting streamwise induction is increased, hence the tendency to operate a lower tip speed ratios. The lowest operable tip speed ratio is expanded slightly, most likely due to the turbulence delaying blade stall. 
When operating in a cylinder wake with a diameter on the order of the turbine blade chord length 13 diameters upstream of the center of the turbine, turbine performance is most negatively affected when the turbine is directly on center downstream of the cylinder. Other locations have less of a negative effect, but will generally shift the tip speed ratio of optimal performance to lower values. In the experiments conducted to date the effects from the turbulence in the cylinder wake and the effects from the mean velocity deficit could not be separated. This question could be first investigated by adding cylinder wake mean velocity profiles to the previously developed 2D model.

Overall, waves and turbulence were shown to have significant effects on turbine performance. This shows the need for more detailed data regarding turbine operation and the need to better characterize potential deployment sites. In the future, it is recommended that a velocity spectrum be used as input to a prediction model, instead of simply a constant free stream.

\subsection{Future Work}

Although this study somewhat sacrificed depth for breadth, it has opened up some new avenues and questions for investigation.

The mechanical load application of the turbine test bed built for the experiments described in this thesis allows only for the operation of the turbines at tip speed ratios above those of peak torque. Feedback control of turbine tip speed ratio is highly desirable for future testing. Using a motor with closed-loop feedback control of rotational speed would allow prescription of tip speed ratio, changing turbine loading from a passive to an 
active load. This would also allow for the acquisition of higher resolution data from the smaller increments of tip speed ratio.

Regarding experimental setups, for any future testing of these devices the speed control and dynamics of the tow carriage must be addressed. It may be helpful to allow programmed waveforms of speed (sine, triangle, etc) to observe how the turbine reacts to unsteady inflows. Also, a new physical model CFA turbine should be developed with flexibility and modularity in mind. This means allowing for ease of changing blades to test different profiles (including cambered profiles), non-uniform blade spacing, helical versus straight blades, removing the center shaft, allowing for blade pitch adjustments, etc. It would also be useful to build a turbine model and instrumentation to record blade position, tangential and normal forces, and possibly even include pressure taps to determine angle of attack. Better flow measurement/visualization, e.g. PIV or an ADV array, would greatly help with understanding fluid flow through the rotor, also allowing a more detailed look at the effects of turbulence. Using PIV to observe the fluid flow through the turbine underneath a progressive wave will also help explain the increase in turbine power output.

For the experiments reported here, only a simple blockage correction based on a streamtube model was used. While the observed deformation of the free surface during experiments was very small, for future experiments a more sophisticated correction including a free surface deformation correction should be investigated. CFD studies could also be helpful in estimating blockage effects. Further, it would be interesting to see how the calculated kinetic exergy efficiencies change for different blockage scenarios. 
Regarding optimization of CFA turbines, obtaining maximum power from one of these devices seems to be a balance between keeping angle of attack high (to maximize $\beta$ ), avoiding stall (to maximize lift to drag ratio), while rotating at high angular velocity, but not so fast as to obstruct too much flow. For lower solidity devices, induction is lower, therefore the angle of attack at which stall (and also maximum power coefficient) occurs will be reached at a higher tip speed ratio. However, lower solidity means relatively less blade surface area, and therefore hydrodynamic torque. According to the 2D model developed, streamwise induction in the GHT used in this study appears to be high. From 1D momentum (Betz) theory, the power coefficient would be optimized at an induction factor of $1 / 3$. Two ways for lowering induction are hypothesized: lowering solidity or lowering tip speed ratio. Lowering tip speed ratio is not a good option since it lowers rotational speed, thereby lowering power output, and may increases angle of attack oscillations to stall. A lower solidity turbine with thicker blades, having higher stall angles, may improve performance. For equal solidity, it has been shown in experiments that a one-bladed device performs best in terms of average power output, while a two-bladed device is not far behind, and a three-bladed turbine performing significantly worse (Shiono, Suzuki, \& Kiho, 2000). It's possible the increase in performance is due to higher Reynolds number from longer chord lengths in the lower blade number cases. Though a one-bladed Darrieus device would surely have issues with large torque pulsations, a one-bladed GHT may allow less torque pulsation at the shaft, enabling practical operation. These considerations can be tested with a new physical model, working towards reaching the maximum performance of a CFA hydrokinetic turbine. 
One question that remains is whether or not the GHT is a higher performing device compared to a Darrieus turbine of identical solidity, number of blades, and blade profile. The easier self starting and less shaft torque ripple of the Gorlov is one advantage, but this could be offset by the extra manufacturing cost of helical blades. Also, the high transient forces in allowing to self start may mean it's preferable to start manually anyway to prevent damage to the mounting structure. According to one computational study, there seems to be no difference in average power output between a Gorlov versus a Darrieus turbine, just a difference in how torque varies at the shaft (Winchester \& Quayle, 2009). Furthermore, the helical blades of the GHT do not eliminate the unsteadiness in hydrodynamic forces on the blades, but cause these forces to move up and down a blade, possibly leading to blade fatigue, despite the reduction in shaft torque ripple. Blade end effects could be one way the GHT differs. The swept blades may behave similarly to swept aircraft wings, avoiding the induced drag from tip vortices. On a related note, for a helical blade, flow may be different from that of a straight blade due to pressure gradients along the blade caused by differences in lift.

Many ideas for novel experiments have arisen from the initial observations from the experiments reported in this thesis. Will the effects of waves change when the tow direction is the same as the direction of wave propagation? How would different wave height distributions, akin to what would be seen in real world tidal flows, affect turbine performance? What are the effects of waves propagating in the cross-stream direction? How will performance be affected by a transverse cylinder wake? Different grid positions? Would some type of flexible blade allow higher performance through manipulation of vorticity present in the flow? Lastly, it would be interesting to see if the 
effects of different types of waves and turbulence add "linearly?" In other words, will the effects add and subtract independently of each other? 
This page intentionally left blank. 


\section{References}

Amandolese, X., \& Szechenyi, E. (2004). Experimental Study of the Effect of Turbulence on a Section Model Blade Oscillating in Stall. Wind Energy, 267-282.

Anderson, J., Streitlien, K., Barrett, D., \& Triantafyllou, M. (1998). Oscillating Foils of High Propulsive Efficiency. Journal of Fluid Mechanics, 360, 41-72.

Arndt, R. (1991). Hydraulic Turbines. In J. Gulliver, \& R. Arndt, Hydropower Engineering Handbook. McGraw-Hill.

Bahaj, A., Molland, A., Chaplin, J., \& Batten, W. (2007). Power and Thrust Measurements of Marine Current Turbines Under Various Hydrodynamic Flow Conditions in a Cavitation Tunnel and a Towing Tank. Renewable Energy, 407-426.

Banal, M., \& Bichon, A. (1981). Tidal Energy in France, The Rance Tidal Power Station - Some Results After 15 Years in Operation. Proceedings of the Second International Symposium on Wave and Tidal Energy. Cambridge.

Batchelor, G., \& Townsend, A. (1948). Decay of Vorticity in Isotropic Turbulence. Proc. of R. Soc. London, 539-558.

Beal, D. (2006). Passive Propulsion in Vortex Wakes. Journal of Fluid Mechanics, 549, 385-402.

Beer, F., Johnston, E., \& DeWolf, J. (2006). Mechanics of Materials (4th Edition ed.). New York: McGraw-Hill.

Bose, N., \& Lien, J. (1990). Energy Absorption from Ocean Waves: a Free Ride for Cetaceans. Proceedings of the Royal Society of London, 591-605.

Carlton, J. (2007). Marine Propellers and Propulsion. Oxford: Elsevier.

Darnell, L. (1996). A Towing Carriage for the University of New Hampshire Towing and Wave Making Basin. Master's Thesis.

Darrieus, G. J. (1931). Patent No. 1,835,018. United States of America.

Dean, R., \& Dalrymple, R. (1991). Water Wave Mechanics for Engineers and Scientists (1 ed., Vol. 2). Hackensack: World Scientific.

Deutschman, A., Michels, W., \& Wilson, C. (1975). Machine Design Theory and Practice. New York: Macmillan Publishing Co.

Donners, K., Waelkens, M., \& Deckers, J. (2002). Water Mills in the Area of Sagalassos: A Disappearing Ancient Technology. Anatolian Studies, 1-17. 
Electric Power Research Institute. (2006). Methodology for Estimating Tidal Current Energy Resources and Power Production by Tidal In-Stream Energy Conversion (TISEC) Devices. Retrieved from http://www.epri.com/oceanenergy

Ferreira, C., van Kuik, G., van Bussel, G., \& Scarano, F. (2009). Visualization by PIV of Dynamic Stall on a Vertical Axis Wind Turbine. Experimental Fluids, 97-108.

Fox, R., Pritchard, P., \& McDonald, A. (2009). Introduction to Fluid Mechanics. Hoboken: John Wiley \& Sons.

Fujisawa, N., \& Shibuya, S. (2001). Observations of Dynamic Stall on Darrieus Wind Turbine Blades. Journal of Wind Engineering and Industrial Aerodynamics, 201-214.

GCK Technologies. (2002-2006). Gorlov Helical Turbines, Production for Korea Ocean Research and Development Institute (KORDI) and Testing by KORDI. Internal Reports.

Gorlov, A. (1995). Patent No. 5,451,137. United States of America.

Gorlov, A. (1998). Development of the Helical Reaction Hydraulic Turbine. Washington, DC: US Department of Energy.

Groth, J., \& Johansson, A. (1988). Turbulence Reduction by Screens. Journal of Fluid Mechanics, 139-155.

International Energy Agency. (2010). Key World Energy Statistics 2010. Retrieved from http://iea.org/stats/index.asp

Johnston, A. (2011). Analytical and Numerical Modeling of Performance Characteristics of Cross-flow Hydrokinetic Turbines. Durham: University of New Hampshire.

Kammere. (2007). NOS/NOAA Current Survey of Piscataqua River.

Lee, K., Yum, K., Park, J., \& Park, J. (2009). Tidal Current Power Development in Korea. East Asian Sea Congress. Philippines.

Manwell, J., McGowan, A., \& Rogers, A. (2002). Wind Energy Explained. Hoboken: John Wiley \& Sons.

McAdam, R., Houlsby, G., Oldfield, M., \& McCulloch, M. (2009). Experimental Testing of the Transverse Horizontal Axis Water Turbine. Proceedings of the 8th European Wave and Tidal Energy Conference, (pp. 360-365). Uppsala, Sweden.

McKay, D. (2009). Sustainable Energy - Without the Hot Air. UIT Cambridge.

Oberg, E., \& Jones, F. D. (1959). Machinery's Handbook. New York: The Industrial Press. 
Ocean Renewable Power Company. (2008). Status Report 4: OCGen Prototype Tidal Turbine Generator Unit Demonstration.

ORNL, Sandia NL, NREL. (2011). RFP for DOE MHK Reference Turbine Laboratory Studies, W/Supplements for Horizontal and Vertical Axis Wind Turbines. Oak Ridge National Laboratory.

Panton, R. (1933). Incompressible Flow. Hoboken: John Wiley \& Sons.

Paraschivoiu, I. (2002). Wind Turbine Design With Emphasis on Darrieus Concept. Montreal, Quebec, Canada: Polytechnic International.

Rockwell, D. (1998). Vortex-body Interactions. Annu. Rev. Fluid Mech. , 199-229.

Savonius, S. (1930). Patent No. 1,766,765. United States of America.

Schlabach. (2010). In-Pipe Hydro-Electric Power System and Turbine. Northwest Pipe and Lucid Energy.

Schlabach, R. (2010). Lucid Energy Technologies In-Conduit Hydro Power Testing at Utah Water Research Laboratory. Lucid Energy Technologies.

Sheldahl, R., \& Klimas, P. (1981). Aerodynamic Characteristics of Seven Symmetrical Airfoil Sections Through 180-Degree Angle of Attack for use in Aerodynamic Analysis or Vertical Axis Wind Turbines. Albuquerque, NM: Sandia National Labs.

Sheng, W., Galbraith, R. A., \& Coton, F. N. (2008). Prediction of Dynamic Stall Onset for Oscillatory Low-Speed Airfoils. ASME Journal of Fluids Engineering .

Shiono, M., Suzuki, K., \& Kiho, S. (2000). An Experimental Study of the Characteristics of a Darrieus Turbine for Tidal Power Generation. Electrical Engineering in Japan, 3846.

Siegel, S., Jeans, T., \& McLaughlin, T. (2010). Intermediate Ocean Wave Termination Using a Cycloidal Wave Energy Converter. 29th International Conference on Ocean, Offshore, and Arctic Engineering.

Swalwell, K. E., Sheridan, J., \& Melbourne, W. H. (2001). The Effect of Turbulence Intensity on Stall of a NACA 0021 Aerofoil. 14th Australasian Fluid Mechanics Conference, (pp. 941-944). Adelaide, Australia.

Taylor, J. (1997). An Introduction to Error Analysis. Sausalito, CA: University Science Books.

Testing the Waters with Tidal Energy. (2010, December). Scientific American . 
United States Department of Energy. (2010). Annual Energy Review 2009. Washington, DC.

Vanek, F., \& Albright, L. (2008). Energy Systems Engineering. New York: McGrawHill.

Williams, G. E. (2000). Geological Constraints on the Precambrian History of Earth's Rotation and the Moon's Orbit. Reviews of Geophysics, 37-60.

Winchester, J. D., \& Quayle, S. D. (2009). Torque Ripple and Variable Blade Force: A Comparison of Darrieus and Gorlov-type Turbines for Tidal Stream Energy Conversion.

Proceedings of the 8th European Wave and Tidal Energy Conference, (pp. 668-676). Uppsala, Sweden.

World Energy Council. (2007). 2007 Survey of Energy Resources. London: World Energy Council.

Zdravkovich, M. M. (1997). Flow Around Circular Cylinders Vol 1: Fundamentals. New York: Oxford University Press. 


\section{Appendix A}

\section{A.1 Tips for Test Bed Operation and Lessons Learned}

It was determined that the ideal test bed operation is as follows:

- Start data acquisition.

- Person onboard carriage begins turbine rotation manually.

- Start carriage motion.

- Person onboard carriage applies brake when carriage is around $1 / 3$ the length of the tank.

- Send the command to stop the carriage with about $30 \mathrm{ft}$ left until the wave maker to allow adequate coasting distance.

- Stop data acquisition.

- Adjust brake stop screw. Do this in increments of $1 / 6$ of a turn from no load up until the turbine stalls.

One important lesson learned is that drag force on a rotating turbine is much greater than when stopped. As described earlier, this can cause high transient forces in the tow mechanism if the turbine is allowed to self-start.

\section{A.2 Selected Design Calculations}

Since the weight of the turbine, shaft, torque transducer, and couplers would need to be supported by the horizontal foil members of the main frame assembly, bending stresses had to be checked for safety. It was assumed that the full load would need to be supported by the lower horizontal only and that this beam would be fixed at both ends. Since there 
were two through holes and a small area milled flat near the center of the foil, this is the section of lowest area moment of inertia, calculated to be $0.199 \mathrm{in}^{4}$ in Solidworks, and is highlighted in Figure A.1. The bending stress at the center for a beam fixed at both ends with a point load at the center is given by

$$
\sigma_{\max }=\frac{W L}{8 I},
$$

where $W$ is the load, $L$ is the length of the beam, and $I$ is the area moment of inertia (Oberg \& Jones, 1959). The maximum deflection at the center of the beam is given by

$$
\delta_{\max }=\frac{W L^{3}}{192 E I},
$$

where $E$ is the modulus of elasticity of the material (Oberg \& Jones, 1959).

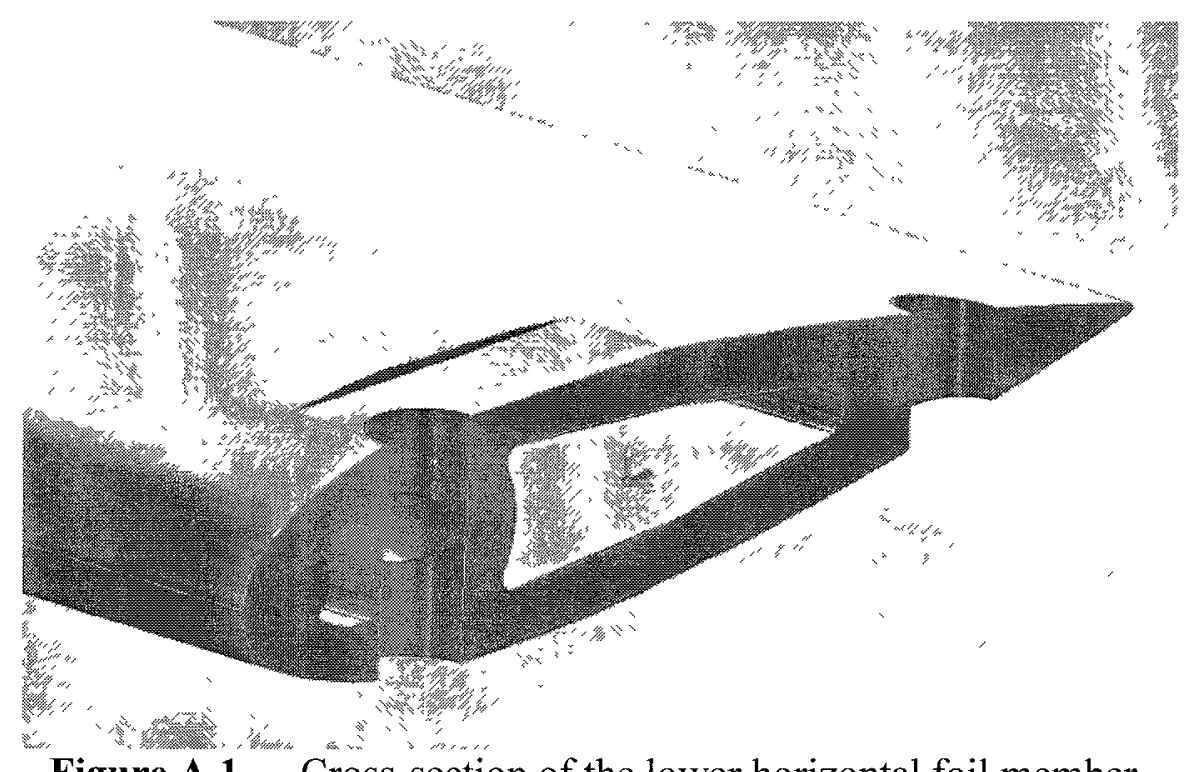

Figure A.1 Cross-section of the lower horizontal foil member.

Using Equation (A.1), a load of $134 \mathrm{lbf}$ (estimated weight of the components in air computed by Solidworks), and the beam length of 65.5 inches, the predicted maximum stress in the beam was approximately $5,500 \mathrm{psi}$, which is well below the 35,000 psi yield 
stress for 6061-T6 aluminum (Beer, Johnston, \& DeWolf, 2006). Similarly, using Equation (A.2), the predicted maximum deflection was approximately 0.1 inches, which was considered acceptable for this application.

\section{A.3 Additional Instrumentation and Component Details}

Experimental error estimates given in Table 4.1 were calculated using the manufacturer's published accuracy specifications from their respective datasheets. Table A.1 shows each source of error considered in the calculations. Figures A.2-A.6 are the individual factory calibrations used for data processing. Note that these sheets show each device to be within factory specifications for accuracy, illustrating how the individual component errors were conservative estimates.

Table A.1 Individual component error considered.

\begin{tabular}{ccc}
\hline Quantity & Error & Notes \\
\hline Speed encoder dia. & $.001 \%$ & Measured with calipers \\
Sentran ZB load cell & $0.125 \mathrm{lbf}$ & $500 \mathrm{lbf}$ capacity \\
Interface T8 torque trans. & $1 \mathrm{Nm}$ & $200 \mathrm{Nm}$ capacity \\
Dataforth DSCA45 freq. cond. & $0.1 \% \mathrm{span}$ & $500 \mathrm{~Hz}$ span for RPM, $2.5 \mathrm{kHz}$ span \\
& & for carriage speed \\
Dataforth DSCA38 exc. & $0.006 \mathrm{~V}$ & $10 \mathrm{~V}$ nominal output \\
Dataforth DSCA38 amp. & $0.06 \% \mathrm{span}$ & $\pm 10 \mathrm{~V} \mathrm{span}$ \\
Hydrometer (water density) & $2 \mathrm{~kg} / \mathrm{m}^{3}$ & $1002 \mathrm{~kg} / \mathrm{m}^{3}$ measured \\
USB-6211 DAQ & $2.690 \mathrm{mV}$ & Device in $\pm 10 \mathrm{~V}$ range mode \\
Vectrino+ velocity & $1 \% \mathrm{of}$ & \\
& meas. val. & \\
& $\pm 0.001 \mathrm{~m} / \mathrm{s}$ & \\
\hline
\end{tabular}

Table A. 2 contains part numbers for selected purchased items. These may be useful if replacement is necessary. 
Table A.2 Part numbers for selected purchased items.

\begin{tabular}{cc}
\hline Item & Part Number \\
\hline Brake rotor & NAPA NB 4886576 \\
Brake caliper & NAPA CAL SE5157A \\
Upper shaft bearing & NAPA PGB PBR930252 \\
Brake hose & Summit 634510 \\
Brake master cylinder & Wilwood 260-6766 \\
Precision bearing slides & McMaster 6709K11\& 6709K301 \\
Lower (submerged) shaft bearings & McMaster 3756T140 \\
\hline
\end{tabular}




\section{Latt (ant bench)}

\section{PRODUCT CERTIFICATION}

\section{SENTRAN}

\begin{tabular}{|c|c|}
\hline MODEL: & $203-500 \cdot 000$ \\
\hline SERLAL NO: & 984924 \\
\hline CAPACT: & $500 \mathrm{LB}$ \\
\hline $\begin{array}{l}\text { COMBINED ERROK } \\
(0 \mathrm{FSO})\end{array}$ & 0.03 \\
\hline WIRING COOE: & 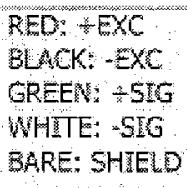 \\
\hline DABE: & $05 / 03 / 2010$ \\
\hline
\end{tabular}

\begin{tabular}{|c|c|}
\hline FULL SCMLE OUTPUT $(\mathrm{m} / \mathrm{N})$ : & 2,997 \\
\hline ZERO QALANCE (\% FSO) & $<1.0$ \\
\hline NON-REPEATABILTIY (O FSOY) & $<0,01$ \\
\hline CPEE (\% Reading in 30 rin): & 20,03 \\
\hline MAX EXCTATLOU U, AC OR DCI: & 15.0 \\
\hline COMEENSATED TEME RANGE $(O C)$ & $.10 T 040$ \\
\hline OPRRATNG TEMP RANGE YOF: & 2012060 \\
\hline INSULATION RESISTANCE (WO O SOVDC): & $>5000$ \\
\hline EAROUEIRIC EFFECT: & NIL \\
\hline INPIIT RESISTANCE $(\Omega)$ & 385 \\
\hline OUTPUT RESISTANCE $(\Omega)$ & 350 \\
\hline TEME EFFECT ON OUTPUT (WREdIng OC): & $<0.0015$ \\
\hline TENP EFFECT ON ZERO (9S $550 / O \mathrm{C}:$ & $<0,0027$ \\
\hline SAFE LOAD IIMT (O CAPACTTO: & 150 \\
\hline
\end{tabular}

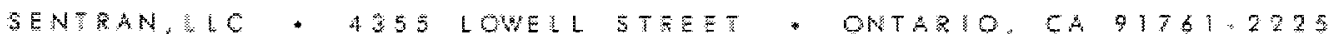
YOICE:19081605-154L. FAR:1909)

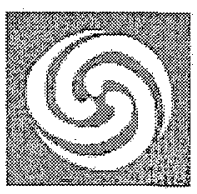

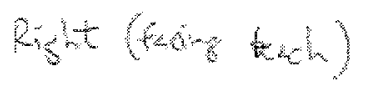

\section{PRODUCT CERTIFICATION}

\begin{tabular}{|c|c|}
\hline MODEL: & $203-500-000$ \\
\hline SERIAL NO: & 984923 \\
\hline CAPACRT: & $500 \mathrm{LB}$ \\
\hline $\begin{array}{l}\text { COMBINED ERROR } \\
(\% \text { FSO): }\end{array}$ & 0,03 \\
\hline WIRING COQE: & 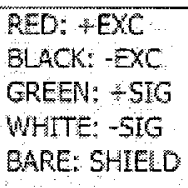 \\
\hline DATE: & $05 / 03 / 2010$ \\
\hline
\end{tabular}

\begin{tabular}{|c|c|}
\hline FULL SCALE OUTPUT $/ \mathrm{mVNV}$ & 2.999 \\
\hline ZERO BALAMCE (\% FSO): & $<1.0$ \\
\hline NON-REPEATABLLTY (\% F5O): & $<0.01$ \\
\hline CREEP (W Reading Im $30 \mathrm{~min}$ ) & $<0.03$ \\
\hline MAX EXCITATION Y AC OR DCL & 150 \\
\hline COMPENSATEO TEYP RANGE (OO) & 107040 \\
\hline OPERATING TEMP RANGE $9 \mathrm{CV}$ & 201080 \\
\hline INSLLATION RESISTANCE (MD O SOVDC): & $>5000$ \\
\hline BAROMETRIC EFFES: & NLL \\
\hline NAPUT RESISTANCE $(\Omega):$ & 385 \\
\hline OURURKESSTANCE (O): & 350 \\
\hline TERP EFEECT ON OUTPUT OFReAing $O C$ : & $\approx 0.0015$ \\
\hline TENP EFFEOT ON $\angle E R O 1 \%$ FSO $/ O \mathrm{C}$ & 50,0027 \\
\hline SAFE $\angle O A D ~ \triangle M T(\%$ CAPACTRY) & 150 \\
\hline
\end{tabular}

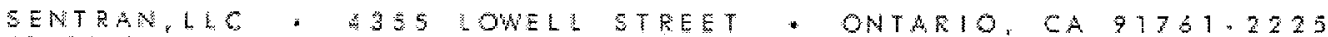

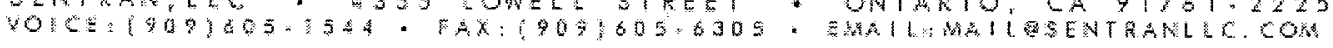

Figure A.2 Factory calibration sheets for left (top) and right (bottom) load cells. 


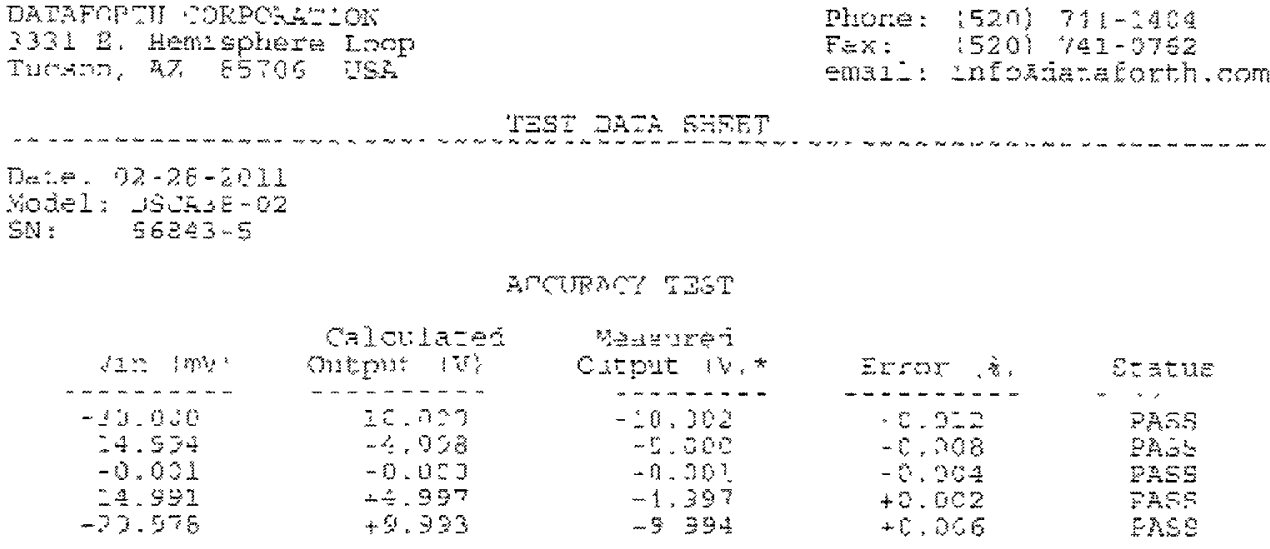

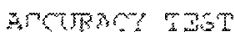

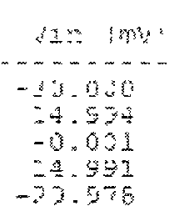

\begin{tabular}{|c|c|c|}
\hline Copult ik, & $525: 5 x$ & $2=\vec{x}+3$ \\
\hline$\ldots \ldots+\cdots$ & $\ldots \ldots \ldots$ & $\cdots$ \\
\hline$-\therefore 0,30$ & $=6.2=$ & $P \pi 58$ \\
\hline-3.35 & -8.308 & $9 a^{2}=$ \\
\hline$-n-36 \%$ & $-5,254$ & 235 \\
\hline-1.397 & +3.002 & $=250$ \\
\hline-5394 & 25,355 & 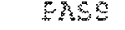 \\
\hline
\end{tabular}

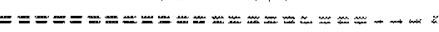

6U9p 15 yom

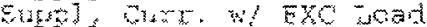

gxo2

DxF, losi Remblat:ov

उद.

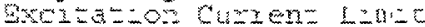

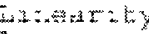

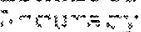

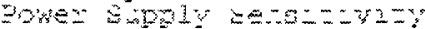

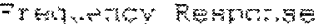

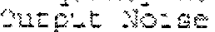

7 वर⿵人一

$2 x-30 t$
GUA TES REMTIS

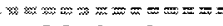
20. $\Pi \pi$

$48.2 \mathrm{~m}$

$30.00 \%$

$-x, 5$ का

$=\bar{x}+{ }^{2}$

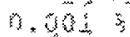

$-9 \times 3=2$

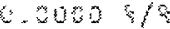

2. 2 . $\mathrm{CD}$

an in:men

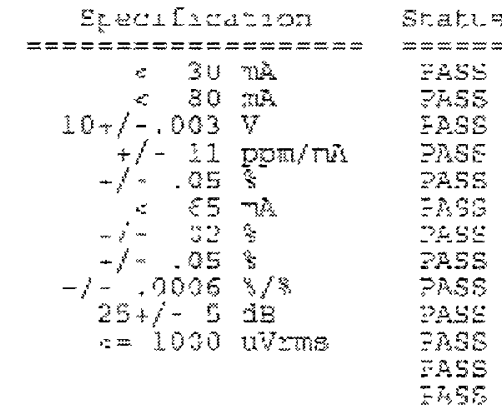

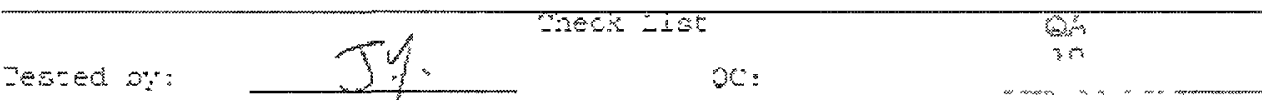

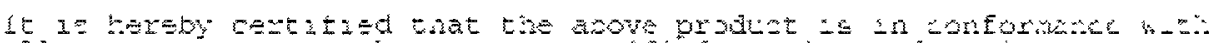

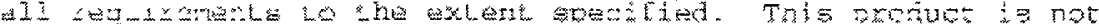

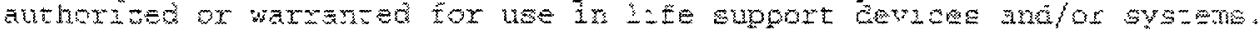

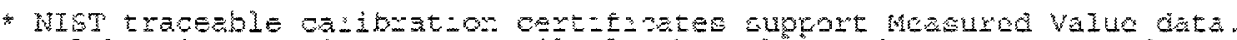

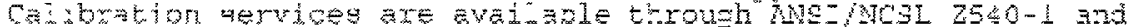

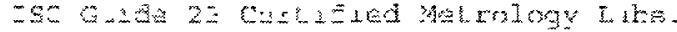

Figure A.3 Factory calibration sheet for load cell signal conditioners. 
vas: $03-10-201$

Horei ; Dscess oI

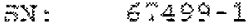

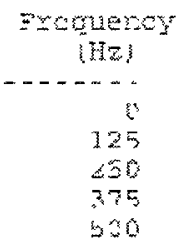

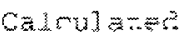

$$
\begin{aligned}
& \text { Chloum } \\
& \text { - } \\
& \text { - } 1.4 \\
& .2 .5 \div 3 \\
& -5.032 \\
& 4750 \\
& +30 \text { पी }
\end{aligned}
$$

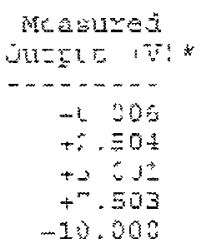

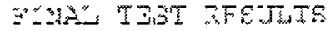

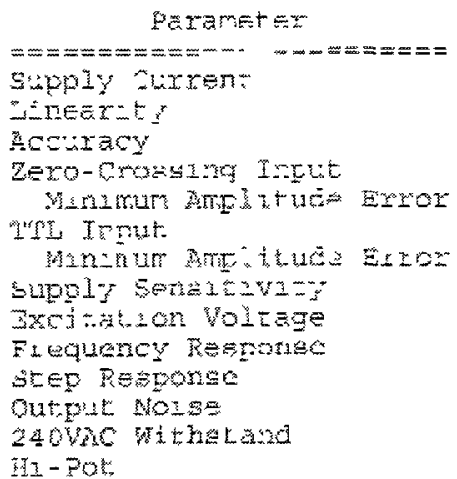

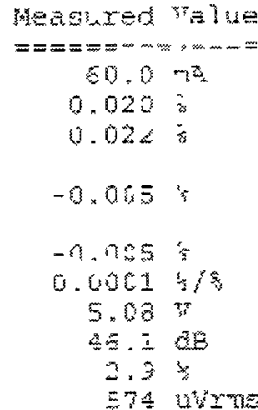

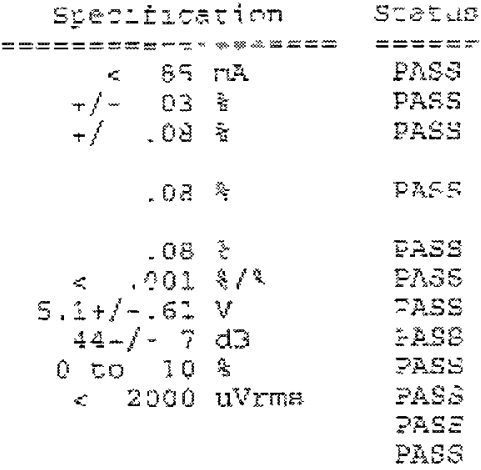

\section{meck Los}

Testerd by:

re:

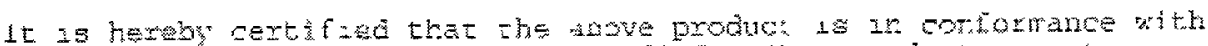

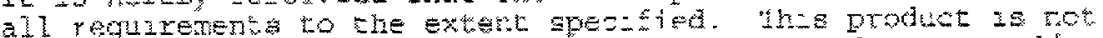

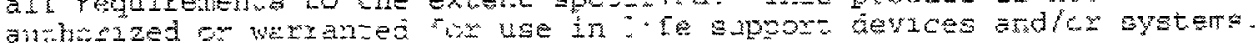

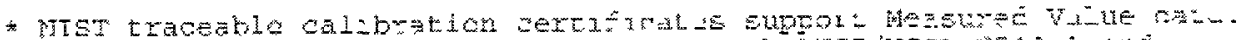

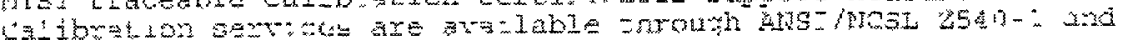

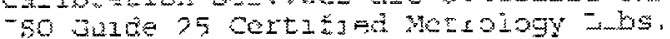

Figure A.4 Factory calibration sheet for RPM frequency signal conditioner. 


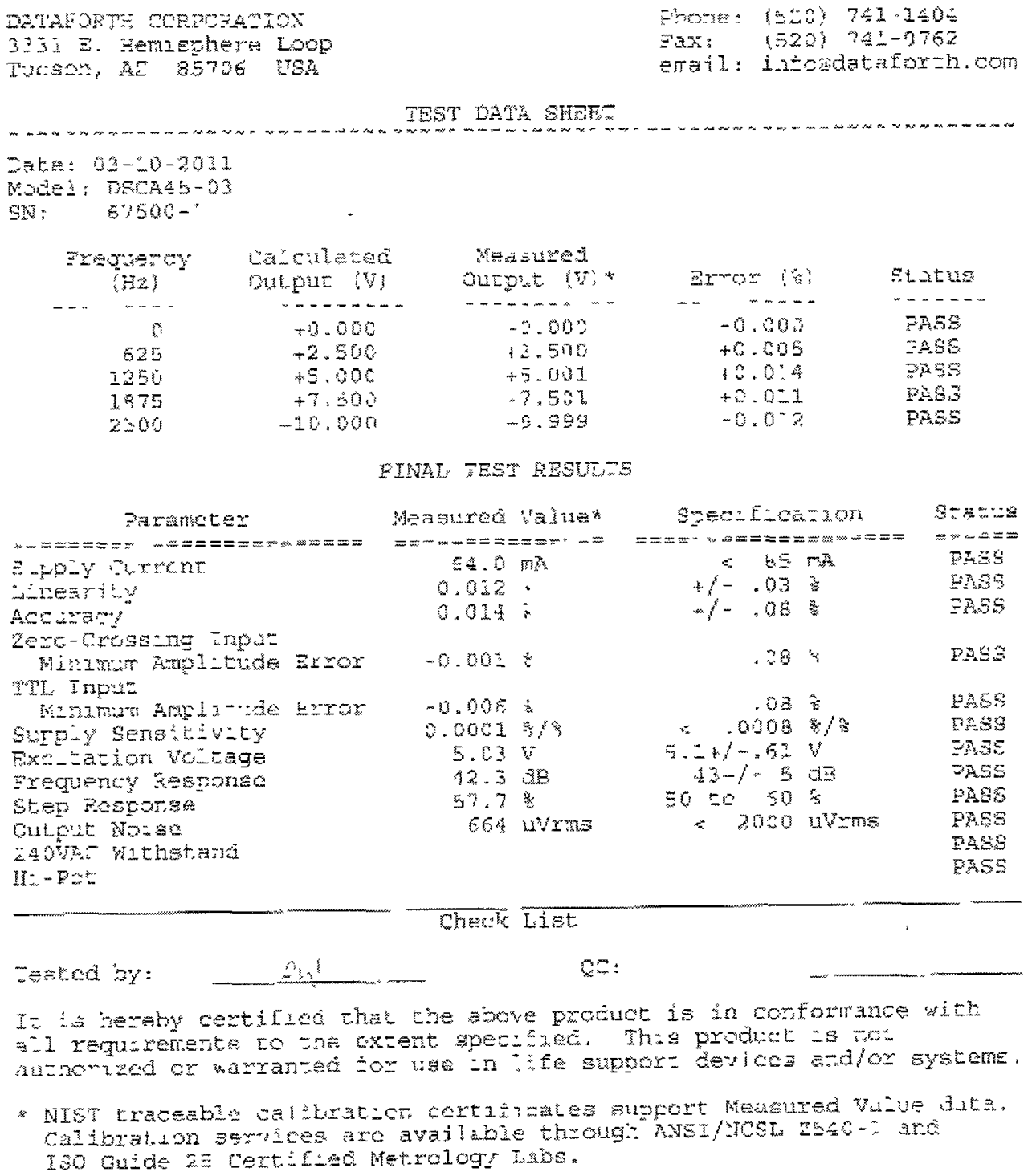

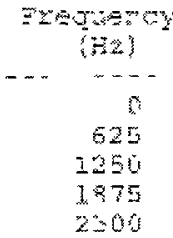

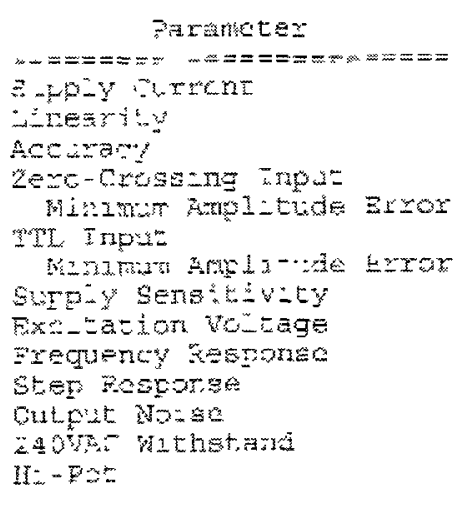

\begin{tabular}{|c|c|}
\hline $\begin{array}{l}\text { Honsured } \\
=\Rightarrow-m-m==0\end{array}$ & 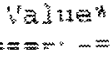 \\
\hline$=4.0$ & $m z$ \\
\hline 0,032 & . \\
\hline 0.012 & $\overline{7}$ \\
\hline$-\infty .00$ & ; \\
\hline-0.035 & 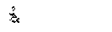 \\
\hline $5.50 \% 1$ & ; \\
\hline 5.03 & 8 \\
\hline 12.3 & 38 \\
\hline $\begin{array}{r}57 \\
66 x\end{array}$ & 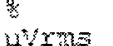 \\
\hline
\end{tabular}

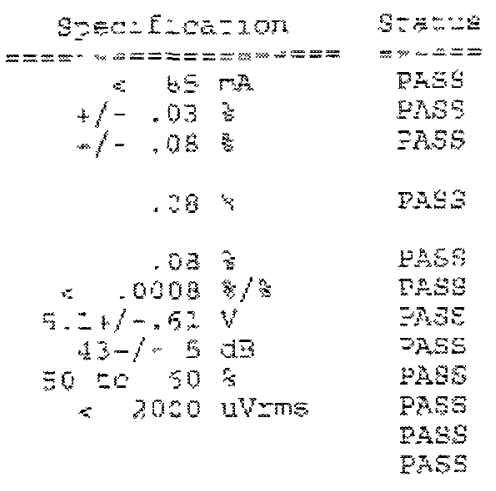

Ghyte List

$-m+\alpha d z$

53

$g i$

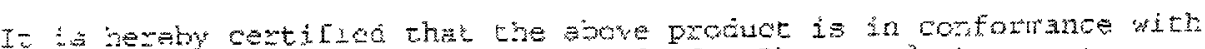

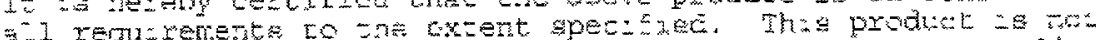

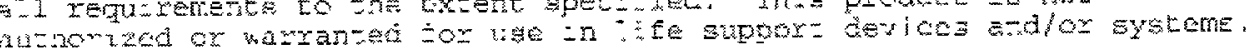

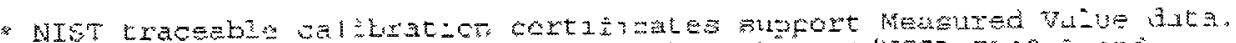

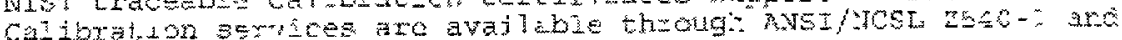

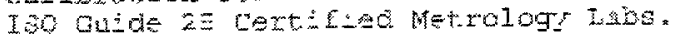

Figure A.5 Factory calibration sheet for carriage speed signal conditioner. 


\section{incerface}

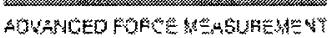

\section{TOROUE TRANSDUCER CALIBRATION CERTIFICATE}

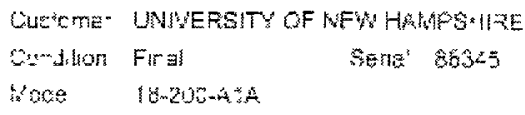

Ca

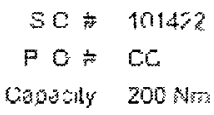

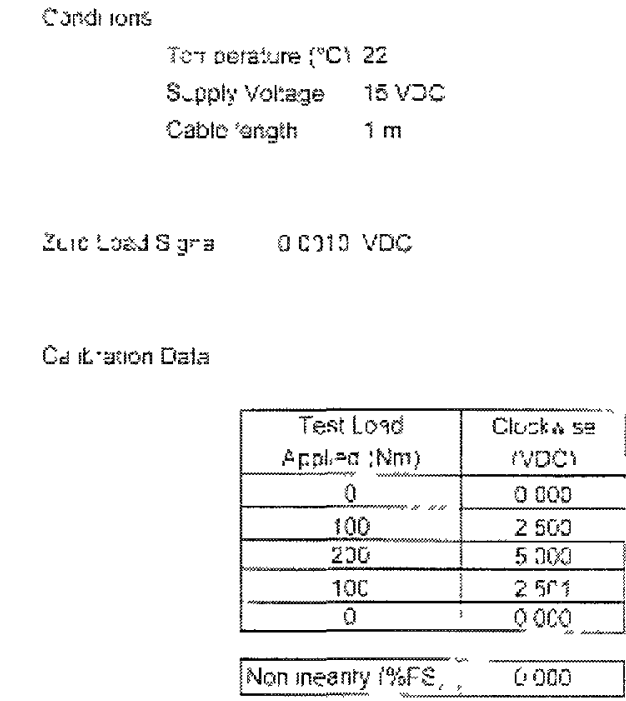

\begin{tabular}{|c|c|}
\hline mirsear & औith \\
\hline 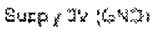 & Mists \\
\hline 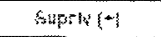 & 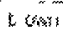 \\
\hline$\Xi 210113$ & sas \\
\hline $5: 1510060403$ & $x+8$ \\
\hline
\end{tabular}

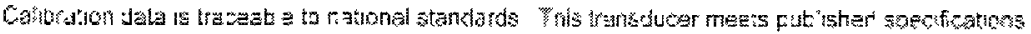

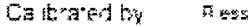
Date
$27 \cdot 3 \pi n \times \frac{1}{1}$

Bnterface lno

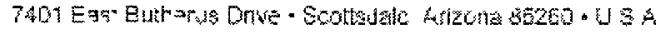

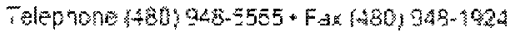

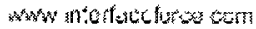

Figure A.6 Factory calibration sheet for torque transducer. 


\section{Appendix B}

\section{B.1 Suggestions for Tow Tank Improvement}

To further develop the hydrokinetic turbine test bed and for other towing requirements, modifications to the tow tank system are recommended. To begin to understand the dynamics of the tow system, a simulation involving a simplified model, depicted in Figure B.1, was performed using MATLAB's ODE45 routine to solve the equation of motion for the system.

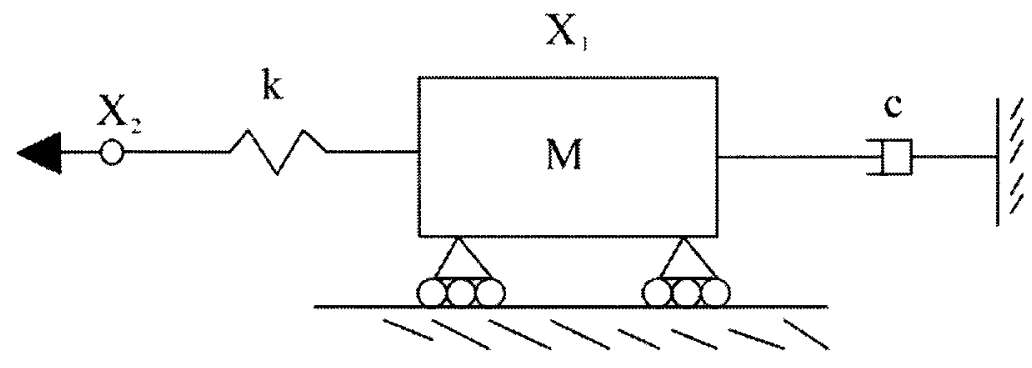

Figure B.1 Simplified model of tow carriage system.

Parameters used:

- $\quad M=168 \mathrm{~kg}$ (Darnell, 1996)

- $a=1 / 6 \mathrm{~m} / \mathrm{s}^{2}$ (estimated from measurements)

- $c=0$

- $k=14000 \mathrm{~N} / \mathrm{m}$ (estimated from cable dimensions and modulus of elasticity of steel) 
Assumptions:

- Cable is accelerating at constant value, $a$.

Governing equations:

$$
M \ddot{X}_{1}+c \dot{X}_{1}+k X_{1}=k X_{2}
$$

Assuming zero initial position and velocity

$$
\begin{gathered}
X_{2}=\frac{1}{2} a t^{2} \\
\ddot{X}_{2}=a
\end{gathered}
$$

Substitute $X_{2}$

$$
M \ddot{X}_{1}+c \dot{X}_{1}+k X_{1}=\frac{1}{2} a t^{2}
$$

Break down into 1st order equations for MATLAB ODE45 solver:

$$
\begin{gathered}
\dot{X}_{1}=Y \\
\dot{Y}=\frac{1}{M}\left(\frac{k}{2} t^{2}-c Y-k X_{1}\right)
\end{gathered}
$$


Figure B.2 shows carriage speed measurements taken using the 60 pulse-perrevolution encoder wheel riding with the carriage, running into a frequency signal conditioner along with the model carriage speed $\left(X_{1}\right)$ with zero initial conditions from 0 to 3 seconds. Sample rate for the measurements is is $500 \mathrm{~Hz}$ and data is filtered with MATLAB's smoothing filter, using a 100 sample wide window. A Nortek-AS Vectrino+ ADV was the only object in the tank producing drag, but it is neglected in the model.

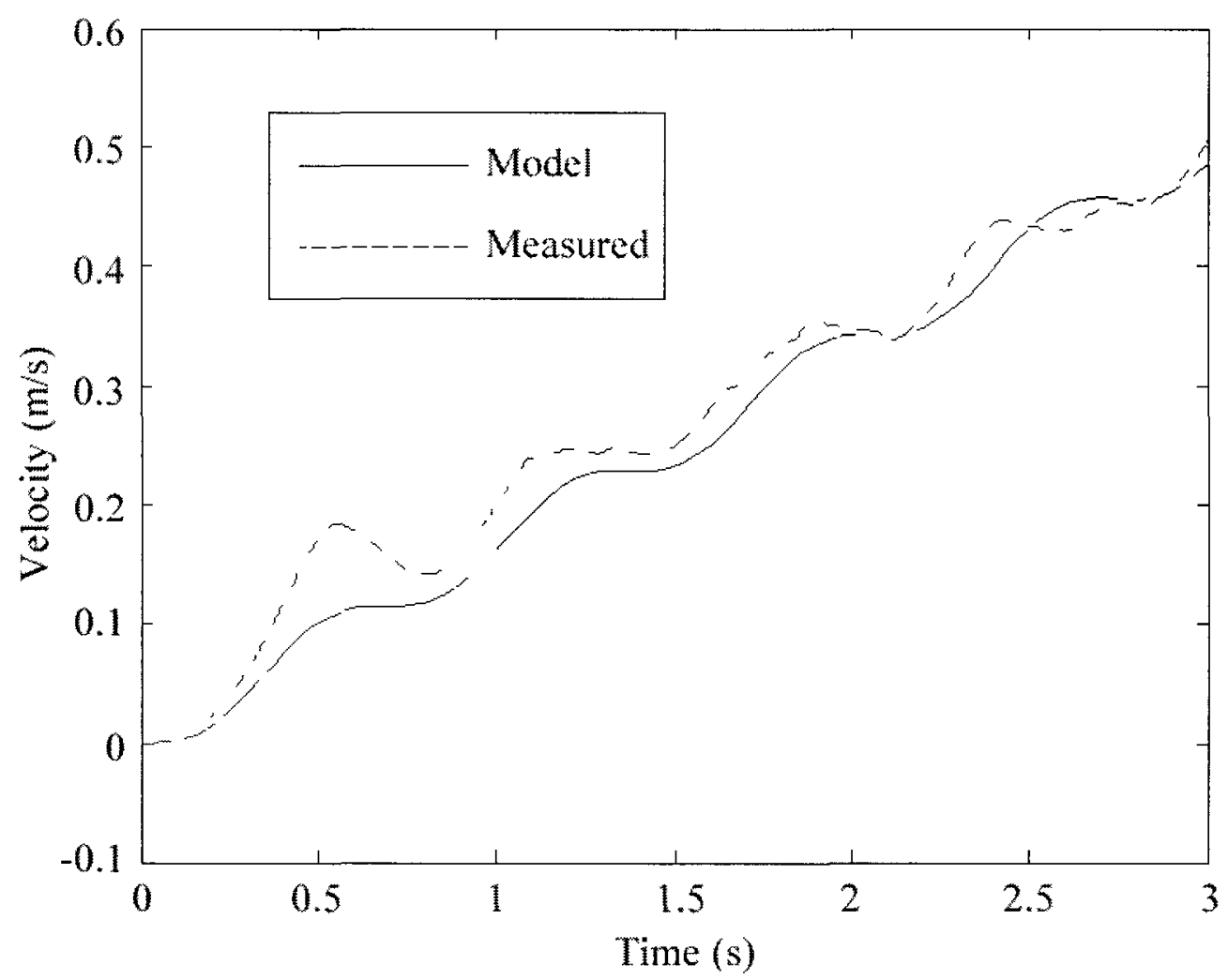

Figure B.2 Tow carriage velocity during acceleration for the model and measurements.

The simulation is quite close to the measurements, showing that the system behaves as if it has negligible damping or friction. Introducing friction could help system stability, but will require more tow power. Adding damping could be as simple as increasing friction at the carriage wheel bearings. The carriage wheels could also be replaced by a 
sort of plain bearing slide type system, but this may introduce wear issues. Positioning accuracy would be improved by stiffer cable, but will oscillate at higher frequencies. This could be improved with more damping, requiring more tow power. It also needs to be taken into account that fluid drag and mass of gear on carriage will change dynamics of system. Some resonance in the tow system was observed during some of the turbine towing experiments that seemed to be excited by the rotating turbine.

Other than the dynamics issues, more tow power is necessary to tow similar frontal area turbines at higher speeds. Also, higher acceleration is necessary to improve steady state operation times. To begin to address these issues, a list of tow tank performance assumptions and target metrics was created:

Assumptions:

- Carriage mass: $1000 \mathrm{lbm}$ (Conservative estimate, $370 \mathrm{lbm}$ from Darnell, 1996)

- Maximum onboard equipment mass (carriage excluded): $500 \mathrm{lbm}$

- Max total mass (carriage plus onboard equipment): $1500 \mathrm{lbm}(680 \mathrm{~kg}$ )

Target metrics:

- Target maximum speed: $3 \mathrm{~m} / \mathrm{s}$

- Maximum steady state drag @ $3 \mathrm{~m} / \mathrm{s}: 425 \mathrm{lbf}$ (corresponds with $0.6 \mathrm{~m}^{2}$ turbine frontal area)

- Maximum steady ideal tow power @ $3 \mathrm{~m} / \mathrm{s}: \sim 5.7 \mathrm{~kW}$ (7.6 hp)

- Max tow speed for $1.3 \mathrm{~m}^{2}$ turbine: $2.0 \mathrm{~m} / \mathrm{s}$

- Target acceleration/deceleration (constant): $1.5 \mathrm{~m} / \mathrm{s}^{2}$

- Maximum transient load with $1500 \mathrm{lbm}$ to $3 \mathrm{~m} / \mathrm{s}$ (drag plus mass times acceleration): $654 \mathrm{lbf}$ 
- Resulting ideal transient tow power to reach $3 \mathrm{~m} / \mathrm{s}: \sim 8.7 \mathrm{~kW}(11.7 \mathrm{hp})$

- Resulting steady state time @ $3 \mathrm{~m} / \mathrm{s}$ (assuming 30m usable length): $8 \mathrm{~s}$

- Resulting steady time @ $1.5 \mathrm{~m} / \mathrm{s}: 19 \mathrm{~s}$

\section{B.2 Suggestions for Hydrokinetic Turbine Test Bed Improvement}

As mentioned earlier, the greatest deficiency of the test bed is the inability to control turbine RPM, and therefore turbine tip speed ratio. It is recommended that a brushless DC servo motor be used to control speed, being used as a generator when load needs to be provided to the turbine. When being used as a generator, a voltage regulator can be used to recharge the batteries onboard the carriage.

Some of the resonance in the tow system was also present in the uppermost crossmember of the main frame assembly during some tows. If this becomes a problem, it is recommend that non submerged struts be added at a 45 degree angle to stiffen the main frame assembly. 